\title{
Loops de código: automorfismos e representações
}

\author{
Rosemary Miguel Pires
}

TESE APRESENTADA

$\mathrm{AO}$

Instituto DE MATEMÁtica e EstatísticA

\author{
$\mathrm{DA}$ \\ Universidade de SÃo Paulo \\ PARA \\ OBTENÇÃO DO TÍTULO \\ $\mathrm{DE}$ \\ Doutor EM CIÊNCIAS
}

Programa: Matemática

Orientador: Prof. Dr. Alexandre Grichkov

São Paulo, maio de 2011 


\section{Loops de código: automorfismos e representações}

Esta versão definitiva da tese contém as correções e alterações sugeridas pela Comissão Julgadora durante a defesa realizada por Rosemary Miguel Pires em 16/5/2011.

Comissão Julgadora:

- Prof. Dr. Alexandre Grichkov (orientador) - IME-USP

- Prof. Dr. Henrique Guzzo Junior - IME-USP

- Prof. Dr. Andrei Zavarnitsine - NSU-Externo

- Profa. Dra. Marinês Guerreiro - UFV-Externo

- Prof. Dr. Alexandr Kornev - Externo 
A meu filho:

Gabriel Ferreira Pires. 


\section{Agradecimentos}

A Deus, que me deu forças, disciplina e perseverança.

Aos meus pais, Aparecida das Dores Pires e João Miguel Pires, aos meus irmãos Cyntia Miguel Pires e Robson Miguel Pires por todo apoio, incentivo e amor.

Ao meu amado filho Gabriel Ferreira Pires que é uma das razões de todo o meu esforço.

Ao meu namorado Bimael Duarte por estar ao meu lado nos momentos possíveis.

Aos meu amigos Rodrigo, Dylene, Débora, Graciele, Tatiane, Pricila, Juliano, Gustavo e Humberto, pela amizade e apoio nos momentos difíceis e alegres.

Ao professor Alexandre Grichkov pela orientação. 


\section{Resumo}

Neste trabalho, estudamos Loops de Código. Para este estudo, introduzimos os loops de código a partir de códigos pares e depois, provamos que loops de código de posto $n$ podem ser caracterizados como imagem homomórfica de certos loops de Moufang livres com $n$ geradores. Além disso, introduzimos o conceito de vetores característicos associados a um loop de código. Com os resultados da teoria estudada, classificamos todos os loops de código de posto 3 e 4, encontramos todos os grupos de automorfismos externos destes loops e, finalmente, determinamos todas as suas respectivas representações básicas.

Palavras-chave: loops de código, códigos pares, vetores característivos, automorfismos externos, representações. 


\begin{abstract}
This work is about code loops. For this study, we introduce the code loops from even codes and then we prove that code loops of rank $n$ can be characterized as a homomorphic image of a certain free Moufang loops with $n$ generators. Moreover, we introduce the concept of characteristic vectors associated with code loops. With the results of this theory, we classify all the code loops of rank 3 and 4, we find all the groups of outer automorphisms of these loops and finally we determine all their basic representations.
\end{abstract}

Keywords: code loops, even codes, characteristic vectors, outer automorphisms, representations. 


\section{Sumário}

$\begin{array}{ll}\text { Introdução } & 1\end{array}$

1 Introdução aos Loops de Código 3

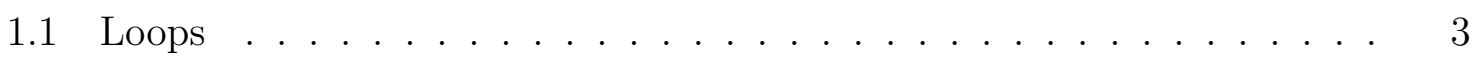

1.2 Loops de Código f . . . . . . . . . . . . . . . . . . . . . . . . . . . . 8

1.3 O loop livre da variedade $\mathcal{E} \ldots \ldots \ldots \ldots$

2 Classificação dos Loops de Código de posto 3 e 4

2.1 Loops de Código de posto $3 \ldots \ldots$. . . . . . . . . . . . . . . 32

2.2 Loops de Código de posto 4 . . . . . . . . . . . . . . . . . 39

2.3 Automorfismos dos Loops de Código de posto 4 . . . . . . . . . 45

3 Representações dos Loops de Código $\quad 63$

3.1 Representações reduzidas dos Loops de Código de posto 3 . . . . . . . . 64

3.2 Representações básicas dos Loops de Código de posto 3 . . . . . . . . 71

3.3 Representações básicas dos Loops de Código de posto 4 . . . . . . . . 75

3.4 Considerações Finais . . . . . . . . . . . . . . . . . . . 80

$\begin{array}{ll}\text { A Tabelas } & 81\end{array}$

$\begin{array}{ll}\text { Referências Bibliográficas } & 92\end{array}$ 


\section{Introdução}

Robert L. Griess (1986) introduziu a teoria de Loops de Código, que tem aplicabilidade para a contrução do grupo "monstro" e de sua álgebra não-associativa (ver [7]). Robert L. Griess demonstrou que um loop de código é um loop de Moufang [1] e Orin Chein e Edgar G. Goodaire (1990) demonstraram que loops de código tem um único comutador não-trivial, um único associador não-trivial e um único quadrado não-trivial. Loops de Moufang, com uma ou mais destas propriedades de "unicidade", têm um papel importante nos trabalhos de alguns autores sobre loops que tem anéis de loop alternativos (ver $[8,9,10])$. Por exemplo, Orin Chein e Edgar G. Goodaire ([11], 1988) provaram que um loop de Moufang, não-associativo com um único quadrado não-trivial, deve ter um anel de loop alternativo sobre qualquer anel de característica dois.

Neste trabalho, estudaremos loops de código e temos como objetivo principal classificar todos os loops de código de posto 3 e 4. Além disso, determinar todos os grupos de automorfismos externos destes loops e suas respectivas representações.

No primeiro capítulo deste trabalho, revisaremos algumas definições e alguns resultados básicos da teoria de loops. Também apresentaremos resultados demonstrados por Chein e Goodaire [3] que serão necessários aos capítulos seguintes. Definiremos códigos pares, e a partir disto, definiremos loops de código. Definiremos E-loops e mostraremos a relação existente entre E-loops e loops de código. Demonstraremos de forma detalhada que um loop de código é um loop de Moufang e provaremos que todo loop de código de posto $n$ é uma imagem homomórfica de um loop de Moufang livre 
com $n$ geradores. No segundo capítulo, faremos um estudo detalhado dos loops de código de posto 3 e 4 . A partir do estudo de vetores característicos associados a um loop de código, demonstraremos que existem 5 loops de código não-isomorfos de posto 3 e 16 loops de código de posto 4, e determinaremos todos os grupos de automorfismos externos destes loops. No terceiro capítulo, definiremos representação de grau $n$ de um loop de código, em particular definiremos representações reduzidas e básicas, e caracterizaremos todas as representações reduzidas básicas para os os loops de código de posto 3 e 4 . 


\section{Capítulo 1}

\section{Introdução aos Loops de Código}

Loops de código foram introduzidos por R.L.Griess Jr. em [1]. Pretendemos neste capítulo dar a noção de códigos pares e loops de código. Com este objetivo revisaremos algumas definições e resultados básicos sobre loops e loops de Moufang.

\section{$1.1 \quad$ Loops}

As definições e resultados iniciais desta seção foram tiradas de [6] e [4].

Definição 1.1.1. Um loop é um conjunto L, munido de uma operação binária (denotada por justaposição ou por · ) tal que, dados quaisquer elementos x, y, z, a equação $x \cdot y=z$ determina o terceiro elemento de modo único e tal que existe um elemento identidade (denotado por 1$)$.

Definição 1.1.2. Um loop $L$ é um loop com a propriedade inversa (denotado por loop P.I.) se cada $x \in L$ tem um único inverso bilateral, que denotamos por $x^{-1}$, e se, para todos $x, y \in L$, o loop satisfaz $x^{-1}(x y)=y$ e $(y x) x^{-1}=y$, ou seja, as propriedades de inverso à esquerda e inverso à direita, respectivamente.

Teorema 1.1.1. Em qualquer loop L, para quaisquer elementos $x, y, z$ de L, as 
seguintes identidades (chamadas identidades de Moufang) são equivalentes:

$$
\begin{aligned}
& ((x y) x) z=x(y(x z)), \\
& ((x y) z) y=x(y(z y)), \\
& (x y)(z x)=(x(y z)) x .
\end{aligned}
$$

Se L é um loop satisfazendo qualquer uma dessas identidades, então L é um loop P.I. que também satisfaz

$$
\begin{gathered}
(y x) x=y x^{2}, \\
x(x y)=x^{2} y, \\
(x y) x=x(y x) .
\end{gathered}
$$

Definição 1.1.3. Um loop L é chamado um loop de Moufang se ele satisfaz qualquer uma das três identidades de Moufang.

Com o objetivo de enunciar um teorema, que é bastante útil para decidirmos se um determinado conjunto não-vazio munido de uma operação binária é um loop, vamos definir a noção de quasigrupo. Seja $G$ um conjunto não-vazio munido de uma operação binária "." (denotado por $(G, \cdot)$ e chamado de grupóide) e seja a qualquer elemento fixado em $G$. As aplicações de translação $L_{a}: G \longrightarrow G$ e $R_{a}: G \longrightarrow G$ são definidas respectivamente por $L_{a}(x)=a \cdot x$ e $R_{a}(x)=x \cdot a$, para cada $x \in G$.

Definição 1.1.4. Um grupóide $(G,$.$) é chamado um quasigrupo se as aplicações$ $L_{a}: G \longrightarrow G$ e $R_{a}: G \longrightarrow G$ são bijeções, para cada $a \in G$.

É importante dizer que a definição de quasigrupo é equivalente a dizermos que dados quaisquer dois elementos de $x, y, z$ em $G$ o terceiro pode ser unicamente selecionado em $G$ de modo que $x \cdot y=z$. Assim, um loop $(L,$.$) é um quasigrupo com elemento$ identidade (bilateral) 1. 
Teorema 1.1.2. Seja $(G,$.$) um grupóide finito. São equivalentes:$

(i) $(G,$.$) é um quasigrupo.$

(ii) $L_{a}: G \longrightarrow G$ e $R_{a}: G \longrightarrow G$ são injetoras, para cada $a \in G$.

(iii) $L_{a}: G \longrightarrow G$ e $R_{a}: G \longrightarrow G$ são sobrejetoras, para cada $a \in G$.

(iv) As leis de cancelamento à esquerda e à direita valem para $(G,$.$) .$

(v) Cada elemento em $G$ aparece somente uma vez em cada linha e em cada coluna da tabela de multiplicação para $(G,$.$) .$

Definição 1.1.5. Sejam $x, y$ e z três elementos de um loop $L$. O comutador de $x$ e $y$ é o único elemento $[x, y]$ de $L$ que satisfaz $x y=(y x)[x, y]$ e o associador de $x, y \mathbf{e}$ $z$ é o único elemento $(x, y, z)$ de L que satisfaz $(x y) z=(x(y z))(x, y, z)$.

Se $X, Y$ e $Z$ são subconjuntos não-vazios de um loop $L$, denotamos por $X^{2},[X, Y]$ e $(X, Y, Z)$, respectivamente, o conjunto de todos os quadrados da forma $x^{2}$, de todos os comutadores da forma $[x, y]$ e de todos os associadores da forma $(x, y, z)$, onde $x \in X$, $y \in Y$ e $z \in Z$.

Definição 1.1.6. Seja $L$ um loop. O núcleo à esquerda de $L$ é o conjunto

$$
\mathcal{N}_{l}=\{a \in L \mid(a, x, y)=1, \text { para todos } x, y \in L\},
$$

$o$ núcleo à direita de $L$ é o conjunto

$$
\mathcal{N}_{r}=\{a \in L \mid(x, y, a)=1, \text { para todos } x, y \in L\},
$$

$o$ núcleo do meio de $L$ é

$$
\mathcal{N}_{m}=\{a \in L \mid(x, a, y)=1, \text { para todos } x, y \in L\}
$$


e o núcleo de $L$ é

$$
\mathcal{N}(L)=\mathcal{N}_{l} \cap \mathcal{N}_{r} \cap \mathcal{N}_{m}
$$

$O$ centro de $L e ́$

$$
\mathcal{Z}(L)=\{x \in \mathcal{N}(L) \mid[a, x]=1, \text { para todo } a \in L\}
$$

Proposição 1.1.1. Todo núcleo de um loop é um subloop associativo e, portanto, um grupo. O centro de um loop é um grupo abeliano.

As demonstrações do Lema 1.1.1, Corolário 1.1.1 e dos Teoremas 1.1.3, 1.1.4 e 1.1.6 a seguir podem ser encontradas no artigo [3].

Lema 1.1.1. Se L é um loop de Moufang, $z \in L$ um elemento que comuta com todo elemento de $L$ e $z^{2} \in \mathcal{N}(L)$, então $z \in \mathcal{Z}(L)$.

Corolário 1.1.1. Se L é um loop de Moufang, $z \in L$ um elemento que comuta com todo elemento de $L$ e $z^{2}=1$, então $z \in \mathcal{Z}(L)$.

Teorema 1.1.3. Se L é um loop de Moufang com um único quadrado não trivial e, então $e^{2}=1$ e L é um grupo abeliano ou $[L, L]=(L, L, L)=L^{2}=\{1, e\} \subseteq \mathcal{Z}(L)$.

Teorema 1.1.4. Se L é um loop de Moufang com $L^{2}=\{1, e\}$, onde e é o único quadrado não trivial de $L$, então, para todo $w, x, y, z \in L$,

$$
\begin{aligned}
{[x y, z] } & =[x, z][y, z](x, y, z) \\
(w x, y, z) & =(w, y, z)(x, y, z) .
\end{aligned}
$$

Teorema 1.1.5. (Moufang) Se a,b,c são elementos de um loop de Moufang e ab.c = $a . b c$, então o subloop gerado por $\{a, b, c\}$ é um grupo.

Para a demonstração do Teorema de Moufang ver Bruck [2]. Por este teorema, se $(x, y, z) \neq 1$, então $\left(x^{\prime}, y^{\prime}, z^{\prime}\right) \neq 1$ para qualquer permutação $x^{\prime}, y^{\prime}, z^{\prime}$ de $x, y, z$. Por- 
tanto, se $L$ tem um único quadrado não trivial e desta forma, um único associador não trivial, então $\left(x^{\prime}, y^{\prime}, z^{\prime}\right)=(x, y, z)$. Em outras palavras, o associador de três elementos é independente da ordem de seus elementos. Assim, a propriedade 1.5 também é válida para $(x, w y, z)$ e $(x, y, w z)$.

Teorema 1.1.6. Suponha que $L$ e $M$ são loops de Moufang com $L^{2}=\{1, e\}$ e $M^{2}=\{1, f\}$, onde e e $f$ são os únicos quadrados não triviais de $L$ e $M$, respectivamente, e suponha que $\left\{x_{1}, \ldots, x_{n}\right\}$ e $\left\{y_{1}, \ldots, y_{n}\right\}$ são conjuntos geradores minimais para $L$ e $M$, respectivamente, com $x_{1}^{2}=$ e e $y_{1}^{2}=f$. Finalmente, suponha que, para todo $1 \neq i, j, k \neq n$, as seguintes condições são válidas:

i) $\left|x_{i}\right|=\left|y_{i}\right|$

ii) $\left[x_{i}, x_{j}\right]=1 \Leftrightarrow\left[y_{i}, y_{j}\right]=1$

iii) $\left(x_{i}, x_{j}, x_{k}\right)=1 \Leftrightarrow\left(y_{i}, y_{j}, y_{k}\right)=1$.

Então $L \simeq M$.

A próxima proposição contém resultados importantes que serão utilizados ao longo do texto. As demonstrações saem diretamente de ([3], Teorema 2).

Proposição 1.1.2. Seja F um loop de Moufang.

1. Se $(x, y, z)^{2}=1$ e todos os comutadores e associadores de $F$ são centrais, então

$$
[x y, z]=[x, z][y, z](x, y, z)
$$

2. Se os comutadores e associadores de F são centrais, então

$$
(w x, y, z)=(w, y, z)(x, y, z)
$$


Usando a proposição anterior se prova que $(x, w y, z)=(x, w, z)(x, y, z)$ e $(x, y, w z)=$ $(x, y, w)(x, y, z)$. De fato, observe que $[x y, z]=[z, x y]$. Daí, $(x, y, z)=(z, x, y)$. $\operatorname{Logo}(x, y, w z)=(w z, x, y)=(w, x, y)(z, x, y)=(y, w, x)(y, z, x)=(x, y, w)(x, y, z)$. Analogamente temos o outro caso.

Proposição 1.1.3. Se os quadrados e comutadores de um loop de Moufang $F$ são centrais, então

$$
(x y)^{2}=x^{2} y^{2}[x, y]
$$

Demonstração. Temos $(x y)^{2}=(x y)((x y)[x, y])$. Como $[x, y] \in \mathcal{Z}(F)$, obtemos $(x y)^{2}=(x y)(y x) \cdot[x, y]$. Usando a identidade de Moufang $x y \cdot z x=(x \cdot y z) x$ ficamos com $(x y)^{2}=\left(x y^{2}\right) x .[x, y]$. Como $y^{2} \in \mathcal{Z}(F)$, obtemos a igualdade desejada.

\subsection{Loops de Código}

O objetivo desta seção é definir loops de código, exemplificar e mostrar a existência de loops de código. Primeiramente definiremos e exemplificaremos o que é um código par, mostrando alguns resultados relevantes sobre este. Para isto, consideremos $\mathbf{F}_{2}^{n}$ como um espaço vetorial $n$-dimensional sobre o corpo de 2 elementos $\mathbf{F}_{2}=\{0,1\}$. Para vetores $u$ e $v$ em $\mathbf{F}_{2}^{n},|v|$ (o peso de $v$ ) denota o número de coordenadas não-nulas de $v$ e $|u \cap v|$ (peso de $u$ intersecção $v$ ) denota o número de posições nas quais as coordenadas de $u$ e $v$ são ambas não-nulas, ou seja, se $u=\left(u_{1}, \ldots, u_{n}\right)$ e $v=\left(v_{1}, \ldots, v_{n}\right)$, com $u_{i}, v_{i} \in \mathbf{F}_{2}$, para todos $i=1, \ldots, n$, então

$$
|v|=\left|\left\{i \mid v_{i}=1\right\}\right| \text { e }|u \cap v|=\left|\left\{i \mid u_{i}=v_{i}=1\right\}\right|
$$

Definição 1.2.1. Um código par é um subespaço $V \subseteq \mathbf{F}_{2}^{n}$ tal que $|v| \equiv 0(\bmod 4) e$ $|u \cap v| \equiv 0(\bmod 2)$, para quaisquer $u, v \in V$. 
Exemplo 1.2.1. Seja o subespaço $V \subseteq \mathbf{F}_{2}^{7}$ com base $B=\left\{v_{1}, v_{2}, v_{3}\right\}$ onde

$$
v_{1}=(1,1,1,1,0,0,0), \quad v_{2}=(1,1,0,0,1,1,0), \quad v_{3}=(1,0,1,0,1,0,1) .
$$

Então $V=\left\{v_{0}=0, v_{1}, v_{2}, v_{3}, v_{4}=v_{1}+v_{2}, v_{5}=v_{1}+v_{3}, v_{6}=v_{2}+v_{3}, v_{7}=v_{1}+v_{2}+v_{3}\right\}$ com

$$
\begin{aligned}
& v_{4}=(0,0,1,1,1,1,0), \quad v_{5}=(0,1,0,1,1,0,1), \\
& v_{6}=(0,1,1,0,0,1,1), \quad v_{7}=(1,0,0,1,0,1,1) .
\end{aligned}
$$

Temos que $V$ é um código par, pois $\left|v_{i}\right|=4, i=1, \ldots, 7, \operatorname{logo},|v| \equiv 0 \bmod 4$, para todo $v \in V$ e temos que $\left|v_{i} \cap v_{j}\right| \equiv 0 \bmod 2$, para todos $v_{i}, v_{j} \in V, i, j=0, \ldots, 7$. De fato, $\left|v_{i} \cap v_{i}\right|=4, i=1, \ldots, 7,\left|v_{i} \cap v_{j}\right|=2, i, j=1, \ldots, 7, i \neq j e\left|v_{0} \cap v_{i}\right|=0, i=0,1, \ldots, 7$.

Seja $V$ um código par. Denotemos por $L(V)$ o conjunto $\{1,-1\} \times V$.

Definição 1.2.2. Seja a função (chamada fator de conjunto) $\phi: V \times V \rightarrow\{1,-1\}$, definida para todo $u, v, w \in V$, por :

1. $\phi(v, v)=(-1)^{\frac{|v|}{4}}$;

2. $\phi(v, w)=(-1)^{\frac{|v \cap w|}{2}} \phi(w, v)$;

3. $\phi(0, v)=\phi(v, 0)=1$;

4. $\phi(v+w, u)=\phi(v, w+u) \phi(v, w) \phi(w, u)(-1)^{|v \cap w \cap u|}$, onde $|v \cap w \cap u| \operatorname{denota} o$ número de posições nas quais as coordenadas de $u, v$ e $w$ são todas não-nulas.

A existência da função fator de conjunto é mostrada por Robert L. Griess em [1]. Vamos definir uma operação binária “." sobre $L(V)$, chamada de produto e deno- 
tada, quando conveniente, por justaposição. Sejam $v, w \in V$, definimos

$$
\begin{aligned}
& v \cdot w=\phi(v, w)(v+w), \text { onde } \phi(v, w) \in\{1,-1\}, \\
& v \cdot(-w)=(-v) \cdot w=-(v \cdot w), \\
& (-v) \cdot(-w)=v \cdot w .
\end{aligned}
$$

Como $L(V)$ é definido como o produto cartesiano de $\{1,-1\}$ por $V$, na definição acima identificamos $v$ com $(1, v)$ e $-v \operatorname{com}(-1, v)$. De maneira geral, denotamos um elemento de $L(V)$ da forma $x=a v$, onde $a \in\{1,-1\}$ e $v \in V$. Assim, dois elementos $x, y$ de $L(V)$ da forma $x=a u$ e $y=b v$, com $a, b \in\{1,-1\}$, são iguais se $a=b$ e $u=v$. É bom observar também que podemos representar o produto de dois elementos quaisquer $x=a u$ e $y=b v$ de $L(V)$ da forma $x y=(a b \phi(u, v))(u+v)$.

Proposição 1.2.1. Com o produto definido acima, $(L(V), \cdot)$, ou simplesmente $L(V)$, tem uma estrutura de loop.

Demonstração. Como $L(V)$ é finito, basta provarmos que, para todo $a \in L(V)$, as aplicações de translação $L_{a}: G \longrightarrow G$ e $R_{a}: G \longrightarrow G$, definidas respectivamente por $L_{a}(x)=a \cdot x$ e $R_{a}(x)=x \cdot a$, para todo $x \in G$ são injetoras ( pelo Teorema 1.1.2) $\mathrm{e}$ que $L(V)$ tem elemento identidade.

Com efeito, sejam $x=a u \in L(V)$ e as aplicações de translação $L_{x}: G \longrightarrow G$ e $R_{x}: G \longrightarrow G$ definidas como antes. Sejam $y=b v$ e $z=c w$ elementos de $L(V)$ tais que $L_{x}(y)=L_{x}(z)$. Pela definição de $L_{x}$ obtemos $x y=x z$, ou seja, $(a u)(b v)=(a u)(c w)$ e pela definição do produto em $L(V)$ temos $(a b \phi(u, v))(u+v)=(a c \phi(u, w))(u+w)$. Pela igualdade, obtemos $a b \phi(u, v)=a c \phi(u, w)$ e $u+v=u+w$, o que nos dá $v=w$ e $\phi(u, v)=\phi(u, w)$ e finalmente, $b=c$. Logo $y=b v=c w=z$, como queríamos. Portanto, $L_{x}$ é injetora, para todo $x \in L(V)$. Analogamente, prova-se que $R_{x}$ é injetora, para todo $x \in L(V)$. Agora, 0 é o elemento identidade de $L(V)$, pois $0 \cdot v=\phi(0, v)(0+v)=1 v=v$ e $0 \cdot(-v)=-0 \cdot v=-1 v=-v$. 
O lema abaixo estabelece algumas propriedades relativas ao código par $V$, que podem ser encontradas no artigo de Griess [1].

Lema 1.2.1. Sejam $x, y, z$ quaisquer elementos de um código par $V$. Então:

a) $|x \cap y \cap(z+x)|=|x \cap y \cap z|$;

b) $\frac{1}{2}|x \cap(x+y+z)|=\frac{1}{2}|x \cap y|+\frac{1}{2}|x \cap z|+|x \cap y \cap z|$;

c) $\frac{1}{2}|y \cap(x+z)|=\frac{1}{2}|y \cap x|+\frac{1}{2}|y \cap z|+|y \cap x \cap z|$.

Robert L. Griess Jr. demonstrou em [1] que um loop de código é um loop de Moufang e, no artigo [3], Orin Chein e Edgar G. Goodaire demonstraram que um loop de código satisfaz as propriedades do próximo teorema. Com base nas idéias destes artigos demonstraremos o próximo teorema.

Teorema 1.2.1. Um loop $L(V)$ é um loop de Moufang e valem as seguintes propriedades, para quaisquer $u, v, w \in V$ :

a) $v^{2}=(-1)^{\frac{|v|}{4}} 0$,

b) $[u, v]=u^{-1} v^{-1} u v=(-1)^{\frac{|u \cap v|}{2}} 0$,

c) $(u, v, w)=((u v) w)\left((u(v w))^{-1}\right)=(-1)^{|u \cap v \cap w|} 0$.

Demonstração. Seja $L(V)=\{1,-1\} \times V$, onde $V$ é um código par. Provemos que $L(V)$ satisfaz uma das identidades de Moufang, a saber,

$$
(a x)(b y) \cdot(c z)(a x)=[(a x) \cdot(b y)(c z)](a x), \text { para todos } a x, b y, c z \in L(V) .
$$

Pela definição do produto em $L(V)$ para obtermos (1.3) basta provarmos

$$
\phi(x, y) \phi(z, x) \phi(x+y, z+x)=\phi(y, z) \phi(x, y+z) \phi(x+y+z, x)
$$


onde $\phi: V \times V \rightarrow\{1,-1\}$ é a função fator de conjunto.

De fato, por um lado,

$$
(a x)(b y) \cdot(c z)(a x)=(\operatorname{abac} \phi(x, y) \phi(z, x) \phi(x+y, z+x))(y+z) .
$$

Por outro lado,

$$
[(a x) .(b y)(c z)](a x)=(a b a c \phi(y, z) \phi(x, y+z) \phi(x+y+z, x))(y+z) .
$$

Pela Definição 1.2.2 a função fator de conjunto $\phi: V \times V \rightarrow\{1,-1\}$ satisfaz as seguintes propriedades:

$$
\begin{aligned}
\phi(v, w) & =(-1)^{\frac{v \cap w}{2}} \phi(w, v) \\
\phi(v+w, u) & =\phi(v, w+u) \phi(v, w) \phi(w, u)(-1)^{|v \cap w \cap u|} .
\end{aligned}
$$

Pelo Lema 1.2.1 e pela equação (1.6),

$$
\begin{aligned}
\phi(x, y) \phi(x+y, z+x) & =\phi(y, z+x) \phi(x, x+y+z)(-1)^{|x \cap y \cap z|}= \\
& =\phi(y, z+x) \phi(x+y+z, x)(-1)^{\frac{|x \cap y|}{2}+\frac{|x \cap z|}{2}} .
\end{aligned}
$$

Em (1.5) ficamos com

$$
\phi(y, z+x) \phi(x+y+z, x) \phi(z, x)(-1)^{\frac{|x \cap y|}{2}+\frac{|x \cap z|}{2}}=\phi(y, z) \phi(x, y+z) \phi(x+y+z, x) .
$$

Logo a equação (1.5) é equivalente à:

$$
\phi(z, x) \phi(y, z) \phi(x, y+z) \phi(y, z+x)(-1)^{\frac{|x \cap y|}{2}+\frac{|x \cap z|}{2}}=1 .
$$


Mas $\phi(z, x)=(-1)^{\frac{|x \cap z|}{2}} \phi(x, z)$, por (1.5). Assim, (1.4) é equivalente à

$$
\phi(x, z) \phi(y, z) \phi(x, y+z) \phi(y, z+x)(-1)^{\frac{|x \cap y|}{2}}=1 .
$$

Agora para provarmos a equação (1.7) basta observarmos que, pelo Lema 1.2.1c) e pelas equações (1.5) e (1.6), temos:

$$
\begin{aligned}
\phi(x, z) \phi(x, z+y) & =\phi(x+z, y) \phi(z, y)(-1)^{|x \cap y \cap z|} ; \\
\phi(y, x+z) & =\phi(x+z, y)(-1)^{\frac{y \cap x}{2}+\frac{y \cap z}{2}+|y \cap x \cap z|} ; \\
\phi(y, z) & =(-1)^{\frac{|y \cap z|}{2}} \phi(z, y) .
\end{aligned}
$$

Portanto, $L(V)$ é um loop de Moufang.

Agora provaremos as propriedades mencionadas no Teorema. Como usamos a identificação av para um elemento de $L(V)$, com $a \in\{1,-1\}$ e $v \in V$, notemos que -0 corresponde a $(-1,0) \in L(V)$, onde 0 é o elemento identidade de $V$.

a) Como $v^{2}=\phi(v, v)(v+v)$ e, por definição, $\phi(v, v)=(-1)^{\frac{|v|}{4}}, \operatorname{logo} v^{2}=(-1)^{\frac{|v|}{4}} 0$.

b) Por definição,

$$
\begin{aligned}
u \cdot v & =\phi(u, v)(u+v)=(-1)^{\frac{|u \cap v|}{2}} \phi(v, u)(v+u)= \\
& =(-1)^{\frac{|u \cap v|}{2}} 0 \cdot \phi(v, u)(v+u)=(-1)^{\frac{|u \cap v|}{2}} 0 \cdot(v \cdot u) .
\end{aligned}
$$

Portanto, pela unicidade do comutador, temos $[u, v]=(-1)^{\frac{|u \cap v|}{2}} 0$.

c) Por definição, por um lado,

$$
\begin{aligned}
(u v) w & =\phi(u, v) \phi(u+v, w)(u+v+w)= \\
& =(-1)^{|u \cap v \cap w|} \phi(v, w) \phi(u, v+w)(u+v+w) .
\end{aligned}
$$


Por outro lado,

$$
\begin{aligned}
(-1)^{|u \cap v \cap w|} 0 \cdot(u(v w)) & =(-1)^{|u \cap v \cap w|} 0 \cdot(\phi(v, w) \phi(u, v+w)(u+v+w))= \\
& =(-1)^{|u \cap v \cap w|} \phi(v, w) \phi(u, v+w) \phi(0, u+v+w)(u+v+w)= \\
& =(u v) w .
\end{aligned}
$$

Portanto, pela unicidade do associador, temos $(u, v, w)=(-1)^{|u \cap v \cap w|} 0$.

Definição 1.2.3. O loop de Moufang $L(V)$ é chamado de loop de código. Dizemos que $L(V)$ tem posto $m$, se $\operatorname{dim}_{\mathbf{F}_{2}} V=m$.

Exemplo 1.2.2. Seja $V$ o código par do exemplo 1.2.1 e seja $L(V)=\{1,-1\} \times V$, com a operação de multiplicação definida como acima. Logo $|L(V)|=16$. Vamos encontrar a tabela de multiplicação de $L(V)$ (1.1) usando o fato de que $L(V)$ é um loop de Moufang e as propriedades provadas anteriormente. Identificaremos $v_{i}$ com $i$ $e-v_{i}$ com $-i$. Aqui, $-(-v)$ significa $(-0)(-v)$, então $-(-v)=v$.

\begin{tabular}{|l||r|r|r|r|r|r|r|r|r|r|r|r|r|r|r|r|}
\hline & 0 & 1 & 2 & 3 & 4 & 5 & 6 & 7 & -0 & -1 & -2 & -3 & -4 & -5 & -6 & -7 \\
\hline \hline 0 & 0 & 1 & 2 & 3 & 4 & 5 & 6 & 7 & -0 & -1 & -2 & -3 & -4 & -5 & -6 & -7 \\
\hline 1 & 1 & -0 & 4 & 5 & -2 & -3 & 7 & -6 & -1 & 0 & -4 & -5 & 2 & 3 & -7 & 6 \\
\hline 2 & 2 & -4 & -0 & 6 & 1 & -7 & -3 & -5 & -2 & 4 & 0 & -6 & -1 & 7 & 3 & 5 \\
\hline 3 & 3 & -5 & -6 & -0 & -7 & 1 & 2 & -4 & -3 & 5 & 6 & 0 & 7 & -1 & -2 & 4 \\
\hline 4 & 4 & 2 & -1 & 7 & -0 & -6 & 5 & -3 & 4 & 2 & -1 & 7 & -0 & 6 & 5 & -3 \\
\hline 5 & 5 & 3 & 7 & -1 & 6 & -0 & -4 & -2 & 5 & 3 & -7 & -1 & -6 & -0 & -4 & -2 \\
\hline 6 & 6 & -7 & 3 & -2 & -5 & 4 & -0 & 1 & 6 & -7 & 3 & -2 & -5 & 4 & -0 & 1 \\
\hline 7 & 7 & 6 & 5 & 4 & 3 & 2 & -1 & -0 & 7 & 6 & 5 & 4 & 3 & 2 & -1 & -0 \\
\hline
\end{tabular}

Tabela 1.1: Tabela de Multiplicação de $L(V)$ referente ao Exemplo 1.2.2.

Assim, $L(V)$ é um loop de código de posto 3.

Definição 1.2.4. Um loop de Moufang L é chamado E-loop se existe um subloop central $Z$ com 2 elementos tal que $L / Z \in A$, onde $A$ é a variedade de grupos com identidade $x^{2}=1$. 
Exemplo 1.2.3. $\mathbb{Z}_{2}, \mathbb{Z}_{4}$ e $Q_{8}$.

O resultado principal desta seção relaciona loops de código e E-loops. Para demonstrarmos este resultado precisamos do próximo lema e do teorema principal demonstrado por Chein e Goodaire em [3].

Lema 1.2.2. Seja L um loop de Moufang finito. O loop L é um E-loop se, e somente se, $\left|L^{2}\right| \leq 2$.

Demonstração. Seja $L$ um E-loop. Então existe um subloop central $Z=\{1, x\}$ tal que $\bar{y}^{2}=\overline{1}, \forall \bar{y} \in L / Z$. Vamos mostrar que $L^{2} \subseteq Z$. Se $e \in L^{2}$ é tal que $e \neq 1, x$, então existirá $u \in L$ tal que $u^{2}=e$. Daí teremos $\bar{u}^{2} \neq 1$, contradizendo a definição de $L$. Logo $u^{2}=1$ ou $u^{2}=x$, ou seja $L^{2} \subseteq Z$. Portanto, $\left|L^{2}\right| \leq 2$.

Reciprocamente, suponhamos $\left|L^{2}\right| \leq 2$. Caso $\left|L^{2}\right|=1$, então $L$ é um loop de Moufang comutativo. De fato, para quaisquer $x, y \in L,[x, y]=x^{-2}\left(x y^{-1}\right)^{2} y^{2}=1$. Logo, pelo Corolário 1.1.1, qualquer elemento de $L$ está no centro de $L$. Considere então um subloop central qualquer com dois elementos, digamos $Z(L)=\{1, x\}$, onde $x \in L$ é qualquer. Assim, $L / Z(L) \in A$, onde $A$ é a variedade de grupos tal que $y^{2}=1$, para todo $y \in L / Z(L)$. Agora suponhamos $L^{2}=\{1, e\}=Z$. Pelo Teorema 1.1.3, $L^{2}$ é um subloop central e assim podemos ver com simples cálculos que $\bar{y}^{2}=\overline{1}$, para todo $\bar{y} \in L / Z$.

Teorema 1.2.2. (Chein e Goodaire, [3]) Um loop finito L é isomorfo a um loop de código se, e somente se, $L$ é um loop de Moufang com $\left|L^{2}\right| \leq 2$.

Teorema 1.2.3. Um loop de Moufang L é um loop de código se, e somente se, L é um E-loop finito.

Demonstração. A demonstração sai diretamente do Lema 1.2.2 e do Teorema 1.2.2.

A seguir introduzimos alguns loops de Moufang $\mathcal{F}_{n}$ e provamos que todo $E$-loop é uma imagem homomórfica de $\mathcal{F}_{n}$. Depois classificaremos os $E$-loops de postos 3 e 4 . 
Definição 1.2.5. Seja $\mathcal{E}$ a variedade de loops de Moufang com as seguintes identidades:

$$
\begin{array}{lll}
x^{4}=1, & {[x, y]^{2}=1, \quad} & (x, y, z)^{2}=1, \\
{\left[x^{2}, y\right]=1,} & {[[x, y], t]=1,} & {[(x, y, z), t]=1,} \\
\left(x^{2}, y, z\right)=1, & ([x, y], z, t)=1, & ((x, y, z), t, s)=1 .
\end{array}
$$

Observemos que, pelo Teorema 1.2.1, um loop de código é Moufang e possui um único quadrado não trivial. Com isto e pelo Teorema 1.1.3, todos os E-loops estão contidos em $\mathcal{E}$.

\subsection{O loop livre da variedade $\mathcal{E}$}

Seja $V$ um $\mathbf{F}_{2}$-espaço com uma base $\left\{v_{1}, v_{2}, \ldots\right\}$. Identificaremos cada elemento de $V$ com um subconjunto finito do conjunto dos números naturais $\mathbb{N}$ do seguinte modo: Consideramos a correspondência biunívoca $V \longrightarrow \mathcal{P}(\mathbb{N})$, onde $v=a_{1} v_{1}+a_{2} v_{2}+\cdots \in$ $V \longmapsto \sigma(v)=\sigma=\left\{i \mid a_{i}=1\right\} \in \mathcal{P}(\mathbb{N})$. Deste modo, usaremos a notação $\sigma \in V$ quando $\sigma=\sigma(v)$, para $v \in V$. Além disso, observamos que, $\sigma(v+w)=\sigma(v) \Delta \sigma(w)$, onde $\sigma \Delta \mu=(\sigma \backslash \mu) \cup(\mu \backslash \sigma)$.

Sejam $W=V \wedge V$ e $U=V \wedge V \wedge V$ produtos anti-simétricos de $V$. Isto significa que $W$ tem uma base $\{i \wedge j \mid i \wedge j=j \wedge i, i \wedge i=0 ; i, j \in \mathbb{N}\}$ e $U$ tem uma base $\{i \wedge j \wedge k \mid i \wedge j \wedge k=j \wedge k \wedge i=j \wedge i \wedge k ; i, j, k \in \mathbb{N}\}$. Além disso, $i \wedge j \wedge k=0$ se, e somente se, $|\{i, j, k\}|<3$. Seja $\bar{V}$ uma cópia isomórfica de $V$ com uma base $\{\bar{i} \mid i \in \mathbb{N}\}$.

Para o que segue, dado um elemento $v \in V$, denotaremos seu correspondente $\sigma=\sigma(v)$ por $\sigma=\left\{i_{1}, i_{2}, \ldots, i_{s}\right\}$, com $i_{1}<i_{2}<\cdots<i_{s}$. Deste modo, escrevere$\left.\left.\operatorname{mos} \sigma=\left(i_{1} i_{2}\right) i_{3}\right) \ldots\right) i_{s}$.

Considere o conjunto $\mathcal{F}$ definido pelo produto cartesiano dos $\mathbf{F}_{2}$-espaços $V, \bar{V}, W$ e $U$, ou seja, $\mathcal{F}=V \cdot \bar{V} . W . U$. Queremos dar uma estrutura de loop de Moufang para $\mathcal{F}$. 
Definimos

$$
\begin{gathered}
i^{2}=\bar{i},[i, j]=i \wedge j,(i, j, k)=i \wedge j \wedge k, i, j, k \in \mathbb{N} \\
\sigma . \mu=\sigma \Delta \mu \cdot \prod_{i \in \sigma \cap \mu} \bar{i} \cdot \prod_{i \in \sigma, j \in \mu, i>j} i \wedge j \cdot \prod_{i \in \sigma, j, k \in \mu, j<k} i \wedge j \wedge k ; \sigma, \mu \in V \\
x^{2}=[x, y]=(x, y, z)=1, \quad x \in \bar{V} . W . U, \quad y, z \in \mathcal{F} .
\end{gathered}
$$

Pela definição $\mathcal{Z}(\mathcal{F})=\bar{V} . W . U$. Agora sejam $v, w \in \mathcal{F}$ da forma $v=v_{0} z_{0}$ e $w=w_{0} z_{1}$, onde $v_{0}, w_{0} \in V$ e $z_{0}, z_{1} \in \mathcal{Z}(\mathcal{F})$. Assim, o produto de $v$ por $w$ é dado por: $v \cdot w=\left(v_{0} \cdot w_{0}\right) \cdot z_{0} \cdot z_{1}$.

Nas demonstrações a seguir usamos a notação mais concisa, para todos $\sigma, \mu, \lambda \in V$ :

$$
\begin{aligned}
{[\sigma, \mu, \lambda] } & =\prod_{i \in \sigma, j \in \mu, k \in \lambda j<k} i \wedge j \wedge k \\
{[\sigma, \mu] } & =\prod_{i \in \sigma, j \in \mu, i>j} i \wedge j \\
\{\sigma, \mu\} & =\left\{\begin{array}{cll}
\prod_{i \in \sigma \cap \mu} \bar{i}, & \text { se } & \sigma \cap \mu \neq \emptyset \\
1, & \text { se } & \sigma \cap \mu=\emptyset
\end{array}\right.
\end{aligned}
$$

Lema 1.3.1. Com as definições acima, em $\mathcal{F}$ valem as seguintes igualdades, para todos $\sigma, \mu, \lambda \in V:$

$$
\begin{gathered}
\{\sigma, \mu\}\{\sigma, \lambda\}\{\sigma \Delta \mu, \lambda \Delta \sigma\}=\{\lambda, \mu\}\{\sigma, \lambda \Delta \mu\}\{\sigma \Delta \mu \Delta \lambda, \sigma\} \\
{[\sigma, \mu][\lambda, \sigma][\sigma \Delta \mu, \lambda \Delta \sigma]=[\mu, \lambda][\sigma, \lambda \Delta \mu][\sigma \Delta \mu \Delta \lambda, \sigma]} \\
{[\lambda \Delta \tau, \mu, \sigma]=[\lambda, \mu, \sigma][\tau, \mu, \sigma]} \\
{[\sigma, \lambda \Delta \tau, \mu]=[\sigma, \lambda, \mu][\sigma, \tau, \mu]} \\
{[\sigma, \mu, \lambda \Delta \tau]=[\sigma, \mu, \lambda][\sigma, \mu, \tau]}
\end{gathered}
$$




$$
\begin{gathered}
{[\sigma, \lambda, \sigma][\sigma, \sigma, \lambda]=1} \\
{[\sigma, \lambda, \mu][\mu, \lambda, \sigma][\sigma, \mu, \lambda][\mu, \sigma, \lambda]=1} \\
{[\sigma \Delta \mu, \lambda \Delta \sigma, \lambda \Delta \sigma][\sigma, \mu, \mu][\lambda, \sigma, \sigma][\sigma \Delta \mu \Delta \lambda, \sigma, \sigma][\sigma, \mu \Delta \lambda, \mu \Delta \lambda][\mu, \lambda, \lambda]=1}
\end{gathered}
$$

Demonstração. Denotemos

$$
\begin{aligned}
& \xi_{1}=\sigma \cap \lambda \cap \mu, \quad \xi_{2}=(\sigma \cap \mu) \backslash \lambda, \quad \xi_{3}=(\sigma \cap \lambda) \backslash \mu, \quad \xi_{4}=\sigma \backslash(\mu \cup \lambda) \\
& \xi_{5}=(\lambda \cap \mu) \backslash \sigma, \quad \xi_{6}=\mu \backslash(\sigma \cup \lambda), \quad \xi_{7}=\lambda \backslash(\sigma \cup \mu) .
\end{aligned}
$$

Escrevemos $i, i j=(i j), i j k=(i j k),\{i, j\},\{i j, k\},[i, j],[i j, k],[i, j, k]$ e $[i p, j, k]$ ao invés $\operatorname{de} \xi_{i}, \xi_{i} \cup \xi_{j}, \xi_{i} \cup \xi_{j} \cup \xi_{k},\left\{\xi_{i}, \xi_{j}\right\},\left\{\xi_{i} \cup \xi_{j}, \xi_{k}\right\},\left[\xi_{i}, \xi_{j}\right],\left[\xi_{i} \cup \xi_{j}, \xi_{k}\right],\left[\xi_{i}, \xi_{j}, \xi_{k}\right]$ e $\left[\xi_{i} \cup \xi_{p}, \xi_{j}, \xi_{k}\right]$, respectivamente.

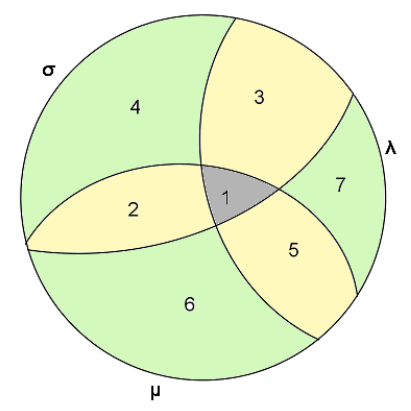

Figura 1.1: Diagrama representativo de $\sigma, \lambda$ e $\mu$

Para provarmos a igualdade (1.11) vejamos primeiramente que

$$
\{\sigma \cup \mu, \lambda\}=\{\sigma, \lambda\}\{\mu, \lambda\}, \quad \forall \sigma, \mu, \lambda \in V .
$$

De fato, basta observar que $\{\sigma \cup \mu, \lambda\}=\prod_{i \in(\sigma \cup \mu) \cap \lambda} \bar{i}=\prod_{i \in(\sigma \cap \lambda) \cup(\mu \cap \lambda)} \bar{i}=\prod_{i \in \sigma \cap \lambda} \bar{i} \cdot \prod_{i \in \mu \cap \lambda} \bar{i}$. 
Lembremos do fato

$$
\{\sigma, \mu\}=1 \quad \text { se } \sigma \cap \mu=\emptyset, \quad \forall \sigma, \mu \in V
$$

Assim, por (1.17) e (1.18), podemos reescrever a parte direita de (1.11) da seguinte maneira: $\{1,1\}\{5,5\}\{3,3\}\{2,2\}\{4,4\}\{1,1\}=\{5,5\}\{3,3\}\{2,2\}\{4,4\}$.

Com efeito:

(i)

$$
\begin{aligned}
\{\sigma \Delta \mu \Delta \lambda, \sigma\} & =\{[\sigma \backslash(\mu \Delta \lambda)] \cup[(\mu \Delta \lambda) \backslash \sigma], \sigma\} \\
& \stackrel{1.17}{=}\{\sigma \backslash(\mu \Delta \lambda), \sigma\}\{(\mu \Delta \lambda) \backslash \sigma, \sigma\} \\
= & \{[\sigma \backslash(\mu \cup \lambda)] \cup[\sigma \cap \lambda \cap \mu], \sigma\}\{[\mu \backslash(\lambda \cup \sigma)] \cup[\lambda \backslash(\mu \cup \sigma)], \sigma\} \\
& \stackrel{1.17}{=}\left\{\xi_{4}, \sigma\right\}\left\{\xi_{1}, \sigma\right\}\left\{\xi_{6}, \sigma\right\}\left\{\xi_{7}, \sigma\right\} .
\end{aligned}
$$

Observe que $\sigma \cap \xi_{4}=\xi_{4}$, então $\left\{\xi_{4}, \sigma\right\}=\left\{\xi_{4}, \xi_{4}\right\}=\{4,4\}$. Analogamente, $\left\{\xi_{1}, \sigma\right\}=\left\{\xi_{1}, \xi_{1}\right\}=\{1,1\}$. Observe também que $\xi_{6} \cap \sigma=\emptyset$ e $\xi_{7} \cap \sigma=\emptyset$, então por (1.18) temos $\left\{\xi_{6}, \sigma\right\}=\left\{\xi_{7}, \sigma\right\}=1$.

Portanto, $\{\sigma \Delta \mu \Delta \lambda, \sigma\}=\{4,4\}\{1,1\}$.

(ii)

$$
\begin{aligned}
\{\sigma, \lambda \Delta \mu\} \stackrel{1.17}{=}\{\sigma, \lambda \backslash \mu\}\{\sigma, \mu \backslash \lambda\} \\
=\{\sigma \cap(\lambda \backslash \mu), \sigma \cap(\lambda \backslash \mu)\}\{\sigma \cap(\mu \backslash \lambda), \sigma \cap(\mu \backslash \lambda)\} \\
=\{3,3\}\{2,2\} .
\end{aligned}
$$

Na última igualdade usamos a igualdade $x \cap(y \backslash z)=(x \cap y) \backslash z$, para quaisquer $x, y, z$ conjuntos. 
(iii) Temos $\lambda \cap \mu=(\lambda \cap \mu \cap \sigma) \cup((\lambda \cap \mu) \backslash \sigma)$, então

$$
\{\lambda, \mu\}=\{\lambda \cap \mu, \mu\}=\left\{\xi_{1} \cup \xi_{5}, \mu\right\}=\left\{\xi_{1}, \mu\right\}\left\{\xi_{5}, \mu\right\}=\{1,1\}\{5,5\} .
$$

Assim, por (i), (ii) e (iii), temos

$$
\{\lambda, \mu\}\{\sigma, \lambda \Delta \mu\}\{\sigma \Delta \mu \Delta \lambda, \sigma\}=\{1,1\}\{5,5\}\{3,3\}\{2,2\}\{4,4\}\{1,1\} .
$$

Por definição, $\{1,1\}\{1,1\}=1$, já que $\{1,1\} \in \bar{V}$. Portanto,

$$
\{\lambda, \mu\}\{\sigma, \lambda \Delta \mu\}\{\sigma \Delta \mu \Delta \lambda, \sigma\}=\{5,5\}\{3,3\}\{2,2\}\{4,4\}
$$

Agora vejamos que a parte esquerda de (1.11) pode ser reescrita da mesma maneira.

$\operatorname{Temos}\{\sigma \Delta \mu, \lambda \Delta \sigma\}=\{(\sigma \Delta \mu) \cap(\lambda \Delta \sigma), \lambda \Delta \sigma\}=\{[\sigma \backslash(\mu \cup \lambda)] \cup[(\lambda \cap \mu) \backslash \sigma], \lambda \Delta \sigma\}$. $\operatorname{Logo}\{\sigma \Delta \mu, \lambda \Delta \sigma\}=\{\sigma \backslash(\mu \cup \lambda), \lambda \Delta \sigma\}\{(\lambda \cap \mu) \backslash \sigma, \lambda \Delta \sigma\}=\{4,4\}\{5,5\}$. Além disso, $\{\sigma, \mu\}=\{\sigma \cap \mu, \mu\}=\{(\sigma \cap \mu \cap \lambda) \cup[(\sigma \cap \mu) \backslash \lambda], \mu\}=\{1,1\}\{2,2\}$ e, analogamente, provamos $\{\sigma, \lambda\}=\{1,1\}\{3,3\}$.

Portanto, $\{\sigma, \mu\}\{\sigma, \lambda\}\{\sigma \Delta \mu, \lambda \Delta \sigma\}=\{2,2\}\{3,3\}\{4,4\}\{5,5\}$.

Agora provaremos a igualdade (1.12). Temos $[\sigma \cup \mu, \lambda]=[\sigma, \lambda][\mu, \lambda]$, se $\sigma \cap \mu=\emptyset$. Pelo diagrama 1.1, podemos reescrever $\sigma, \lambda$ e $\mu$ da seguinte forma: $\sigma=(1234), \lambda=(1357)$ e $\mu=(1256)$. Logo, as partes direita e esquerda de (1.12) podem ser reescritas como um produto de $[i, j], i, j=1, \ldots, 7$. Como $[i, j]^{2}=1$, a igualdade (1.12) vale.

Para provarmos a relação (1.13), observemos que

$$
[\lambda \cup \tau, \mu, \sigma]=[\lambda, \mu, \sigma][\tau, \mu, \sigma], \text { se } \lambda \cap \tau=\emptyset \text {. }
$$


Portanto,

$$
\begin{aligned}
{[\lambda \Delta \tau, \mu, \sigma] } & =[(\lambda \backslash \tau) \cup(\tau \backslash \lambda), \mu, \sigma]=[\lambda \backslash \tau, \mu, \sigma][\tau \backslash \lambda, \mu, \sigma] \\
& =[\lambda \backslash \tau, \mu, \sigma][\lambda \cap \tau, \mu, \sigma][\lambda \cap \tau, \mu, \sigma][\tau \backslash \lambda, \mu, \sigma] \\
& =[\lambda, \mu, \sigma][\tau, \mu, \sigma] .
\end{aligned}
$$

Analogamente, prova-se as outras duas relações:

$$
[\sigma, \lambda \Delta \tau, \mu]=[\sigma, \lambda, \mu][\sigma, \tau, \mu] \quad \text { e } \quad[\sigma, \mu, \lambda \Delta \tau]=[\sigma, \mu, \lambda][\sigma, \mu, \tau]
$$

Vamos provar 1.14 e 1.15 no caso $\sigma \cap \mu=\sigma \cap \lambda=\mu \cap \lambda=\emptyset$.

Primeiramente consideremos $i, k \in \sigma$ e $j \in \lambda$. Suponha $i<k$. Temos que analisar três casos: 1) $i<j<k, 2) i<k<j$ e 3) $j<i<k$. No caso 1), o produto $i \wedge j \wedge k$ aparece duas vezes em $[\sigma, \lambda, \sigma][\sigma, \sigma, \lambda]$, como um fator de $[\sigma, \lambda, \sigma](i \wedge j \wedge k)$ e como um fator de $[\sigma, \sigma, \lambda](k \wedge i \wedge j)$. No caso 2) temos um fator da forma $i \wedge k \wedge j . k \wedge i \wedge j$ em $[\sigma, \sigma, \lambda]$ e não há fatores não-triviais em $[\sigma, \lambda, \sigma] \operatorname{com} i, j, k$, enquanto que no caso 3 ) temos o fator $k \wedge j \wedge i . i \wedge j \wedge k$ em $[\sigma, \lambda, \sigma]$ e não há fatores não-triviais em $[\sigma, \sigma, \lambda]$, com $i, j, k$. Os casos em que $k<i$ são análogos. Portanto, vale a relação (1.14) no caso particular $\sigma \cap \lambda=\emptyset$.

Agora sejam $i, j, k$ elementos de $\sigma, \mu$ e $\lambda$ respectivamente. Consideramos os mesmos três casos para provar a relação (1.15) no caso particular. Em qualquer caso teremos o fator $i \wedge j \wedge k$ exatamente duas vezes em $[\sigma, \lambda, \mu][\mu, \lambda, \sigma][\sigma, \mu, \lambda][\mu, \sigma, \lambda]$. Por exemplo, no caso 1) não há fatores com $i, j, k$ em $[\sigma, \lambda, \mu]$ e $[\mu, \lambda, \sigma]$ e há um fator $i \wedge j \wedge k$ em $[\sigma, \mu, \lambda]$ e um fator $j \wedge i \wedge k$ em $[\mu, \sigma, \lambda]$.

Para provarmos a relação (1.14) no caso geral consideramos novamente $\sigma=(1234)$ e $\lambda=(1357)$. Por $(1.19)$ o termo $[\sigma, \lambda, \sigma]=[1324,1357,1324]$ por ser reescrito como

$$
[13,13,13][13,13,24][13,57,13][13,57,24][24,13,13][24,13,24][24,57,13][24,57,24] \text {. }
$$


Da mesma forma o termo $[\sigma, \sigma, \lambda]=[1324,1324,1357]$ por ser reescrito como

$[13,13,13][13,13,57][13,24,13][13,24,57][24,13,13][24,13,57][24,24,13][24,24,57]$.

Por definição, $13 \cap 57=13 \cap 24=24 \cap 57=\emptyset$. Logo, como as relações (1.14) e (1.15) valem neste caso particular, obtemos $[\sigma, \lambda, \sigma][\sigma, \sigma, \lambda]=1$ no caso geral.

Vamos obter a relação (1.15) no caso geral. Por (1.14) temos

$$
[\sigma \Delta \mu, \lambda, \sigma \Delta \mu][\sigma \Delta \mu, \sigma \Delta \mu, \lambda]=1
$$

Aplicando (1.13) obtemos a relação desejada.

Por último, a relação (1.16) é obtida diretamente das relações (1.13), (1.14) e (1.15).

Teorema 1.3.1. O conjunto $\mathcal{F}$ definido acima é loop de Moufang na variedade $\mathcal{E}$.

Demonstração. Pela própria definição de $\mathcal{F}$ temos $\mathcal{F} \in \mathcal{E}$. Sejam $\sigma, \mu, \lambda \in V$. Vamos provar a seguinte identidade de Moufang:

$$
(\sigma \cdot \mu) \cdot(\lambda \cdot \sigma)=(\sigma \cdot(\mu \cdot \lambda)) \cdot \sigma
$$

Por 1.9:

$$
(\sigma \cdot \mu) \cdot(\lambda . \sigma)=((\sigma \Delta \mu) \cdot\{\sigma, \mu\} \cdot[\sigma, \mu] \cdot[\sigma, \mu, \mu]) \cdot((\lambda \Delta \sigma) \cdot\{\lambda, \sigma\} \cdot[\lambda, \sigma] \cdot[\lambda, \sigma, \sigma])
$$

Por 1.9 e 1.10:

$$
\begin{aligned}
(\sigma \cdot \mu) \cdot(\lambda . \sigma)= & (\mu \Delta \lambda) \cdot\{\sigma \Delta \mu, \lambda \Delta \sigma\} \cdot\{\sigma, \mu\} \cdot\{\lambda, \sigma\} . \\
& {[\sigma \Delta \mu, \lambda \Delta \sigma] \cdot[\sigma, \mu] \cdot[\lambda, \sigma] \cdot[\sigma, \mu, \mu] . } \\
& {[\sigma \Delta \mu, \lambda \Delta \sigma, \lambda \Delta \sigma] \cdot[\lambda, \sigma, \sigma] }
\end{aligned}
$$


Por outro lado,

$$
\begin{aligned}
& (\sigma \cdot(\mu . \lambda)) . \sigma \quad \stackrel{1.9}{=} \quad(\sigma \cdot(\mu \Delta \lambda) \cdot\{\mu, \lambda\} \cdot[\mu, \lambda] \cdot[\mu, \lambda, \lambda]) \cdot \sigma \\
& \stackrel{1.9}{=}(\sigma \Delta(\mu \Delta \lambda) \cdot\{\sigma, \mu \Delta \lambda\} \cdot\{\mu, \lambda\} \cdot[\mu, \lambda] . \\
& [\sigma, \mu \Delta \lambda] \cdot[\sigma, \mu \Delta \lambda, \mu \Delta \lambda] \cdot[\mu, \lambda, \lambda]) \cdot \sigma \\
& \stackrel{1.9-1.10}{=}(\mu \Delta \lambda) \cdot\{\sigma \Delta \mu \Delta \lambda, \sigma\} \cdot\{\sigma, \mu \Delta \lambda\} \cdot\{\mu, \lambda\} . \\
& {[\sigma \Delta \mu \Delta \lambda, \sigma] \cdot[\sigma, \mu \Delta \lambda] \cdot[\mu, \lambda] \cdot[\mu, \lambda, \lambda] .} \\
& {[\sigma \Delta \mu \Delta \lambda, \sigma, \sigma] \cdot[\sigma, \mu \Delta \lambda, \mu \Delta \lambda]}
\end{aligned}
$$

A igualdade 1.20 agora segue do Lema 1.3.1.

Consideremos um subloop de $\mathcal{F}$, denotado por $\mathcal{F}_{n}$ e com geradores $\left\{i \in I_{n}\right\}$, onde $I_{n}=\{1, \ldots, n\}$.

Definição 1.3.1. Um loop $F_{n}=\left\langle x_{1}, \ldots, x_{n}\right\rangle$ é um loop livre em $\mathcal{E}$ se, e somente se, para todo loop de Moufang $B \in \mathcal{E}$ com $B=\left\langle b_{1}, \ldots, b_{n}\right\rangle$, existe um único morfismo sobrejetor $\varphi: F_{n} \longrightarrow B$ tal que $\varphi\left(x_{i}\right)=b_{i}$.

Lema 1.3.2. Para todo $F_{n} \in \mathcal{E}$, com conjunto gerador $\left\{x_{1}, \ldots, x_{n}\right\}$, e para todo $z \in \mathcal{Z}\left(F_{n}\right)$, existem $\xi_{1}, \ldots, \xi_{n}, \xi_{i j}, \xi_{i j k}$, com $i, j, k=1, \ldots, n$ tais que

$$
z=\prod_{i=1}^{n}\left(x_{i}^{2}\right)^{\xi_{i}} \cdot \prod_{i<j}\left[x_{i}, x_{j}\right]^{\xi_{i j}} \cdot \prod_{i<j<k}\left(x_{i}, x_{j}, x_{k}\right)^{\xi_{i j k}}
$$

Demonstração. Sejam $\left.\left.x=\left(x_{i_{1}} x_{i_{2}}\right) x_{i_{3}}\right) \ldots\right) x_{i_{s}} \in F_{n}$, com $i_{1}<i_{2}<\cdots<i_{s}$ e $s \leq n$, e $z \in \mathcal{Z}\left(F_{n}\right)$. Usaremos a notação $x=x_{i_{1}} x_{i_{2}} \ldots x_{i_{s}}$. Definimos o comprimento de $x$, denotado por $|x|$, como sendo $s$.

Para provarmos que qualquer $z \in \mathcal{Z}\left(F_{n}\right)$ é da forma 1.21 faremos indução sobre $s$.

Suponhamos $s=2$, ou seja, considere $z=x^{2}$ ou $z=[x, y]$ ou $z=(x, y, w)$, para todos $y, w \in F_{n}$. Neste caso, o resultado sai diretamente das proposições 1.1.2 e 1.1.3, 
pois temos, em particular,

$$
\begin{aligned}
\left(x_{i} x_{j}\right)^{2} & =x_{i}^{2} x_{j}^{2}\left[x_{i}, x_{j}\right] ; \\
{\left[x_{i} x_{j}, x_{k}\right] } & =\left[x_{i}, x_{k}\right]\left[x_{j}, x_{k}\right]\left(x_{i}, x_{j}, x_{k}\right) ; \\
\left(x_{i} x_{j}, x_{k}, x_{p}\right) & =\left(x_{i}, x_{k}, x_{p}\right)\left(x_{j}, x_{k}, x_{p}\right) .
\end{aligned}
$$

Suponhamos o resultado válido para $s-1$ e provemos que vale para $s$. Sejam $y, w \in F_{n}$. Analisaremos 3 casos:

Caso $z=(x, y, w)$ : Neste caso, $z=\left(\left(x_{i_{1}} \ldots x_{i_{s-1}}\right) x_{i_{s}}, y, w\right)$. Pela proposição 1.1.2 temos que $z=\left(x_{i_{1}} \ldots x_{i_{s-1}}, y, w\right)\left(x_{i_{s}}, y, w\right)$ e por hipótese de indução, obtemos $z=\left(x_{i_{1}}, y, w\right) \ldots\left(x_{i_{s}}, y, w\right)$. Analogamente, prova-se para $z=(y, x, w)$ e $z=(y, w, x)$.

Caso $z=[x, y]$ : Aqui temos $z=[x, y]=\left[\left(x_{i_{1}} \ldots x_{i_{s-1}}\right) x_{i_{s}}, y\right]$. Novamente pela Proposição 1.1.2, obtemos $z=\left[x_{i_{1}} \ldots x_{i_{s-1}}, y\right]\left[x_{i_{s}}, y\right]\left(x_{i_{1}} \ldots x_{i_{s-1}}, x_{i_{s}}, y\right)$. Pela hipótese de indução, $z=\left[x_{i_{1}}, y\right] \ldots\left[x_{i_{s}}, y\right]\left(x_{i_{1}}, x_{i_{2}}, y\right) \ldots\left(x_{i_{1}}, x_{i_{s}}, y\right) \ldots\left(x_{i_{s-1}}, x_{i_{s}}, y\right)$. É análoga a demonstração para $z=[y, x]$.

Caso $z=x^{2}$ : Temos neste caso $z=\left(x_{i_{1}} \ldots x_{i_{s-1}}\right)^{2}\left(x_{i_{s}}\right)^{2}\left[x_{i_{1}} \ldots x_{i_{s-1}}, x_{i_{s}}\right]$, pela Proposição 1.1.3. Também aplicamos a hipótese de indução e assim obtemos:

$$
z=x_{i_{1}}^{2} \ldots x_{i_{s-1}}^{2} x_{i_{s}}^{2}\left[x_{i_{1}}, x_{i_{2}}\right] \ldots\left[x_{i_{1}}, x_{i_{s}}\right] \ldots\left[x_{i_{s-1}}, x_{i_{s}}\right]\left(x_{i_{1}}, x_{i_{2}}, x_{i_{3}}\right) \ldots\left(x_{i_{s-2}}, x_{i_{s-1}}, x_{i_{s}}\right) .
$$

Lema 1.3.3. Seja $F_{n} \in \mathcal{E}, F_{n}=\left\langle x_{1}, \ldots, x_{n}\right\rangle$ um loop com $n$ geradores e o morfismo sobrejetor $\varphi: F_{n} \longrightarrow \mathcal{F}_{n}$ tal que $\varphi\left(x_{i}\right)=i$. Então $\operatorname{ker} \varphi \subseteq \mathcal{Z}\left(F_{n}\right)$.

Demonstração. Considere $\psi$ um isomorfismo entre os $\mathbf{F}_{2}$-espaços $F_{n} / \mathcal{Z}\left(F_{n}\right)$ e $\mathcal{F}_{n} / \mathcal{Z}\left(\mathcal{F}_{n}\right)$. Seja $y \in \operatorname{Ker} \varphi$, então $\psi(\bar{y})=\overline{1}_{\mathcal{F}_{n}}$. Como $\psi\left(\overline{1}_{F_{n}}\right)=\overline{1}_{\mathcal{F}_{n}}$, então $\psi(\bar{y})=\psi\left(\overline{1}_{F_{n}}\right)$, mas $\psi$ é bijeção, portanto $\bar{y}=\overline{1}_{F_{n}}$, ou seja, $y \in \mathcal{Z}\left(F_{n}\right)$.

Teorema 1.3.2. $\mathcal{F}_{n}$ é um $\mathcal{E}$-loop livre de posto $n$. 
Demonstração. Seja $F_{n} \in \mathcal{E}, F_{n}=\left\langle x_{1}, \ldots, x_{n}\right\rangle$ um loop livre com $n$ geradores, então pela definição de loop livre existe um único morfismo sobrejetor $\varphi: F_{n} \longrightarrow \mathcal{F}_{n}$ tal que $\varphi\left(x_{i}\right)=i$.

Queremos mostrar que $\mathcal{F}_{n}$ é livre, para isso, basta mostrar que $\varphi$ é injetora. Vejamos que $\operatorname{Ker} \varphi=1$.

Seja $x \in \operatorname{Ker} \varphi$. Pelos Lemas 1.3 .2 e 1.3.3, existem $\xi_{1}, \ldots, \xi_{n}, \xi_{i j}, \xi_{i j k}$, $i, j, k=1, \ldots, n$ tais que

$$
x=\prod_{i=1}^{n}\left(x_{i}^{2}\right)^{\xi_{i}} \cdot \prod_{i<j}\left[x_{i}, x_{j}\right]^{\xi_{i j}} \cdot \prod_{i<j<k}\left(x_{i}, x_{j}, x_{k}\right)^{\xi_{i j k}}
$$

Logo:

$$
\varphi(x)=\prod_{i=1}^{n}\left(i^{2}\right)^{\xi_{i}} \cdot \prod_{i<j}[i, j]^{\xi_{i j}} \cdot \prod_{i<j<k}(i, j, k)^{\xi_{i j k}}=1,
$$

o que nos dá,

$$
\prod_{i=1}^{n}\left(i^{2}\right)^{\xi_{i}}=\prod_{i<j}[i, j]^{\xi_{i j}}=\prod_{i<j<k}(i, j, k)^{\xi_{i j k}}=1,
$$

e assim, $\xi_{i}=\xi_{i j}=\xi_{i j k}=0$. Portanto, $x=1$, como queríamos.

Portanto, $\mathcal{F}_{n} \cong F_{n}$ e consequentemente, $\mathcal{F}_{n}$ é loop livre.

Corolário 1.3.1. Para qualquer loop de código L de posto $n$ existe um homomorfismo $\varphi: \mathcal{F}_{n} \longrightarrow L$ tal que $\varphi\left(\mathcal{F}_{n}\right)=L$ e $\operatorname{codim}_{Z\left(\mathcal{F}_{n}\right)} \operatorname{ker}(\varphi)=1$.

Demonstração. Sejam $x_{1}, \ldots, x_{n}$ geradores livres de $\mathcal{F}_{n}$ e $v_{1}, \ldots, v_{n}$ geradores de $L$. Definimos $\varphi: \mathcal{F}_{n} \rightarrow L$ tal que $\varphi\left(x_{i}\right)=v_{i}, i=1, \ldots, n$. Então $K=\operatorname{ker} \varphi \subset Z\left(\mathcal{F}_{n}\right)$ and $\varphi\left(Z\left(\mathcal{F}_{n}\right)\right)=\{ \pm 0\} \subset L$. Logo $\operatorname{codim}_{Z\left(\mathcal{F}_{n}\right)} \operatorname{ker}(\varphi)=1$.

Proposição 1.3.1. Para todo $\boldsymbol{F}_{2}$-subespaço $T \subset Z\left(\mathcal{F}_{n}\right)$ de codimensão 1 temos um loop de código $L(T)=\mathcal{F}_{n} / T$.

Demonstração. Pelo Teorema 1.3.2, temos $\mathcal{F}_{n} \in \mathcal{E}$, então para todo $x \in \mathcal{F}_{n}$ temos 
$x^{2} \in \mathcal{Z}\left(\mathcal{F}_{n}\right), \operatorname{logo} x^{2} \in T$ ou $x^{2} \notin T$. Logo para $x T \in \mathcal{F}_{n} / T$ temos $x^{2} T=T$ ou $x^{2} T \neq T$. Assim, se $x^{2}, y^{2} \notin T$ então $x^{2} T=y^{2} T$, ou seja, $\left|L^{2}\right| \leq 2$. Portanto, pelos Teoremas 1.2 .2 e 1.2.3, $L(T)=\mathcal{F}_{n} / T$ é loop de código.

Proposição 1.3.2. O loop $L(T)$ é um grupo se, e somente se, $T \supseteq U_{n}=\left(\mathcal{F}_{n}, \mathcal{F}_{n}, \mathcal{F}_{n}\right)=$ $\mathcal{F}_{n} \cap U$, onde $U=(\mathcal{F}, \mathcal{F}, \mathcal{F})$.

Demonstração. $(\Rightarrow)$ Suponha que $L(T)=\mathcal{F}_{n} / T$ é grupo, então para todo $\bar{x}, \bar{y}, \bar{z} \in$ $L(T)$ temos $(\bar{x} \bar{y}) \bar{z}=\bar{x}(\bar{y} \bar{z})$, isto é, $\overline{(x y) z}=\overline{x(y z)}$. Logo $[x(y z)]^{-1}(x y) z \in T$, mas $[x(y z)]^{-1}(x y) z=(x, y, z) \in U_{n}$. Portanto, $U_{n} \subseteq T$.

$(\Leftarrow)$ Para mostrarmos que $L(T)$ é grupo, basta mostrar que $[x(y z)]^{-1}(x y) z \in T$. Isto vem do fato de que $[x(y z)]^{-1}(x y) z=(x, y, z) \in U_{n} \subseteq T$.

Proposição 1.3.3. Sejam $T_{1}$ e $T_{2} \boldsymbol{F}_{2}$-subespaços de $Z\left(\mathcal{F}_{n}\right)$ de codimensão 1 , então $L\left(T_{1}\right) \cong L\left(T_{2}\right)$ se, e somente se, existe um automorfismo $\varphi$ de $\mathcal{F}_{n}$ tal que $T_{1}^{\varphi}=T_{2}$.

Demonstração. Seja $\varphi: \mathcal{F}_{n} \longrightarrow \mathcal{F}_{n}$ um automorfismo tal que $T_{1}^{\varphi}=T_{2}$, onde $T_{1}$ e $T_{2}$ são $\mathbf{F}_{2}$-subespaços de $Z\left(\mathcal{F}_{n}\right)$ de codimensão 1 . Definimos $\bar{\varphi}: \mathcal{F}_{n} / T_{1} \longrightarrow \mathcal{F}_{n} / T_{2}$ por $\bar{\varphi}(\bar{x})=\overline{\varphi(x)}$, para todo $\bar{x} \in \mathcal{F}_{n} / T_{1}$. Claramente, $\varphi$ é um isomorfismo entre loops de código.

Agora construímos o seguinte diagrama comutativo:

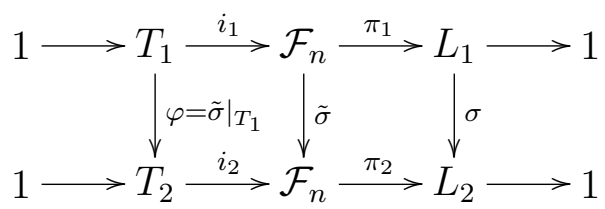

Suponhamos que $L_{j}=\mathcal{F}_{n} / T_{j}, \sigma: L_{1} \longrightarrow L_{2}$ é um isomorfismo, $\pi_{j}$ é um homomorfismo sobrejetor e $i_{j}$ é a aplicação inclusão, para $j=1,2$. Assim,

$$
1 \longrightarrow T_{j} \stackrel{i_{j}}{\longrightarrow} \mathcal{F}_{n} \stackrel{\pi_{j}}{\longrightarrow} L_{j} \longrightarrow 1
$$


é uma sequência exata curta, para $j=1,2$.

Sejam $x_{1}, \ldots, x_{n}$ o conjunto dos geradores livres de $\mathcal{F}_{n}$ e $v_{i}=\sigma\left(\pi_{1}\left(x_{i}\right)\right) \in L_{2}$.

Como $\pi_{2}$ é sobrejetor, existe $y_{i} \in \mathcal{F}_{n}$ tal que $\pi_{2}\left(y_{i}\right)=v_{i}$. Então existe um homomorfismo $\tilde{\sigma}: \mathcal{F}_{n} \rightarrow \mathcal{F}_{n}$ tal que $\tilde{\sigma}\left(x_{i}\right)=y_{i}$.

Como a parte direita do diagrama é comutativa pela construção, temos $\varphi\left(T_{1}\right)=\left.\tilde{\sigma}\right|_{T_{1}}\left(T_{1}\right) \subset T_{2}$.

Como $T_{1}$ e $T_{2}$ têm a mesma dimensão, basta mostrar que $\varphi$ é sobrejetora. De fato, seja $x \in T_{2}$, então $i_{2}(x)=x \in \mathcal{F}_{n}$, mas $\tilde{\sigma}$ é sobrejetora, logo existe $i \in \mathcal{F}_{n}$ tal que $\tilde{\sigma}(i)=x$. Por outro lado, $x \in \operatorname{Ker}\left(\pi_{2}\right)=\operatorname{Im}\left(i_{2}\right)$, pois as sequências das linhas do Diagrama 1.22 são exatas curtas. Daí $\pi_{2}(x)=T_{2}$, ou seja, $\pi_{2}(\tilde{\sigma}(i))=T_{2}$. Como a parte direita do Diagrama 1.22 é comutativa, temos $\sigma\left(\pi_{1}(i)\right)=T_{2}$. Então $\pi_{1}(i) \in \operatorname{Ker}(\sigma)=$ $\left\{T_{1}\right\}$, pois $\sigma$ é bijetora, ou seja, $\pi_{1}(i)=T_{1}$. Logo, $i \in \operatorname{Ker}\left(\pi_{1}\right)=\operatorname{Im}\left(i_{1}\right)=T_{1}$, como queríamos.

Denotemos por $\mathcal{L}_{n}$ o conjunto de todos os subespaços $T \subset Z\left(\mathcal{F}_{n}\right)$ de codimensão 1 tais que $T \nsupseteq U_{n}$. O grupo $G_{n}=A u t \mathcal{F}_{n}$ age sobre $\mathcal{L}_{n}$.

Seja $L_{n}$ o conjunto das $G_{n}$-órbitas correspondentes. Denotemos por $O_{T}$ a órbita de $T$, ou seja, $O_{T}=\left\{T^{\sigma} \mid \sigma \in G_{n}\right\}$.

Proposição 1.3.4. A correspondência $T \longrightarrow L(T)$ dá uma bijeção entre $L_{n}$ e o conjunto das classes de isomorfismo dos loops de código não associativos de posto $n$.

Demonstração. Sejam $T_{1}$ e $T_{2} \mathbf{F}_{2}$-subespaços de $\mathcal{Z}\left(\mathcal{F}_{n}\right)$ de codimensão 1 tais que $L\left(T_{i}\right)=\mathcal{F}_{n} / T_{i}$, para $i=1,2$. Denotamos por $\overline{L\left(T_{i}\right)}$ a classe dos loops de código não associativos de posto $n$ isomorfos a $L\left(T_{i}\right), i=1,2$. Suponhamos $\overline{L\left(T_{1}\right)}=\overline{L\left(T_{2}\right)}$. Então $L\left(T_{1}\right) \simeq L\left(T_{2}\right)$, logo existe $\varphi \in A u t \mathcal{F}_{n}$ tal que $T_{1}^{\varphi}=T_{2}$. Assim, $O_{T_{1}}=O_{T_{2}}$. Logo a função $O_{T} \longmapsto \overline{L(T)}$ é injetora. A sobrejetividade segue diretamente do Corolário 1.3.1. 
Seja $L$ um loop de código com conjunto gerador $X=\left\{x_{1}, \ldots, x_{n}\right\}$ e centro $\{1,-1\}$. Então definimos o vetor característico de $L$ por

$\lambda(L)=\lambda_{X}(L)=\left(\lambda_{1}, \ldots, \lambda_{n} ; \lambda_{12}, \ldots, \lambda_{1 n}, \ldots, \lambda_{(n-1) n} ; \lambda_{123}, \ldots, \lambda_{12 n}, \ldots, \lambda_{(n-2)(n-1) n}\right)$

onde $\lambda_{i}, \lambda_{i j}, \lambda_{i j k} \in \mathbf{F}_{2},(-1)^{\lambda_{i}}=x_{i}^{2}, \quad(-1)^{\lambda_{i j}}=\left[x_{i}, x_{j}\right]$ e $(-1)^{\lambda_{i j k}}=\left(x_{i}, x_{j}, x_{k}\right)$.

Com $\lambda(L) \neq 0$ podemos associar um subespaço $T=T_{\lambda}$ de $Z\left(\mathcal{F}_{n}\right)$ de codimensão 1 da seguinte maneira:

Escolhemos um elemento $\lambda_{i j k}=1$ e denotamos $x=x_{i} \wedge x_{j} \wedge x_{k}$. Então por definição $T_{\lambda}=\mathbf{F}_{2}\left\{x_{i}^{2}, x x_{j}^{2} \mid \lambda_{i}=0, \lambda_{j}=1\right\}+\mathbf{F}_{2}\left\{\left[x_{p}, x_{q}\right], x\left[x_{i}, x_{j}\right] \mid \lambda_{p q}=0, \lambda_{i j}=1\right\}+$ $\mathbf{F}_{2}\left\{\left(x_{i}, x_{j}, x_{k}\right), x\left(x_{p}, x_{q}, x_{l}\right) \mid \lambda_{i j k}=0, \lambda_{p q l}=1\right\}$.

Proposição 1.3.5. A aplicação $\lambda \longrightarrow T_{\lambda}$ é uma bijeção entre o conjunto de vetores característicos de loops de código não associativos de posto $n$ e os subespaços $T$ de $\mathcal{Z}=\mathcal{Z}\left(\mathcal{F}_{n}\right)$ de codimensão 1 tais que $U_{n} \not \subset T$.

Demonstração. Dados $\lambda$ e $\mu$, vetores característicos diferentes, pela definição de $T_{\lambda} \mathrm{e}$ $T_{\mu}$, temos $T_{\lambda} \neq T_{\mu}$. Agora, sabendo que $T \subset \mathcal{Z}$ é um subespaço de codimensão um (que não contém $(\mathrm{L}, \mathrm{L}, \mathrm{L})$ ), podemos recuperar um vetor característico $\lambda$.

Definimos $\lambda=\lambda(T)=\left(a_{1}, \ldots, a_{i, j} \ldots, a_{i, j, k} \ldots\right)$ tal que

$a_{i}=0 \Leftrightarrow x_{i}^{2} \in T$,

$a_{i, j}=0 \Leftrightarrow\left[x_{i}, x_{j}\right] \in T$,

$a_{i, j, k}=0 \Leftrightarrow\left(x_{i}, x_{j}, x_{k}\right) \in T$.

Claro que existe $p, q, r$ tais que $x=\left(x_{p}, x_{q}, x_{r}\right) \notin T$. Logo $Z=T \oplus \mathbf{F}_{2} x$ e, para qualquer $z \in Z$, temos $z \in T$ ou $z x \in T$. Isso significa

$$
\begin{aligned}
& a_{i}=1 \Leftrightarrow x_{i}^{2} x \in T, \\
& a_{i, j}=1 \Leftrightarrow\left[x_{i}, x_{j}\right] x \in T \mathrm{e} \\
& a_{i, j, k}=1 \Leftrightarrow\left(x_{i}, x_{j}, x_{k}\right) x \in T .
\end{aligned}
$$

Portanto, $T=T_{\lambda}$. 
Assim temos uma $G_{n}$-ação sobre o conjunto dos vetores característicos. A ação é definida por $\lambda^{\varphi}=\mu$ se, e somente se, $T_{\lambda}^{\varphi}=T_{\mu}$, para qualquer $\varphi \in G_{n}=\operatorname{Aut}\left(\mathcal{F}_{n}\right)$.

Sem perda de generalidade podemos supor $\lambda_{123}=1$. Neste caso, temos $2^{m}$ vetores característicos onde $m=n+\frac{n(n-1)}{2}+\frac{n(n-1)(n-2)}{6}-1$. Seja $\left\{O_{1}, \ldots, O_{k}\right\}$ um conjunto de $G_{n}$-órbitas de vetores característicos de loops de código de posto $n$. Então

$$
\sum_{i=1}^{k}\left|O_{i}\right|=2^{m} .
$$

Para um dado loop de código $L$, definimos $O u t L=$ Aut $L / N($ Aut $L)$, o grupo de automorfismos externos de $L$. Por definição, $N(A u t L)=\{\phi \in A u t L \mid \phi(x)= \pm x, \forall x \in L\}$.

Seja $G_{n}^{i}$ o grupo estabilizador de $\lambda \in O_{i}$, ou seja, $G_{n}^{i}=\left\{\varphi \in G_{n} \mid \lambda^{\varphi}=\lambda\right\}$. Notemos que $\varphi \in G_{n}^{i}$ induz um automorfismo do loop de código correspondente $L_{i}=\mathcal{F}_{n} / T_{\lambda}$.

Para a próxima proposição, definimos $\varphi \in \mathcal{Z}^{n} \subset G_{n}$, se, e somente se, $\varphi(i)=i z_{i}$, para todo $i \in\{1, \ldots, n\}$.

Proposição 1.3.6. Consideremos o conjunto

$$
\overline{G L_{n}(2)}=\left\{\varphi \in G_{n} \mid i^{\varphi} \subseteq V, \text { para todo } i \in\{1, \ldots, n\}\right\} \subset G_{n} \text { e } \mathcal{Z}=\mathcal{Z}\left(\mathcal{F}_{n}\right)
$$

\section{Então:}

i) $G_{n}=\overline{G L_{n}(2)} \cdot \mathcal{Z}^{n}$;

ii) $G_{n} / \mathcal{Z}^{n} \simeq G L_{n}(2)=\operatorname{Aut}_{\mathbf{F}_{2}} V e$

iii) $G_{n}^{i} / \mathcal{Z}^{n} \simeq \operatorname{Out}\left(L_{i}\right)$.

Demonstração. Seja $\varphi \in G_{n}$, então $\varphi(i)=\sigma_{i} . z_{i}$, para $\sigma_{i} \in V=\{1, \ldots, n\}$ e $z_{i} \in \mathcal{Z}$. Logo, para todo $\varphi \in G_{n}$, existe $\left\{\sigma_{1}, \ldots, \sigma_{n}\right\}$ uma base correspondente de $V$ e $\left(z_{1}, \ldots, z_{n}\right) \in \mathcal{Z}^{n}$. Temos que $\mathcal{F}_{n} / \mathcal{Z}\left(\mathcal{F}_{n}\right) \simeq V=\{1, \ldots, n\}$ é um $\mathbf{F}_{2}$-espaço ve- 
torial e, para $\varphi \in G_{n}$, temos um automorfismo $\tilde{\varphi}: \mathcal{F}_{n} / \mathcal{Z}\left(\mathcal{F}_{n}\right) \longrightarrow \mathcal{F}_{n} / \mathcal{Z}\left(\mathcal{F}_{n}\right)$ tal que $i^{\tilde{\varphi}}=\sigma_{i}$. Logo $\tilde{\varphi} \in G L(V)=G L_{n}(2)$.

Provemos agora que $G_{n}^{i} / \mathcal{Z}^{n} \simeq \operatorname{Out}\left(L_{i}\right)$. De fato, seja $\varphi \in G_{n}^{i}$, então $T_{\lambda}^{\varphi}=T_{\lambda}$, $\operatorname{logo} \tilde{\varphi}: \mathcal{F}_{n} / T_{\lambda} \longrightarrow \mathcal{F}_{n} / T_{\lambda}$ é um isomorfismo de loops de código. O homomorfismo $\pi_{i}: G_{n}^{i} \longrightarrow A u t\left(L_{i}\right)$ tal que $\pi_{i}(\varphi)=\tilde{\varphi}$, é sobrejetor (pela Proposição 1.3.3) com núcleo $K_{i}=\left\{\tau \in G_{n}^{i} \mid \tau\left(x_{j}\right)=x_{j} v_{j}, v_{j} \in T_{\lambda}\right\}$. Considere também o homomorfismo sobrejetor $\psi_{i}: \operatorname{Aut}\left(L_{i}\right) \longrightarrow \operatorname{Out}\left(L_{i}\right) . \operatorname{Logo} \pi=\psi_{i} \circ \pi_{i}: G_{n}^{i} \longrightarrow \operatorname{Out}\left(L_{i}\right)$ é um homomorfismo sobrejetor. Temos $\mathcal{Z}^{n} \subseteq \operatorname{ker}(\pi)$. Agora, seja $\varphi \in \operatorname{ker}(\pi)$. Se $\varphi \notin \mathcal{Z}^{n}$, existe $i^{\varphi} \neq i z_{i}$, para todo $z_{i} \in \mathcal{Z}$, então para $\pi(\varphi) \in \operatorname{Out}\left(L_{i}\right)$, temos $\pi(\varphi): i \longmapsto \pm i^{\varphi} \neq \pm i\left(i \bmod \left(T_{\lambda}\right)\right), \operatorname{logo} \pi \varphi \neq 1$ em $\operatorname{Out}\left(L_{i}\right) . A s s i m, k e r(\pi)=\mathcal{Z}^{n}$ e, portanto, $G_{n}^{i} / \mathcal{Z}^{n} \simeq \operatorname{Out}\left(L_{i}\right)$.

$\operatorname{Denotamos} G_{n}^{i} / \mathcal{Z}^{n}$ por $\operatorname{Stab}(\lambda)$.

Notemos que $\lambda(L)$ depende não somente de $L$ mas de uma escolha do conjunto gerador $X=\left\{x_{1}, \ldots, x_{n}\right\}$ e quando for necessário, denotaremos os vetores característicos por $\lambda_{X}(L)$.

Um problema importante na teoria de loops de código é encontrar critérios para decidir quando dois loops de código $L_{1}$ e $L_{2}$ com os dados vetores característicos $\lambda\left(L_{1}\right)$, $\lambda\left(L_{2}\right)$ são isomorfos.

Notemos que dois loops de código $L_{1}$ e $L_{2}$ são isomorfos se, e somente se, eles contêm os conjuntos geradores $X \subset L_{1}$ e $Y \subset L_{2}$ tais que $\lambda_{X}\left(L_{1}\right)=\lambda_{Y}\left(L_{2}\right)$.

Definição 1.3.2. Um 3-espaço é um $\mathbf{F}_{2}$-espaço $V$ com uma forma trilinear antisimétrica ( , , ) com valores em $\mathbf{F}_{2}$. Um 2-espaço é um 3-espaço $V$ com uma aplicação anti-simétrica $V \times V \longrightarrow \mathbf{F}_{2}$ tal que $[v, w u]=[w u, v]=[v, w][v, u](v, w, u)$.

Dado um loop de código $L$, podemos associar um 2-espaço $V=L / \mathcal{Z}(L)$ com uma 3-forma induzida pelo associador e a correspondente aplicação anti-simétrica induzida pelo comutador. 
Seja $V$ um 3-espaço. Por definição, o núcleo de $V$ é o subespaço

$$
N_{3}(V)=\{v \in V \mid(v, V, V)=1\} .
$$

Analogamente, para um 2-espaço $V$, definimos

$$
N_{2}(V)=\{v \in V \mid(v, V, V)=[v, V]=1\} .
$$

Definição 1.3.3. Para dois loops de código $L_{1}$ e $L_{2}$, definimos o produto de $L_{1}$ e $L_{2}$ como um loop de código $L_{1} * L_{2}=\frac{L_{1} \times L_{2}}{D}$, onde $D=\{1 \times 1,(-1) \times(-1)\}$ é uma diagonal do centro de $L_{1} \times L_{2}$.

Seja $L$ um outro loop de código, escrevemos $L_{1} \sim_{L} L_{2}$ se $L * L_{1} \simeq L * L_{2}$ e dizemos que $L_{1}$ e $L_{2}$ são L-equivalentes.

Lema 1.3.4. Dois loops de código $L_{1}$ e $L_{2}$ são isomorfos como 2-espaços se, e somente se, $L_{1} \sim_{\mathbb{Z}_{4}} L_{2}$.

Demonstração. Sejam $L_{1}$ e $L_{2} \mathbb{Z}_{4}$-equivalentes. Portanto, existe um isomorfismo $\sigma: \mathbb{Z}_{4} * L_{1} \longrightarrow \mathbb{Z}_{4} * L_{2}$. Definimos uma aplicação linear $\tau: L_{1} \longrightarrow L_{2}$ por $\tau(v)=w \in L_{2}$ se $\sigma(v)=a w$, onde $a \in \mathbb{Z}_{4}$. Como por definição $\left[a, L_{2}\right]=\left(a, L_{2}, L_{2}\right)=1$, então $\tau$ é um isomorfismo de $L_{1}$ e $L_{2}$ como 2-espaços.

Reciprocamente, se $\tau: L_{1} \longrightarrow L_{2}$ é um isomorfismo de loops de código como 2espaços, então a aplicação $\sigma: \mathbb{Z}_{4} * L_{1} \longrightarrow \mathbb{Z}_{4} * L_{2}$, onde $\sigma(a \times v)=b \times \tau(v)$ e $b=a$ se $v^{2}=\tau(v)^{2}, b=a c$ se $v^{2} \neq \tau(v)^{2}, a, b, c \in \mathbb{Z}_{4}, c^{2}=-1$, é um isomorfismo. 


\section{Capítulo 2}

\section{Classificação dos Loops de Código de posto 3 e 4}

\subsection{Loops de Código de posto 3}

Seja $L$ um loop de código não-associativo de posto 3 com geradores $a, b, c$. Logo $(a, b, c)=-1$. Com isso, podemos escrever o vetor característico correspondente $\lambda(L)$ da forma $\lambda(L)=\left(\lambda_{1}, \ldots, \lambda_{6}\right)$ onde

$$
\begin{gathered}
a^{2}=(-1)^{\lambda_{1}}, \quad b^{2}=(-1)^{\lambda_{2}}, \quad c^{2}=(-1)^{\lambda_{3}}, \\
{[a, b]=(-1)^{\lambda_{4}},[a, c]=(-1)^{\lambda_{5}}, \quad[b, c]=(-1)^{\lambda_{6}} .}
\end{gathered}
$$

Denotemos por $C_{1}^{3}, \ldots, C_{5}^{3}$ os loops de códigos com os seguintes vetores característicos 
correspondentes:

$$
\begin{aligned}
& \lambda\left(C_{1}^{3}\right)=(1,1,1,1,1,1) \\
& \lambda\left(C_{2}^{3}\right)=(0,0,0,0,0,0) \\
& \lambda\left(C_{3}^{3}\right)=(0,0,0,1,1,1) \\
& \lambda\left(C_{4}^{3}\right)=(1,1,0,0,0,0) \\
& \lambda\left(C_{5}^{3}\right)=(1,0,0,0,0,0)
\end{aligned}
$$

Teorema 2.1.1. Quaisquer dois loops da lista $\left\{C_{1}^{3}, \ldots, C_{5}^{3}\right\}$ são não isomorfos e qualquer loop de código não-associativo de posto 3 é isomorfo a um desta lista.

Demonstração. Sejam $a, b, c$ os geradores do loop de código $L$ não-associativo de posto 3. Logo $(a, b, c)=-1$. Existem duas possibilidades:

1. $[a, b]=1$;

2. $[a, b]=[a, c]=[b, c]=-1$.

No primeiro caso, podemos escolher geradores tais que $[a, b]=[a, c]=[b, c]=1$. De fato, se $[a, c]=-1$, então tomamos $x=b c$ e teremos $[a, x]=[a, b][a, c](a, b, c)=1$, pelo Teorema 1.1.4.

Se $[a, b]=[a, c]=1, \operatorname{mas}[b, c]=-1, \operatorname{temos}[a, a b]=[a, c]=[a b, c]=1$.

Fixados os geradores $a, b, c$ tais que $[a, b]=[a, c]=[b, c]=1$, teremos para $\lambda(L)$ quatro possibilidades: $(0,0,0,0,0,0),(1,1,0,0,0,0),(1,0,0,0,0,0),(1,1,1,0,0,0)$.

Observemos que os loops que correspondem aos dois últimos vetores característicos são isomorfos. De fato, seja $L_{1}$ o loop de código com conjunto gerador $X=\{a, b, c\}$ e com vetor característico correspondente $\lambda_{X}\left(L_{1}\right)=(1,1,1,0,0,0)$. Logo, para o conjunto gerador $Y=\{a, a b, a c\}$, temos $a^{2}=-1,(a b)^{2}=1,(a c)^{2}=1$, ou seja, $\lambda_{Y}\left(L_{1}\right)=(1,0,0,0,0,0)$.

No segundo caso, podem acontecer dois subcasos: 
i) $a^{2}=b^{2}=c^{2}=-1$ : Aqui temos $\lambda(L)=(1,1,1,1,1,1)$.

ii) $a^{2}=1$ : Supondo $b^{2}=-1$ ou $c^{2}=-1$ teremos $(a c)^{2}=(a b)^{2}=1$. Daí podemos escolher geradores $a, b, c$ tais que $a^{2}=b^{2}=c^{2}=1$, $\operatorname{logo} \lambda(L)=(0,0,0,1,1,1)$.

Proposição 2.1.1. Sejam $O_{1}^{3}, \ldots, O_{5}^{3}$ as órbitas dos loops de código $C_{1}^{3}, \ldots, C_{5}^{3}$ respectivamente. Então

$$
\begin{aligned}
& O_{1}^{3}=\{(111111)\} \\
& O_{2}^{3}=\{(000000),(000001),(000010),(000100),(001011),(010101),(100110)\} \\
& O_{3}^{3}=\{(000111),(001111),(010111),(011111),(100111),(101111),(110111)\} \\
& O_{4}^{3}=\{(001100),(010010),(011000),(011001),(011011),(011101),(100001), \\
&(101000),(101010),(101011),(101110),(110000),(110100),(110101), \\
&(110110),(111001),(111010),(111011),(111100),(111101),(111110)\} \\
& O_{5}^{3}=\{(000011),(000101),(000110),(001000),(001001),(001010),(001101),(001110),(010000),(010001),(010011),(010100),(010110),(011010), \\
&(011100),(011110),(100000),(100010),(100011),(100100),(100101), \\
&(101001),(101100),(101101),(110001),(110010),(110011),(111000)\}
\end{aligned}
$$

Demonstração. Seja $(a, b, c)$ um conjunto de geradores para cada $C_{i}^{3}, i=1, \ldots, 5$. Como vimos anteriormente, cada loop de código possui um vetor característico correspondente, dependendo do conjunto de geradores considerado. Com o conjunto de geradores fixado em cada caso, considere como representante de cada órbita $O_{i}^{3}$, $i=i, \ldots, 5$, os vetores $\lambda\left(C_{i}^{3}\right), i=1, \ldots, 5$, respectivamente. Para exibirmos todos os vetores que pertencem as órbitas de cada representante temos que exibir os conjuntos geradores diferentes formados a partir do conjunto gerador $(a, b, c)$ fixado. Vejamos na Tabela 2.1 todos os valores de quadrados e comutadores que cada vetor característico 
nos fornece, ou seja, os quadrados de cada elemento gerador e o comutador entre os elementos geradores. Cada coluna $i$ corresponde a um loop de código $C_{i}^{3}, i=1, \ldots, 5$.

\begin{tabular}{|r||r|r|r|r|r|}
\hline & 1 & 2 & 3 & 4 & 5 \\
\hline \hline$a^{2}$ & -1 & 1 & 1 & -1 & -1 \\
\hline$b^{2}$ & -1 & 1 & 1 & -1 & 1 \\
\hline$c^{2}$ & -1 & 1 & 1 & 1 & 1 \\
\hline$[a, b]$ & -1 & 1 & -1 & 1 & 1 \\
\hline$[a, c]$ & -1 & 1 & -1 & 1 & 1 \\
\hline$[b, c]$ & -1 & 1 & -1 & 1 & 1 \\
\hline
\end{tabular}

Tabela 2.1: Quadrados e Comutadores dos geradores $a, b, c$ fixados

Seja $\varphi \in G L_{3}(2)$ tal que $\varphi$ seja um automorfismo de $C_{i}^{3}$. Para cada $\varphi$ encontramos um novo conjunto de geradores de $C_{i}^{3}$, denotado por $(x, y, z)$, ou seja, $\left(a^{\varphi}=x, b^{\varphi}=\right.$ $\left.y, c^{\varphi}=z\right)$. Vejamos na Tabela 2.2 os quadrados de $x, y, z$ e os comutadores entre esses elementos, pois a partir desses valores podemos encontrar o vetor característico associado a $(x, y, z)$.

\begin{tabular}{|l|r|r|r|r|r|}
\hline & 1 & 2 & 3 & 4 & 5 \\
\hline$(a b)^{2}$ & -1 & 1 & -1 & 1 & -1 \\
$(a c)^{2}$ & -1 & 1 & -1 & -1 & -1 \\
$(b c)^{2}$ & -1 & 1 & -1 & -1 & 1 \\
$(a b c)^{2}$ & -1 & -1 & 1 & -1 & 1 \\
{$[a, a b]$} & -1 & 1 & -1 & 1 & 1 \\
{$[a, a c]$} & -1 & 1 & -1 & 1 & 1 \\
{$[a, b c]$} & -1 & -1 & -1 & -1 & -1 \\
{$[a, a b c]$} & -1 & -1 & -1 & -1 & -1 \\
{$[b, a b]$} & -1 & 1 & -1 & 1 & 1 \\
{$[b, a c]$} & -1 & -1 & -1 & -1 & -1 \\
{$[b, b c]$} & -1 & 1 & -1 & 1 & 1 \\
\hline
\end{tabular}

\begin{tabular}{|l|r|r|r|r|r|}
\hline & 1 & 2 & 3 & 4 & 5 \\
\hline$[b, a b c]$ & -1 & -1 & -1 & -1 & -1 \\
{$[c, a b]$} & -1 & -1 & -1 & -1 & -1 \\
{$[c, a c]$} & -1 & 1 & -1 & 1 & 1 \\
{$[c, b c]$} & -1 & -1 & 1 & 1 & 1 \\
{$[c, a b c]$} & -1 & 1 & -1 & -1 & -1 \\
{$[a b, a c]$} & -1 & 1 & -1 & 1 & 1 \\
{$[a b, b c]$} & -1 & 1 & -1 & 1 & 1 \\
{$[a b, a b c]$} & -1 & -1 & -1 & -1 & -1 \\
{$[a c, b c]$} & -1 & 1 & 1 & 1 & 1 \\
{$[a c, a b c]$} & -1 & -1 & -1 & -1 & -1 \\
{$[b c, a b c]$} & -1 & -1 & -1 & -1 & -1 \\
\hline
\end{tabular}

Tabela 2.2: Quadrados e Comutadores de $x, y$ e $z$

Na primeira órbita temos apenas o vetor $\lambda\left(C_{1}^{3}\right)=(111111)$, pois para quaisquer $x, y, z$ como acima, sempre teremos $x^{2}=y^{2}=z^{2}=-1$ e $[x, y]=[x, z]=[y, z]=-1$, como vemos pela tabela dada.

Para encontrarmos os vetores da órbita $O_{2}^{3}$, basta observar a Tabela 2.2 e concluir 
que os conjuntos geradores $(x, y, z)=(a, b, c),(a, b, a c),(a, b, b c),(a, b c, b),(a b c, a c, b c)$, $(a c, a b c, b c)$ e $(a c, b c, a b c)$ correspondem respectivamente aos vetores característicos (000000), (000001), (000010), (000100), (100110), (010101) e (001011). Estes vetores constituem a órbita $O_{2}^{3}$. Pela mesma tabela, obtemos os vetores de $O_{3}^{3}$. As possibilidades para $(x, y, z)$, neste caso, são $(a, b, c),(a b, a, c),(a, a b, c),(a, c, a b),(a b, a c, a)$, $(a b, a, a c)$ e $(a, a b, a c)$, com vetores característicos respectivamente dados por (000111), (100111), (010111), (001111), (110111), (101111) e (011111).

Analogamente, com simples cálculos, obtemos todos os vetores das órbitas $O_{4}^{3}$ e $O_{5}^{3}$. Veja no anexo, a Tabela A.1 mostra todas as possibilidades para $(x, y, z)$ com seus respectivos vetores característicos.

Notemos que $\left|O_{1}^{3}\right|=1,\left|O_{2}^{3}\right|=\left|O_{3}^{3}\right|=7,\left|O_{4}^{3}\right|=21,\left|O_{5}^{3}\right|=28$ e $\sum_{i=1}^{5}\left|O_{i}^{3}\right|=64=2^{6}$. Portanto,

$$
\begin{gathered}
\mid \text { Out } C_{1}^{3}|=| G L_{3}(2) \mid=168, \\
\left|\operatorname{Out}_{2}^{3}\right|=\left|\operatorname{Out}_{3}^{3}\right|=24, \\
\left|\operatorname{Out}_{4}^{3}\right|=8 \text { e }\left|\operatorname{Out}_{3}^{3}\right|=6 .
\end{gathered}
$$

Nas demonstrações a seguir usamos a notação $G R\left\{x_{1}, \ldots, x_{n}\right\}$ para denotar o grupo gerado pelos elementos $x_{1}, \ldots, x_{n}$.

Proposição 2.1.2. Na notação acima, temos:

1. $\mathrm{OutC}_{1}^{3} \simeq G L_{3}(2)$,

2. $\mathrm{Out} C_{2}^{3} \simeq S_{4}$,

3. $\mathrm{Out} C_{3}^{3} \simeq S_{4}$,

4. $\mathrm{OutC}_{4}^{3} \simeq D_{8}$, 
5. $\mathrm{Out} C_{5}^{3} \simeq S_{3}$.

Demonstração. 1. Sejam $a, b, c$ geradores de $L=C_{1}^{3}$ com vetor característico correspondente $\lambda=\lambda(L)=(111111)$, ou seja $a^{2}=b^{2}=c^{2}=-1$ e $[a, b]=$ $[a, c]=[b, c]=-1$. Pelas Tabelas 2.1 e 2.2 temos, para qualquer $\varphi \in G L_{3}(2)$, $\left(a^{\varphi}\right)^{2}=\left(b^{\varphi}\right)^{2}=\left(c^{\varphi}\right)^{2}=-1$ e $\left[a^{\varphi}, b^{\varphi}\right]=\left[a^{\varphi}, c^{\varphi}\right]=\left[b^{\varphi}, c^{\varphi}\right]=-1$.

2. Queremos encontrar todos os automorfismos externos do loop de código $L=C_{2}^{3}$. Com este objetivo, consideremos $\lambda=\lambda(L)=(000000)$ um vetor característico de $L$ representante da órbita $O_{2}^{3}$ e $(a, b, c)$ um conjunto de geradores para $L$ tais que $a^{2}=b^{2}=c^{2}=1$ e $[a, b]=[a, c]=[b, c]=1$.

Consideremos $\varphi_{1}, \ldots, \varphi_{6} \in G L_{3}(2)$ tais que

$$
\begin{gathered}
\varphi_{1}=i d \quad \varphi_{2}=\left(\begin{array}{lll}
1 & 0 & 0 \\
0 & 0 & 1 \\
0 & 1 & 0
\end{array}\right) \quad \varphi_{3}=\left(\begin{array}{lll}
0 & 1 & 0 \\
1 & 0 & 0 \\
0 & 0 & 1
\end{array}\right) \\
\varphi_{4}=\left(\begin{array}{lll}
0 & 0 & 1 \\
1 & 0 & 0 \\
0 & 1 & 0
\end{array}\right) \quad \varphi_{5}=\left(\begin{array}{lll}
0 & 1 & 0 \\
0 & 0 & 1 \\
1 & 0 & 0
\end{array}\right) \quad \varphi_{6}=\left(\begin{array}{lll}
0 & 0 & 1 \\
0 & 1 & 0 \\
1 & 0 & 0
\end{array}\right)
\end{gathered}
$$

Temos $\lambda^{\varphi_{i}}=\lambda$, para cada $i=1, \ldots, 6 . \operatorname{Logo} \varphi_{i} \in \operatorname{Stab}(\lambda)$, para cada $i=1, \ldots, 6$. Observemos que $G R\left\{\varphi_{1}, \ldots, \varphi_{6}\right\}=S_{3}$. Portanto, $S_{3} \subseteq \operatorname{Stab}(\lambda)$. Pela Proposição 2.1.1, em $O_{2}^{3}$ existem exatamente 7 códigos, então temos que mostrar que existem 24 automorfismos que fixam $\lambda$. Seja $\sigma \in G L_{3}(2)$ da forma $\sigma=\left(\begin{array}{lll}1 & 1 & 0 \\ 0 & 1 & 1 \\ 0 & 1 & 0\end{array}\right)$. Temos $\lambda^{\sigma}=\lambda$, ou seja, $(a b)^{2}=(b c)^{2}=b^{2}=[a b, b c]=[a b, b]=[b c, b]=1$. Logo, $\sigma \in \operatorname{Stab}(\lambda)$. 
Observemos que $\sigma^{3}=1$ e $\sigma^{2}=\left(\begin{array}{ccc}1 & 0 & 1 \\ 0 & 0 & 1 \\ 0 & 1 & 1\end{array}\right)$. Além disso, $\lambda^{\sigma^{2}}=\lambda$, ou seja, $(a c)^{2}=c^{2}=(b c)^{2}=[a c, c]=[a c, b c]=[c, b c]=1 . \log 0 \sigma^{2} \in \operatorname{Stab}(\lambda)$.

Logo, com cálculos diretos, se mostra que os elementos pertencentes aos conjuntos $\sigma S_{3}, S_{3} \sigma, \sigma^{2} S_{3}, S_{3} \sigma^{2}$, são automorfismos que fixam $\lambda$, ou seja, para todo $i=1, \ldots, 6$ temos $\left(\sigma \varphi_{i}\right),\left(\sigma^{2} \varphi_{i}\right),\left(\varphi_{i} \sigma\right),\left(\varphi_{i} \sigma^{2}\right) \in \operatorname{Stab}(\lambda)$. Destes conjuntos obtemos mais 16 estabilizadores diferentes. Para completar nosso grupo de estabilizadores considere $\left(\sigma \varphi_{3}\right) \sigma^{2}$ e $\left(\sigma^{2} \varphi_{5}\right) \sigma^{2}$.

Sabemos que Out $C_{2}^{3} \simeq \operatorname{Stab}(\lambda)$. Para provar que Out $C_{2}^{3} \simeq S_{4}$, basta mostrar que $\operatorname{Stab}(\lambda)=S_{4}$. De fato, primeiro denotemos $\varphi_{2}, \varphi_{3},\left(\sigma \varphi_{3}\right) \sigma^{2}$, respectivamente, por $\sigma_{1}, \sigma_{2}, \sigma_{3}$ e depois, com cálculos diretos mostramos que $\varphi_{2}^{2}=\varphi_{3}^{2}=$ $\left(\sigma \varphi_{3}\right) \sigma^{2}=1$ e valem as seguintes relações $\sigma_{1} \sigma_{2} \sigma_{1}=\sigma_{2} \sigma_{1} \sigma_{2}, \sigma_{2} \sigma_{3} \sigma_{2}=\sigma_{3} \sigma_{2} \sigma_{3} \mathrm{e}$ $\sigma_{1} \sigma_{3}=\sigma_{3} \sigma_{1}$. Logo, $\operatorname{Stab}(\lambda)=G R\left\{\sigma_{1}, \sigma_{2}, \sigma_{3} \mid \sigma_{i}^{2}=1, \sigma_{i} \sigma_{i+1} \sigma_{i}=\sigma_{i+1} \sigma_{i} \sigma_{i+1}, \sigma_{i} \sigma_{j}=\right.$ $\left.\sigma_{j} \sigma_{i}, j \neq i \pm 1, i=1,2,3\right\}=S_{4}$.

3. Sejam $a, b, c$ geradores do loop de código $L=C_{3}^{3}$ com vetor característico $\lambda=\lambda(L)=(000111)$. Temos que $\operatorname{Stab}(\lambda)$ é o grupo de todas as permutações do conjunto $X=\{a, b, c, a b c\}$. Para vermos isto considere $\varphi \in G L_{3}(2)$ tal que $\varphi(a)=x, \varphi(b)=y$ e $\varphi(c)=z$.

Temos que encontrar $(x, y, z)$ tais que $x^{2}=y^{2}=z^{2}=1$ e $[x, y]=[x, z]=$ $[y, z]=-1$. As Tabelas 2.1 e 2.2, nos dão todos os valores possíveis de $x, y, z$ que satisfazem as relações desejadas. Assim, $x, y, z \in\{a, b, c, a b c\}$. Consideremos $\sigma_{i} \in G L_{3}(2)$, para $i=1,2,3$, tais que $\sigma_{1}(a)=a, \sigma_{1}(b)=b, \sigma_{1}(c)=a b c$; $\sigma_{2}(a)=a, \sigma_{2}(b)=a b c, \sigma_{2}(c)=c ; \sigma_{3}(a)=b, \sigma_{3}(b)=a, \sigma_{3}(c)=c$.

Analogamente ao caso anterior, mostramos que $\operatorname{Stab}(\lambda)=G R\left\{\sigma_{1}, \sigma_{2}, \sigma_{3} \mid \sigma_{i}^{2}=\right.$ $\left.1, \sigma_{i} \sigma_{i+1} \sigma_{i}=\sigma_{i+1} \sigma_{i} \sigma_{i+1}, \sigma_{i} \sigma_{j}=\sigma_{j} \sigma_{i}, j \neq i \pm 1, i=1,2,3\right\}=S_{4}$. 
4. Sejam $a, b, c$ geradores do loop de código $L=C_{4}^{3}$ tal que $\lambda=\lambda(L)=(110000)$. Como existem 21 códigos diferentes em $O_{4}$, teremos 8 automorfismos que fixam $\lambda$. Para mostrar que Out $L \simeq D_{8}$, devemos primeiramente encontrar dois geradores, $\varphi$ e $\sigma, \varphi$ de ordem 4 e $\sigma$ de ordem 2, que fixem $\lambda$, e tais que $\sigma \varphi \sigma=\varphi^{3}$. Considere $\varphi \in G L_{3}(2)$ e $\sigma \in G L_{3}(2)$ tais que $\varphi(a)=b, \varphi(b)=b c, \varphi(c)=a b ; \sigma(a)=b$, $\sigma(b)=a$ e $\sigma(c)=c$. Temos que $\varphi$ e $\sigma$ fixam $\lambda$. Além disso, $\varphi$ tem ordem $4, \sigma$ tem ordem 2 e valem as relações $\sigma \varphi^{3}=\varphi \sigma, \varphi^{2} \sigma=\sigma \varphi^{2}$ e $\varphi^{3} \sigma=\sigma \varphi$.

Portanto, Out $C_{4}^{3} \simeq D_{8}=G R\left\{\sigma, \varphi \mid \varphi^{4}=1, \sigma^{2}=1, \sigma \varphi \sigma^{-1}=\varphi^{-1}\right\}=\operatorname{Stab}(\lambda)$.

5. Sejam $a, b, c$ geradores do loop de código $L=C_{5}^{3}$ tal que $\lambda=\lambda(L)=(100000)$. Como temos 28 códigos diferentes na órbita $O_{5}$, temos que encontrar 6 automorfismos que fixam $\lambda$.

Sejam $\varphi \in G L_{3}(2)$ e $\sigma \in G L_{3}(2)$ tais que $\varphi(a)=a, \varphi(b)=c, \varphi(c)=b ; \sigma(a)=a c$, $\sigma(b)=b c$ e $\sigma(c)=c$.

Temos que $\varphi$ e $\sigma$ fixam $\lambda, \sigma^{2}=\varphi^{2}=1$ e $\varphi \sigma \varphi=\sigma \varphi \sigma$.

Portanto, Out $C_{5}^{3} \simeq S_{3}=G R\{\varphi, \sigma \mid \varphi \sigma \varphi=\sigma \varphi \sigma\}=\operatorname{Stab}(\lambda)$.

\subsection{Loops de Código de posto 4}

Lema 2.2.1. Seja $V$ um 3-espaço não-trivial de dimensão 4. Então $\operatorname{dim} N(V)=1$ e $V$ tem uma base $\{a, b, c, d\}$ tal que $(a, b, c)=1$ e $N(V)=\mathbf{F}_{2} d=\{0, d\}$.

Demonstração. Como $V$ é um 3-espaço não-trivial, seja $(v, w, u)=1$, para alguns $v, w, u \in V$. Então existe $t \in V$ tal que $(v, w, t)=0$ e $\{v, w, u, t\}$ é uma base de $V$. Se $(v, w, t)=1$, então $(v, w, u+t)=(v, w, u)+(v, w, t)=0$, logo podemos supor $(v, w, t)=0$. 
Se $(v, u, t)=1$, então $(v, w, t+w)=(v, u, t+w)=0$ e podemos supor que $(v, u, t)=0$. Se $(w, u, t)=1$, então $t+v \in N(V)$, logo podemos $\operatorname{supor}(w, u, t)=0$.

Portanto, encontramos uma base $\{v, w, u, t\}$ tal que $(v, w, u)=1,(v, w, t)=$ $(v, u, t)=(w, u, t)=0$.

Se $X=\{a, b, c, d\}$ é uma base de um loop de código não associativo $L$ de posto 4 tal que $N(L)=F_{2} d$, então temos somente um associador não-trivial $(a, b, c)=-1$. Portanto, podemos reduzir um vetor característico de $L$ à $\lambda_{X}=\left(\lambda_{1}, \ldots, \lambda_{10}\right)$, onde

$$
\begin{gathered}
a^{2}=(-1)^{\lambda_{1}}, b^{2}=(-1)^{\lambda_{2}}, c^{2}=(-1)^{\lambda_{3}}, d^{2}=(-1)^{\lambda_{4}}, \\
{[a, b]=(-1)^{\lambda_{5}},[a, c]=(-1)^{\lambda_{6}},[a, d]=(-1)^{\lambda_{7}},} \\
{[b, c]=(-1)^{\lambda_{8}},[b, d]=(-1)^{\lambda_{9}},[c, d]=(-1)^{\lambda_{10}} .}
\end{gathered}
$$

Denotemos por $C_{1}^{4}, \ldots, C_{16}^{4}$ os loops de código com os seguintes vetores característicos correspondentes:

\begin{tabular}{l|l}
$L$ & $\lambda(L)$ \\
\hline$C_{1}^{4}$ & $(1110110100)$ \\
$C_{2}^{4}$ & $(0000000000)$ \\
$C_{3}^{4}$ & $(0000110100)$ \\
$C_{4}^{4}$ & $(0010100000)$ \\
$C_{5}^{4}$ & $(0000010100)$ \\
$C_{6}^{4}$ & $(1111110100)$ \\
$C_{7}^{4}$ & $(0001000000)$ \\
$C_{8}^{4}$ & $(0000001000)$
\end{tabular}

\begin{tabular}{l|l}
$L$ & $\lambda(L)$ \\
\hline$C_{9}^{4}$ & $(0100001000)$ \\
$C_{10}^{4}$ & $(0001111000)$ \\
$C_{11}^{4}$ & $(0001001000)$ \\
$C_{12}^{4}$ & $(0000001100)$ \\
$C_{13}^{4}$ & $(0110111100)$ \\
$C_{14}^{4}$ & $(0001001100)$ \\
$C_{15}^{4}$ & $(1001001100)$ \\
$C_{16}^{4}$ & $(0001111100)$
\end{tabular}

Tabela 2.3: Vetores característicos dos Loops de Código de posto 4

Teorema 2.2.1. Qualquer loop de código não associativo de posto 4 é isomorfo a um dos seguintes loops: $\left\{C_{1}^{4}, \ldots, C_{16}^{4}\right\}$. Além disso, todos esses loops são não isomorfos uns aos outros. 
Demonstração. Seja $L$ um loop de código de posto 4 com geradores $a, b, c, d$. Pelo lema 2.2.1, podemos supor $(d, L, L)=1$. Seja $C=C(d)$ o centralizador de $d$, ou seja, $C=\{v \in L \mid[v, d]=1\}$.

Um subloop $L_{0}$ de $L$ é um subloop característico se, e somente se, para todo $\varphi \in \operatorname{Aut}(L), L_{0}^{\varphi}=L_{0}$. Então, claramente, temos que $C$ é um subloop característico de $L$.

(I) Se $C=L$, então temos duas possibilidades:

1. $d^{2}=1$

Neste caso, $L \simeq \mathbb{Z}_{2} \times L_{1}$, onde $L_{1}$ é um loop de código não-associativo de posto 3. Como existem 5 loops de código não associativos de posto 3 , logo obtemos 5 loops de código não isomorfos de posto 4, denotados por $C_{i}^{4}, i=1, \ldots, 5$, ou seja,

$$
C_{i}^{4} \cong Z_{2} \times C_{i}^{3}, \quad i=1, \ldots, 5
$$

2. $d^{2}=-1$

Seja $\langle d\rangle=\{1,-1, d,-d\}$ o grupo gerado por $d$. Sabemos que $\langle d\rangle \cong Z_{4}$ e que $Z_{4}$ é loop de código. Novamente, seja $L_{1}$ um loop de código não-associativo de posto 3. Temos que $Z_{4} * L_{1}$ é um loop de código não-associativo de posto 4. Neste caso, $L \simeq Z_{4} * L_{1}$. Mas, pelo Lema 1.3.4, é possível provar que $Z_{4} * C_{1}^{3} \cong Z_{4} * C_{3}^{3}$ e $Z_{4} * C_{2}^{3} \cong Z_{4} * C_{4}^{3} \cong Z_{4} * C_{5}^{3}$. Para isto, basta mostrarmos que $C_{1}^{3}$ e $C_{3}^{3}$ são isomorfos como 2-espaços, assim como $C_{2}^{3}, C_{4}^{3}$ e $C_{5}^{3}$. De fato, como um vetor característico do 2-espaço correspondente a um dado loop de código $L$ de posto $n$ pode ser obtido a partir de um vetor característico $\lambda(L)$ omitindo as primeiras $n$ coordenadas de $\lambda(L)$, então apenas comparando os vetores característicos obtemos o que queremos. 
Portanto, temos somente dois novos loops:

$$
C_{6}^{4}=Z_{4} * C_{1}^{3} \quad \text { e } \quad C_{7}^{4}=Z_{4} * C_{2}^{3}
$$

(II) Se $C \neq L$, então $C$ é um grupo de código de posto 3. De fato, suponhamos que para todo $u \neq d \in X=\{a, b, c, d\}$, o conjunto de geradores de $L$, temos $[u, d]=-1$, mas como $[a b, d]=[a c, d]=1$, logo temos que $\{d, a b, a c\}$ gera $C$. Agora, se supormos que, por exemplo, $[a, d]=1,[b, d]=[c, d]=-1$, teremos $[b c, d]=1, \log 0\{d, a, b c\}$ gera $C$.

Existem 5 grupos de códigos não isomorfos de posto 3:

1. $G_{1}^{3}=Z_{2}^{4}$

2. $G_{2}^{3}=Z_{4} \times Z_{2}^{2}$;

3. $G_{3}^{3}=D_{8} \times Z_{2}$;

4. $G_{4}^{3}=Q \times Z_{2}$, onde $Q$ é o grupo dos quatérnios;

5. $G_{5}^{3}=D_{8} * Z_{4}$, onde $D_{8}$ é o grupo diedral de 8 elementos.

Faremos uma análise de cada caso:

1. $C=G_{1}^{3}$

Neste caso, $b, c, d \in C$, então $b^{2}=c^{2}=d^{2}=[b, c]=[b, d]=[c, d]=1 \mathrm{e}$ $[a, d]=-1$.

Se $a^{2}=-1$, então $(a d)^{2}=a^{2} d^{2}[a, d]=1$. Logo, podemos supor $a^{2}=1$.

Denotemos este loop por $C_{8}^{4}$.

2. $C=G_{2}^{3}$. Temos 2 casos:

(a) $d^{2}=1$.

Aqui, $b^{2}=-1, c^{2}=1,[a, d]=-1,[a, b]=[a, c]=1$. Como no caso anterior, podemos supor $a^{2}=1$.

Denotemos este loop por $C_{9}^{4}$. 
(b) $d^{2}=-1$.

Temos $b^{2}=c^{2}=1,[a, d]=-1$.

Se $[a, b]=[a, c]=-1$, então $[a, b c]=-1,(a b)^{2}=-a^{2}$ e daí, podemos supor $a^{2}=1$. Denotemos este loop por $C_{10}^{4}$.

Se $[a, b]=1$, então sem perda de generalidade, $[a, c]=1$, desde que $[a, b c]=-[a, c]$. Como $(a c)^{2}=-a^{2}$, podemos supor $a^{2}=1$. Denotemos este loop por $C_{11}^{4}$.

3. $C=G_{3}^{3}$

Neste caso, $d^{2}=1, c^{2}=b^{2}=1,[b, c]=-1,[a, d]=-1$.

Como $[a, b d]=-[a, b],(a d)^{2}=-a^{2}$, podemos supor $[a, b]=[a, c]=1 \mathrm{e}$ $a^{2}=1$. Denotemos este loop por $C_{12}^{4}$.

4. $C=G_{4}^{3}$

Neste caso, $d^{2}=1, a^{2}=1, b^{2}=c^{2}=[b, c]=-1,[a, d]=-1$. Existem 2 possibilidades:

(a) $[a, b]=[a, c]=-1$

Denotemos este loop por $C_{13}^{4}$.

(b) $[a, b]=[a, c]=1$

Este loop é isomorfo a $C_{13}^{4}$, visto que podemos substituir $b$ por $b c$ e $c$ por $c d$, pois $(b c)^{2}=(c d)^{2}=[b c, c d]=-1,[a, b c]=[a, c d]=-1$.

5. $C=G_{5}^{3}$

Neste caso, $d^{2}=-1, a^{2}=1, b^{2}=c^{2}=1,[b, c]=-1,[a, d]=-1$. Existem 3 possibilidades:

(a) $[a, b]=[a, c]=1,[a, b c]=-1$. Neste caso, temos dois loops não isomorfos:

Caso $a^{2}=1$ : Denotamos este loop por $C_{14}^{4}$.

Caso $a^{2}=-1$ : Denotamos este loop por $C_{15}^{4}$. 
(b) $[a, b]=[a, c]=-1,[a, b c]=-1$. Neste caso, $(a b d)^{2}=-a^{2}$ e $[a b d, b]=$ $[a b d, c]=-1$. Portanto, podemos supor $a^{2}=1$. Denotamos este loop por $C_{16}^{4}$.

(c) $[a, b]=1,[a, c]=-1,[a, b c]=1$. Este loop é isomorfo ao loop $C_{16}^{4}$, visto que podemos substituir $b$ por $b c d$.

Corolário 2.2.1. Existem 5 classes de $Z_{4}$-equivalência de loops de código de posto 4:

$$
\begin{aligned}
Z_{1}^{4} & =\left\{C_{1}^{4}, C_{3}^{4}, C_{4}^{4}, C_{6}^{4}\right\}, \\
Z_{2}^{4} & =\left\{C_{5}^{4}, C_{8}^{4}, C_{9}^{4}, C_{10}^{4}, C_{11}^{4}\right\}, \\
Z_{3}^{4} & =\left\{C_{12}^{4}, C_{14}^{4}, C_{15}^{4}\right\}, \\
Z_{4}^{4} & =\left\{C_{2}^{4}, C_{7}^{4}\right\} \\
Z_{5}^{4} & =\left\{C_{13}^{4}, C_{16}^{4}\right\},
\end{aligned}
$$

Demonstração. Pelo Lema 1.3.4, temos que provar que $C_{i}^{4} \cong C_{j}^{4}$ como 2-espaços se, e somente se, $i, j \in Z_{k}^{4}$ para alguns $k=1, \ldots, 4$. Escreveremos $i \sim j$ ao invés de $C_{i}^{4} \sim Z_{4} C_{j}^{4}$.

Um vetor característico do 2-espaço correspondente a um dado loop de código $L$ de posto $n$ pode ser obtido a partir de um vetor característico $\lambda(L)$ omitindo as primeiras $n$ coordenadas de $\lambda(L)$.

Portanto, pela Tabela 2.3 e pelo Teorema 2.2.1, temos $1 \sim 3 \sim 6,2 \sim 7,8 \sim 9 \sim 11$, $12 \sim 14 \sim 15$ e $13 \sim 16$.

Seja $X=\{a, b, c, d\}$ um conjunto de geradores de $C_{3}^{4}$ tal que $(a, b, c)=[a, b]=$ $[a, c]=[b, c]=-1$, mas então $[a, a b c]=[b, a b c]=1 \mathrm{e}$, para um conjunto de geradores $Y=\{a, b, a b c, d\}$, temos um vetor característico do 2-espaço $C_{3}^{4}$ da forma: $\lambda_{Y}\left(C_{3}^{4}\right)=(100000)=\lambda\left(C_{4}^{4}\right)$. Portanto, $3 \sim 4$. 
Seja $X=\{a, b, c, d\}$ um conjunto de geradores de $C_{5}^{4}$ tal que $(a, b, c)=[a, c]=$ $[a, d]=-1$, mas então $[a, c d]=1$ e para um conjunto de geradores $Y=\{a, b, c d, d\}$ temos um vetor característico do 2-espaço $C_{5}^{4}$ da forma: $\lambda_{Y}\left(C_{5}^{4}\right)=(001000)=\lambda\left(C_{8}^{4}\right)$. Portanto, $5 \sim 8$.

Seja $X=\{a, b, c, d\}$ um conjunto de geradores de $C_{10}^{4}$ tal que $(a, b, c)=[a, b]=$ $[a, c]=[a, d]=-1$, mas então $[a, b d]=[a, c d]=1 \mathrm{e}$, para um conjunto de geradores $Y=\{a, b d, c d, d\}$, temos um vetor característico do 2-espaço $C_{10}^{4}$ da forma: $\lambda_{Y}\left(C_{10}^{4}\right)=(001000)=\lambda\left(C_{8}^{4}\right)$. Portanto, $10 \sim 8$.

Agora, temos que provar que todas essas classes $Z_{1}^{4}, \ldots, Z_{5}^{4}$ são não-equivalentes. É claro que um espaço $C(N(L))=\{x \in L \mid[x, N(L)]=1\}$ é um espaço característico de $L$. Em nosso caso, $N(L)=\mathbf{F}_{2} d$ e para $C=C(d)$ temos três possibilidades:

1. $C=L$,

2. $[C, C]=(C, C, C)=1$,

3. $C \neq L,[C, C] \neq 1$.

Além disso, temos a segunda possibilidade somente para $Z_{3}^{4}$ e a terceira possibilidade somente para $Z_{4}^{4}$. Para provar que $1 \not 2$ notamos que $N_{2}\left(C_{1}^{4}\right) \simeq N_{2}\left(C_{2}^{4}\right) \simeq \mathbf{F}_{2} d$, mas os 2-espaços $C_{1}^{4} / N_{2}\left(C_{1}^{4}\right)$ e $C_{2}^{4} / N_{2}\left(C_{2}^{4}\right)$ são não isomorfos. Analogamente, prova-se que $1 \not 5$ e $2 \not 5$.

\subsection{Automorfismos dos Loops de Código de posto}

4

Sejam $(a, b, c, d)$ uma base para $C_{i}^{4}$ e $\sigma \in G L_{4}(2)$ um automorfismo de $C_{i}^{4}$. Para cada $\sigma$ encontramos uma nova base de $C_{i}^{4}$, denotemos esta base por $(u, v, w, d)$, ou seja, $\left(a^{\sigma}=u, b^{\sigma}=v, c^{\sigma}=w, d^{\sigma}=d\right)$. Analogamente ao estudo das órbitas dos loops de código de posto 3, Proposição 2.1.1, podemos exibir todos os códigos de cada órbita 
associada a um loop de código de posto 4. De fato, basta fixar um vetor característico representante de uma certa órbita, percorrer $G L_{4}(2)$ e encontrar todas as bases possíveis $(u, v, w, d)$. Assim, a partir dos quadrados de cada gerador e dos comutadores de dois a dois dos geradores obtemos um vetor característico pertencente a órbita do vetor característico representante fixado.

Como ilustração, encontraremos a órbita do loop $C_{3}^{4}$. Neste caso, $a^{2}=b^{2}=c^{2}=$ $d^{2}=1,[a, b]=[a, c]=[b, c]=-1$ e $[a, d]=[b, d]=[c, d]=1$. Se $\sigma \in G L_{4}(2)$, então temos 7 possibilidades para $(u, v, w, d):(a, b, c, d),(a, b, a c, d),(a, a b, c, d),(a, a b, a c, d)$, $(a b, a, c, d),(a b, a, a c, d),(a c, a b, a, d)$, cujos vetores característicos associados são dados respectivamente por: (0000110100), (0010110100), (0100110100), (0110110100), (1000110100), (1010110100), (1100110100). Estes vetores estão na órbita de $C_{3}^{4}$. Logo, $\left|O_{3}^{4}\right|=7$.

Procedendo de maneira análoga a ilustração, obtemos $\left|O_{1}^{4}\right|=1,\left|O_{2}^{4}\right|=7,\left|O_{4}^{4}\right|=21$, $\left|O_{5}^{4}\right|=\left|O_{14}^{4}\right|=\left|O_{15}^{4}\right|=28,\left|O_{6}^{4}\right|=8,\left|O_{7}^{4}\right|=\left|O_{8}^{4}\right|=\left|O_{13}^{4}\right|=56,\left|O_{9}^{4}\right|=\left|O_{12}^{4}\right|=\left|O_{16}^{4}\right|=$ $168,\left|O_{10}^{4}\right|=\left|O_{11}^{4}\right|=112$.

Para encontrar todos esses valores e os elementos de cada órbita, precisamos calcular todos os quadrados e comutadores de $u, v, w$ e $d$. Pensando nisto, as Tabelas $A, B, C$ e $D$ do Apêndice A mostram todos os valores necessários de quadrados e comutadores. Cada coluna da tabela se refere a um loop de código.

Quando necessário denotaremos os vetores característicos $\lambda\left(C_{i}^{k}\right)$ por $\lambda_{i}^{k}$, onde $k$ representa o posto do loop de código. Denotamos por $\operatorname{Stab}\left(\lambda_{i}^{k}\right)$ o grupo de automorfismos de $G L_{k}(2)$ que fixam $\lambda_{i}^{k}$.

Para provarmos a próxima proposição precisamos de alguns resultados da teoria de grupos, sobre produto direto de grupos, enunciados a seguir. As demonstrações são encontradas em [12].

Teorema 2.3.1. Sejam $G, G_{1}, \ldots, G_{n}$ grupos. Então o grupo $G$ é isomorfo ao grupo 
$G_{1} \times \cdots \times G_{n}$ se, e somente se, $G$ possui subgrupos $H_{1} \cong G_{1}, \ldots, H_{n} \cong G_{n}$ tais que:

1) $G=H_{1} \ldots H_{n}$

2) $H_{i} \triangleleft G, \forall i=1, \ldots, n$.

3) $H_{i} \cap\left(H_{1} \ldots H_{i-1} H_{i+1} \ldots H_{n}\right)=\{e\}, \forall i=1, \ldots, n$.

Lema 2.3.1. Sejam $G$ um grupo e $H_{1}, \ldots, H_{n}$ subgrupos de $G$. Então as condições 1),2),3) do Teorema 2.3.1 são satisfeitas se, e somente se, as condições 4) e 5) seguintes são satisteitas:

4) Para cada $g \in G$, existem elementos univocamente determinados $x_{1} \in H_{1}, \ldots, x_{n} \in H_{n}$ tais que $g=x_{1} \ldots x_{n}$.

5) Para cada $i \neq j$, temos $x y=y x$, para cada $x \in H_{i}$ e para cada $y \in H_{j}$.

Teorema 2.3.2. Sejam $G, H, K$ grupos. Então $G$ é isomorfo ao produto semidireto de $H$ e $K$, denotado por $H \rtimes K$ se, e somente se, $G$ possui subgrupos $H_{1} \simeq H$ e $K_{1} \simeq K$ tais que:

1. $G=K_{1} H_{1}$.

2. $H_{1} \triangleleft G$.

3. $K_{1} \cap H_{1}=\{e\}$.

Proposição 2.3.1. Na notação acima, temos:

1. $\operatorname{Out} C_{1}^{4} \simeq \mathbb{Z}_{2}^{3} \rtimes G L_{3}(2)$;

2. $\mathrm{Out} C_{2}^{4} \simeq \mathbb{Z}_{2}^{3} \rtimes S_{4}$;

3. $\mathrm{Out} C_{3}^{4} \simeq \mathbb{Z}_{2}^{3} \rtimes S_{4}$;

4. $\mathrm{OutC}_{4}^{4} \simeq \mathbb{Z}_{2}^{3} \rtimes D_{8}$, 
5. Out $C_{5}^{4} \simeq S_{4} \times \mathbb{Z}_{2}$;

6. $\mathrm{OutC}_{6}^{4} \simeq G L_{3}(2)$,

7. $\operatorname{Out} C_{7}^{4} \simeq S_{4}$,

8. $\mathrm{Out} C_{8}^{4} \simeq S_{4}$

9. $\mathrm{Out} C_{9}^{4} \simeq D_{8}$,

10. Out $C_{10}^{4} \simeq S_{3} \times \mathbb{Z}_{2}$,

11. Out $C_{11}^{4} \simeq S_{3} \times \mathbb{Z}_{2}$,

12. $\mathrm{Out} C_{12}^{4} \simeq D_{8}$

13. $\mathrm{Out} C_{13}^{4} \simeq S_{4}$;

14. Out $C_{14}^{4} \simeq \mathbb{Z}_{2}^{3} \rtimes S_{3} ;$

15. Out $C_{15}^{4} \simeq \mathbb{Z}_{2}^{3} \rtimes S_{3} ;$

16. Out $C_{16}^{4} \simeq \mathbb{Z}_{2}^{3}$.

Demonstração. 1. Sendo $(a, b, c, d)$ uma base do loop $C_{1}^{4}$ com vetor característico $\lambda_{1}^{4}=(1110110100)$, temos $a^{2}=b^{2}=c^{2}=d^{2}=-1$ e $[a, b]=[a, c]=[a, d]=$ $[b, c]=[b, d]=[c, d]=-1$. Também temos que $(a, b, c)$ é uma base do loop de código de posto 3 com vetor característico $\lambda_{1}^{3}=(111111)$.

Seja $\varphi \in \operatorname{Stab}\left(\lambda_{1}^{3}\right)$. Estendemos $\varphi$ a $\tilde{\varphi}$ definindo:

$$
\begin{aligned}
& \tilde{\varphi}: a \quad a^{\varphi} d^{\alpha_{1}}=u \\
& b \longmapsto b^{\varphi} d^{\alpha_{2}}=v \\
& \text { c } \quad c^{\varphi} d^{\alpha_{3}}=w \quad, \quad \alpha_{i} \in\{0,1\}, i=1,2,3 . \\
& \text { d } \quad d
\end{aligned}
$$


A matriz que representa $\tilde{\varphi}$ é da forma

$$
\tilde{\varphi}=\left(\begin{array}{lll|l} 
& & & \alpha_{1} \\
& \varphi & & \alpha_{2} \\
& & \alpha_{3} \\
\hline 0 & 0 & 0 & 1
\end{array}\right) .
$$

Claramente $\tilde{\varphi} \in G L_{4}(2)$, resta provar que $\tilde{\varphi} \in \operatorname{Stab}\left(\lambda_{1}^{4}\right)$, ou seja, que $\left(\lambda_{1}^{4}\right)^{\tilde{\varphi}}=\lambda_{1}^{4}$.

Para isto, devemos encontrar para quais $\left(\alpha_{1}, \alpha_{2}, \alpha_{3}\right)$ temos as relações abaixo:

(a) $u^{2}=v^{2}=w^{2}=1$

(b) $[u, v]=[u, w]=[v, w]=-1$ e $[u, d]=[v, d]=[w, d]=1$

Vejamos:

(a) Caso $\alpha_{i}=0$ : $\left(a^{\varphi}\right)^{2}=\left(b^{\varphi}\right)^{2}=\left(c^{\varphi}\right)^{2}=-1$, já que $\varphi \in \operatorname{Stab}\left(\lambda_{1}^{3}\right)$.

Caso $\alpha_{i}=1:\left(a^{\varphi} d\right)^{2}=\left(a^{\varphi}\right)^{2} d^{2}\left[a^{\varphi}, d\right]=-1$, pois $\left(a^{\varphi}\right)^{2}=-1\left(\varphi \in \operatorname{Stab}\left(\lambda_{1}^{3}\right)\right)$ e $d^{2}=\left[a^{\varphi}, d\right]=1([a, d]=[b, d]=[c, d]=1)$.

(b) Claramente temos $[u, d]=[v, d]=[w, d]=1$.

Agora vejamos que $[u, v]=[u, w]=[v, w]=-1$ :

Caso $\alpha_{i}=0, \operatorname{temos}\left[a^{\varphi}, b^{\varphi}\right]=-1$, pois $\varphi \in \operatorname{Stab}\left(\lambda_{1}^{3}\right)$.

Caso $\alpha_{i}=1$, temos $\left[a^{\varphi} d, b^{\varphi} d\right]=\left[a^{\varphi}, b^{\varphi} d\right]\left[d, b^{\varphi} d\right]\left(a^{\varphi}, b^{\varphi}, d\right)$, mas $\left[d, b^{\varphi} d\right]=$ $\left(a^{\varphi}, b^{\varphi}, d\right)=1$, então $\left[a^{\varphi} d, b^{\varphi} d\right]=\left[a^{\varphi}, b^{\varphi} d\right]=\left[a^{\varphi}, b^{\varphi}\right]\left[a^{\varphi}, d\right]=\left[a^{\varphi}, b^{\varphi}\right]=-1$.

Logo, $[u, v]=-1$. Analogamente se provam os outros dois casos.

Portanto, para todo $\varphi \in \operatorname{Stab}\left(\lambda_{1}^{3}\right)$ e para quaisquer valores de $\left(\alpha_{1}, \alpha_{2}, \alpha_{3}\right)$, temos $\tilde{\varphi} \in \operatorname{Stab}\left(\lambda_{1}^{4}\right)$. Pela Proposição 2.1.2 (grupos dos automorfismos dos loops de posto 3), temos $\left|\operatorname{Stab}\left(\lambda_{1}^{3}\right)\right|=168$. Logo $\left|\operatorname{Stab}\left(\lambda_{1}^{4}\right)\right|=168.8=1344$. 
Consideremos os seguintes estabilizadores de $\lambda_{1}^{4}$ :

$$
\begin{aligned}
& \sigma_{1}=\left(\begin{array}{cccc}
1 & 0 & 0 & 0 \\
1 & 1 & 0 & 0 \\
0 & 0 & 1 & 0 \\
0 & 0 & 0 & 1
\end{array}\right) \quad \sigma_{2}=\left(\begin{array}{cccc}
1 & 0 & 0 & 0 \\
0 & 1 & 0 & 0 \\
1 & 0 & 1 & 0 \\
0 & 0 & 0 & 1
\end{array}\right) \quad \sigma_{3}=\left(\begin{array}{cccc}
1 & 0 & 0 & 0 \\
0 & 1 & 0 & 0 \\
0 & 0 & 1 & 1 \\
0 & 0 & 0 & 1
\end{array}\right) \\
& \sigma_{4}=\left(\begin{array}{cccc}
1 & 0 & 0 & 0 \\
0 & 1 & 1 & 0 \\
0 & 0 & 1 & 0 \\
0 & 0 & 0 & 1
\end{array}\right) \quad \sigma_{5}=\left(\begin{array}{cccc}
1 & 1 & 0 & 0 \\
0 & 1 & 0 & 0 \\
0 & 0 & 1 & 0 \\
0 & 0 & 0 & 1
\end{array}\right) \quad \sigma_{6}=\left(\begin{array}{cccc}
1 & 0 & 1 & 0 \\
0 & 1 & 0 & 0 \\
0 & 0 & 1 & 0 \\
0 & 0 & 0 & 1
\end{array}\right) \\
& \rho_{1}=\left(\begin{array}{cccc}
1 & 0 & 0 & 0 \\
0 & 1 & 0 & 1 \\
0 & 0 & 1 & 0 \\
0 & 0 & 0 & 1
\end{array}\right) \quad \rho_{2}=\left(\begin{array}{cccc}
1 & 0 & 0 & 0 \\
0 & 1 & 0 & 1 \\
0 & 0 & 1 & 1 \\
0 & 0 & 0 & 1
\end{array}\right) \quad \rho_{3}=\left(\begin{array}{cccc}
1 & 0 & 0 & 1 \\
0 & 1 & 0 & 0 \\
0 & 0 & 1 & 0 \\
0 & 0 & 0 & 1
\end{array}\right)
\end{aligned}
$$

Temos $\sigma_{i}^{2}=2, i=1, \ldots, 6, \rho_{i}^{2}=1, \rho_{i} \rho_{j}=\rho_{j} \rho_{i}, i, j=1,2,3 . \quad$ Sejam $H=G R\left\{\sigma_{i}, i=1, \ldots, 6\right\}$ e $N=G R\left\{\rho_{i}, i=1,2,3 \mid \rho_{i}^{2}=1, \rho_{i} \rho_{j}=\rho_{j} \rho_{i}\right\}$, subgrupos de $\operatorname{Stab}\left(\lambda_{1}^{4}\right)$. Sabemos que $H \simeq G L_{3}(2), N \simeq \mathbb{Z}_{2}^{3}$, e ainda temos $\operatorname{Stab}\left(\lambda_{1}^{4}\right)=H N$ e $H \cap N=\{1\}$. Além disso, com cálculos diretos se mostra que $N$ é normal em $\operatorname{Stab}\left(\lambda_{1}^{4}\right)$. Portanto, $\operatorname{Out}\left(C_{1}^{4}\right) \simeq \mathbb{Z}_{2}^{3} \rtimes G L_{3}(2)$.

2. Neste caso $\lambda_{2}^{4}=(0000000000)$, ou seja, $a^{2}=b^{2}=c^{2}=d^{2}=1$ e $[a, b]=[a, c]=$ $[a, d]=[b, c]=[b, d]=[c, d]=1$. A base $(a, b, c)$ nos fornece o loop de código de posto 3 , com $\lambda_{2}^{3}=(000000)$.

Seja $\varphi \in \operatorname{Stab}\left(\lambda_{2}^{3}\right)$. Analogamente ao caso anterior, estendemos $\varphi$ para 
$\tilde{\varphi} \in G L_{4}(2)$ por:

$$
\begin{aligned}
& \tilde{\varphi}: a \quad a^{\varphi} d^{\alpha_{1}} \\
& b \longmapsto b^{\varphi} d^{\alpha_{2}} \\
& \text { c } \quad c^{\varphi} d^{\alpha_{3}} \\
& , \quad \alpha_{i} \in\{0,1\}, i=1,2,3 . \\
& \text { d } \quad d
\end{aligned}
$$

Devemos provar que $\tilde{\varphi} \in \operatorname{Stab}\left(\lambda_{2}^{4}\right)$, ou seja, que $\left(\lambda_{2}^{4}\right)^{\tilde{\varphi}}=\lambda_{2}^{4}$. De fato, como $\varphi \in \operatorname{Stab}\left(\lambda_{2}^{3}\right)$, então

(a) $\left(a^{\varphi} d^{\alpha_{1}}\right)^{2}=\left(a^{\varphi}\right)^{2}\left(d^{\alpha_{1}}\right)^{2}\left[a^{\varphi}, d^{\alpha_{1}}\right]=1$.

Analogamente, temos $\left(b^{\varphi} d^{\alpha_{2}}\right)^{2}=\left(c^{\varphi} d^{\alpha_{3}}\right)^{2}=1$.

(b) Claramente $\left[a^{\varphi} d^{\alpha_{1}}, d\right]=\left[b^{\varphi} d^{\alpha_{2}}, d\right]=\left[c^{\varphi} d^{\alpha_{3}}, d\right]=1$.

Vejamos que $\left[a^{\varphi} d^{\alpha_{1}}, b^{\varphi} d^{\alpha_{2}}\right]=1$ :

$$
\left[a^{\varphi} d^{\alpha_{1}}, b^{\varphi} d^{\alpha_{2}}\right]=\left[a^{\varphi}, b^{\varphi} d^{\alpha_{2}}\right]\left[d^{\alpha_{1}}, b^{\varphi} d^{\alpha_{2}}\right]\left(a^{\varphi}, d^{\alpha_{1}}, b^{\varphi} d^{\alpha_{2}}\right)
$$

$\operatorname{Temos}\left[d^{\alpha_{1}}, b^{\varphi} d^{\alpha_{2}}\right]=\left(a^{\varphi}, d^{\alpha_{1}}, b^{\varphi} d^{\alpha_{2}}\right)=1$, então

$$
\left[a^{\varphi} d^{\alpha_{1}}, b^{\varphi} d^{\alpha_{2}}\right]=\left[a^{\varphi}, b^{\varphi} d^{\alpha_{2}}\right]=\left[a^{\varphi}, b^{\varphi}\right]\left[a^{\varphi}, d^{\alpha_{2}}\right]\left(a^{\varphi}, b^{\varphi}, d^{\alpha_{2}}\right) .
$$

Como $\left[a^{\varphi}, b^{\varphi}\right]=\left[a^{\varphi}, d^{\alpha_{2}}\right]=\left(a^{\varphi}, b^{\varphi}, d^{\alpha_{2}}\right)=1$, portanto $\left[a^{\varphi} d^{\alpha_{1}}, b^{\varphi} d^{\alpha_{2}}\right]=1$.

Analogamente, temos $\left[a^{\varphi} d^{\alpha_{1}}, c^{\varphi} d^{\alpha_{3}}\right]=\left[b^{\varphi} d^{\alpha_{2}}, c^{\varphi} d^{\alpha_{3}}\right]=1$.

Provamos que, para qualquer $\varphi \in \operatorname{Stab}\left(\lambda_{2}^{3}\right)$ e quaisquer valores de $\left(\alpha_{1}, \alpha_{2}, \alpha_{3}\right)$, $\tilde{\varphi} \in \operatorname{Stab}\left(\lambda_{2}^{4}\right)$.

Pela Proposição 2.1.2 (grupos dos automorfismos dos loops de posto 3), temos $\left|\operatorname{Stab}\left(\lambda_{2}^{3}\right)\right|=24$. Logo, $\left|\operatorname{Stab}\left(\lambda_{2}^{4}\right)\right|=24.8=192$.

Para provar que Out $C_{2}^{4} \simeq \mathbb{Z}_{2}^{3} \rtimes S_{4}$, basta considerar os subgrupos $H$ e $N$ de 
$\operatorname{Stab}\left(\lambda_{2}^{4}\right)$ dados respectivamente por:

$$
H=G R\left\{\sigma_{i}, i=1,2,3 \mid \sigma_{i}^{2}=1, \sigma_{i} \sigma_{i+1} \sigma_{i}=\sigma_{i+1} \sigma_{i} \sigma_{i+1}, \sigma_{1} \sigma_{3}=\sigma_{3} \sigma_{1}\right\} \simeq S_{4}
$$

e

$$
N=G R\left\{\rho_{i}, i=1,2,3 \mid \rho_{i}^{2}=1, \rho_{i} \rho_{j}=\rho_{j} \rho_{i}\right\} \simeq \mathbb{Z}_{2}^{3}
$$

Neste caso, consideramos os geradores destes grupos dados da seguinte maneira:

$$
\begin{array}{ccc}
\sigma_{1}=\left(\begin{array}{cccc}
1 & 0 & 0 & 0 \\
0 & 0 & 1 & 0 \\
0 & 1 & 0 & 0 \\
0 & 0 & 0 & 1
\end{array}\right) & \sigma_{2}=\left(\begin{array}{cccc}
0 & 1 & 0 & 0 \\
1 & 0 & 0 & 0 \\
0 & 0 & 1 & 0 \\
0 & 0 & 0 & 1
\end{array}\right) & \sigma_{3}=\left(\begin{array}{cccc}
1 & 0 & 0 & 0 \\
1 & 1 & 0 & 0 \\
1 & 0 & 1 & 0 \\
0 & 0 & 0 & 1
\end{array}\right) \\
\rho_{1}=\left(\begin{array}{llll}
1 & 0 & 0 & 0 \\
0 & 1 & 0 & 1 \\
0 & 0 & 1 & 0 \\
0 & 0 & 0 & 1
\end{array}\right) & \rho_{2}=\left(\begin{array}{llll}
1 & 0 & 0 & 0 \\
0 & 1 & 0 & 1 \\
0 & 0 & 1 & 1 \\
0 & 0 & 0 & 1
\end{array}\right) & \rho_{3}=\left(\begin{array}{llll}
1 & 0 & 0 & 1 \\
0 & 1 & 0 & 0 \\
0 & 0 & 1 & 0 \\
0 & 0 & 0 & 1
\end{array}\right)
\end{array}
$$

Temos que $\operatorname{Stab}\left(\lambda_{2}^{4}\right)=H N, H \cap N=\{1\}$ e $N$ é subgrupo normal de $\operatorname{Stab}\left(\lambda_{2}^{4}\right)$.

Observação:

i) Em todos os casos seguintes consideraremos $\varphi \in \operatorname{Stab}\left(\lambda_{i}^{3}\right)$ e $\tilde{\varphi} \in G L_{4}(2)$ uma extensão de $\varphi$, definida como nos casos anteriores.

ii) Para qualquer vetor característico dos casos $3, \ldots, 7$ temos, para uma base fixada $(a, b, c, d)$, que $[a, d]=[b, d]=[c, d]=1, \operatorname{logo}\left[x d^{\alpha_{1}}, y d^{\alpha_{2}}\right]=[x, y]$ e $[x, d]=1$, para quaisquer $x, y \in\left\{a^{\varphi}, b^{\varphi}, c^{\varphi}\right\}$. Logo, nestes casos, não será necessário calcularmos os comutadores, visto que $\varphi \in \operatorname{Stab}\left(\lambda_{i}^{3}\right)$. Basta analisarmos os quadrados dos elementos $a^{\tilde{\varphi}}, b^{\tilde{\varphi}}, c^{\tilde{\varphi}}$. 
3. Neste caso $\lambda_{3}^{4}=(0000110100)$ e $\lambda_{3}^{3}=(000111)$. Temos

$$
\left(a^{\tilde{\varphi}}\right)^{2}=\left(a^{\varphi} d^{\alpha_{1}}\right)^{2}=\left(a^{\varphi}\right)^{2}\left(d^{\alpha_{1}}\right)^{2}\left[a^{\varphi}, d\right]=1 .
$$

Analogamente, temos $\left(b^{\tilde{\varphi}}\right)^{2}=\left(c^{\tilde{\varphi}}\right)^{2}=1$. Então, para qualquer tripla $\left(\alpha_{1}, \alpha_{2}, \alpha_{3}\right)$, temos $\tilde{\varphi} \in \operatorname{Stab}\left(\lambda_{3}^{4}\right)$. Além disso, sabemos que $\left|\operatorname{Stab}\left(\lambda_{3}^{3}\right)\right|=24, \operatorname{logo}$ $\left|\operatorname{Stab}\left(\lambda_{3}^{4}\right)\right|=24.8=192$.

Analogamente ao caso anterior, prova-se que Out $C_{2}^{4} \simeq \mathbb{Z}_{2}^{3} \rtimes S_{4}$. Neste caso, basta considerar os geradores dos grupos $H \simeq S_{4}$ e $N \simeq \mathbb{Z}_{2}^{3}$, respectivamente dados por:

$$
\begin{array}{ccc}
\sigma_{1}=\left(\begin{array}{cccc}
1 & 0 & 0 & 0 \\
0 & 0 & 1 & 0 \\
0 & 1 & 0 & 0 \\
0 & 0 & 0 & 1
\end{array}\right) & \sigma_{2}=\left(\begin{array}{cccc}
0 & 1 & 0 & 0 \\
1 & 0 & 0 & 0 \\
0 & 0 & 1 & 0 \\
0 & 0 & 0 & 1
\end{array}\right) & \sigma_{3}=\left(\begin{array}{llll}
1 & 1 & 1 & 0 \\
0 & 1 & 0 & 0 \\
0 & 0 & 1 & 0 \\
0 & 0 & 0 & 1
\end{array}\right) \\
\rho_{1}=\left(\begin{array}{llll}
1 & 0 & 0 & 0 \\
0 & 1 & 0 & 1 \\
0 & 0 & 1 & 0 \\
0 & 0 & 0 & 1
\end{array}\right) & \rho_{2}=\left(\begin{array}{llll}
1 & 0 & 0 & 0 \\
0 & 1 & 0 & 1 \\
0 & 0 & 1 & 1 \\
0 & 0 & 0 & 1
\end{array}\right) & \rho_{3}=\left(\begin{array}{llll}
1 & 0 & 0 & 1 \\
0 & 1 & 0 & 0 \\
0 & 0 & 1 & 0 \\
0 & 0 & 0 & 1
\end{array}\right)
\end{array}
$$

4. Sejam $\lambda_{4}^{4}=(0010100000)$ e $\lambda_{4^{\prime}}^{3}=(001100)$. Temos que $\left|\operatorname{Stab}\left(\lambda_{4^{\prime}}^{3}\right)\right|=8$ pois $\lambda_{4^{\prime}}^{3} \in O_{4}^{3}$. De fato, suponha $a^{\varphi}=x, b^{\varphi}=y, c^{\varphi}=z$. Então temos 8 possibilidades para $(x, y, z)$, dadas por $(a, b, c),(b, a, c),(a, b, a c),(b, a, a c),(a, b, b c),(b, a, b c)$, $(a, b, a b c),(b, a, a b c)$. Em qualquer caso $x^{2}=y^{2}=1, z^{2}=-1,[x, y]=-1$, $[x, y]=[y, z]=1 . \quad$ Agora temos $\left(a^{\tilde{\varphi}}\right)^{2}=\left(a^{\varphi} d^{\alpha_{1}}\right)^{2}=\left(a^{\varphi}\right)^{2}\left(d^{\alpha_{1}}\right)^{2}\left[a^{\varphi}, d\right]=1$. Analogamente, temos $\left(b^{\tilde{\varphi}}\right)^{2}=1$ e $\left(c^{\tilde{\varphi}}\right)^{2}=-1$. Então, para qualquer tripla $\left(\alpha_{1}, \alpha_{2}, \alpha_{3}\right), \operatorname{temos} \tilde{\varphi} \in \operatorname{Stab}\left(\lambda_{4}^{4}\right) . \operatorname{Logo}\left|\operatorname{Stab}\left(\lambda_{4}^{4}\right)\right|=8.8=64$. 
Consideremos os seguintes estabilizadores de $\lambda_{4}^{4}$ :

$$
\begin{gathered}
\sigma_{1}=\left(\begin{array}{llll}
0 & 1 & 0 & 0 \\
1 & 0 & 0 & 0 \\
0 & 1 & 1 & 0 \\
0 & 0 & 0 & 1
\end{array}\right) \quad \sigma_{2}=\left(\begin{array}{llll}
1 & 0 & 0 & 0 \\
0 & 1 & 0 & 0 \\
1 & 0 & 1 & 0 \\
0 & 0 & 0 & 1
\end{array}\right) \\
\rho_{1}=\left(\begin{array}{llll}
1 & 0 & 0 & 0 \\
0 & 1 & 0 & 1 \\
1 & 0 & 1 & 1 \\
0 & 0 & 0 & 1
\end{array}\right) \quad \rho_{2}=\left(\begin{array}{llll}
1 & 0 & 0 & 1 \\
0 & 1 & 0 & 0 \\
0 & 1 & 1 & 1 \\
0 & 0 & 0 & 1
\end{array}\right) \quad \rho_{3}=\left(\begin{array}{llll}
1 & 0 & 0 & 0 \\
0 & 1 & 0 & 0 \\
0 & 0 & 1 & 1 \\
0 & 0 & 0 & 1
\end{array}\right)
\end{gathered}
$$

Com cálculos diretos obtemos: $\sigma_{1}^{4}=1, \sigma_{2}^{2}=1, \sigma_{2} \sigma_{1} \sigma_{2}=\sigma_{1}^{3}, \rho_{1}^{2}=\rho_{2}^{2}=\rho_{3}^{2}=1$, $\rho_{i} \rho_{j}=\rho_{j} \rho_{i}, i, j=1,2,3$.

Sejam

$$
K_{1}=G R\left\{\sigma_{1}, \sigma_{2} \mid \sigma_{1}^{4}=1, \sigma_{2}^{2}=1, \sigma_{2} \sigma_{1} \sigma_{2}=\sigma_{1}^{3}\right\}
$$

e

$$
H_{1}=G R\left\{\rho_{1}, \rho_{2}, \rho_{3} \mid \rho_{1}^{2}=\rho_{2}^{2}=\rho_{3}^{2}=1, \rho_{i} \rho_{j}=\rho_{j} \rho_{i}, i, j=1,2,3\right\} .
$$

Os elementos destes dois grupos também são estabilizadores de $\lambda_{4}^{4}$. Na verdade, o produto de qualquer elemento de $K_{1}$ por $H_{1}$ é um estabilizador de $\lambda_{4}^{4}$. Logo temos $H_{1} \cap K_{1}=\{1\}$ e $\operatorname{Stab}\left(\lambda_{4}^{4}\right)=K_{1} H_{1}$.

Sabemos que $K_{1} \simeq D_{8}$ e $H_{1} \simeq \mathbb{Z}_{2}^{3}$. Temos ainda que $K_{1}$ não é um subgrupo normal de $\operatorname{Stab}\left(\lambda_{4}^{4}\right)$, pois por exemplo, para $\rho_{1} \in H_{1}$ e $\sigma_{1} \in K_{1}$ não temos $\rho_{1} \sigma_{1} \rho_{1} \in K_{1}$, ou seja, $\rho_{1} K_{1} \rho_{1} \nsubseteq K_{1}$. Por outro lado, $H_{1} \triangleleft \operatorname{Stab}\left(\lambda_{4}^{4}\right)$. Para provar isto, basta fazer os cálculos diretamente e usar as seguintes relações:

$\sigma_{2} \rho_{1}=\rho_{1} \sigma_{2}, \sigma_{2} \rho_{2}=\rho_{2} \sigma_{2}, \sigma_{1} \rho_{3}=\rho_{3} \sigma_{1}, \sigma_{2} \rho_{3}=\rho_{3} \sigma_{2}, \rho_{1} \sigma_{1}=\sigma_{1} \rho_{2}, \sigma_{1} \rho_{1} \rho_{3}=\rho_{2} \sigma_{1}$, $\rho_{1} \rho_{2} \sigma_{1}=\sigma_{1} \rho_{2} \rho_{1} \rho_{3}, \sigma_{1} \rho_{1}=\rho_{2} \rho_{3} \sigma_{1}, \sigma_{1} \rho_{2} \rho_{3}=\rho_{1} \rho_{3} \sigma_{1}, \sigma_{1} \rho_{1} \rho_{2}=\rho_{2} \rho_{3} \rho_{1} \sigma_{1}$. 
Portanto, $\operatorname{Stab}\left(\lambda_{4}^{4}\right) \simeq \mathbb{Z}_{2}^{3} \rtimes D_{8}$

5. Sejam $\lambda_{5}^{4}=(0000010100)$ e $\lambda_{5^{\prime}}^{3}=(000011)$. Neste caso, $\left|\operatorname{Stab}\left(\lambda_{5^{\prime}}^{3}\right)\right|=6$ pois $\lambda_{5^{\prime}}^{3} \in O_{5}^{3}$. As possibilidades para $\left(x=a^{\varphi}, y=b^{\varphi}, z=c^{\varphi}\right)$ são $(a, b, c),(a, a b, c)$, $(b, a, c),(b, a b, c),(a b, a, c),(a b, b, c)$. Claramente, $\left|\operatorname{Stab}\left(\lambda_{5}^{4}\right)\right|=6.8=48$.

Consideremos

$$
\sigma_{1}=\left(\begin{array}{llll}
1 & 0 & 0 & 0 \\
1 & 1 & 0 & 0 \\
0 & 0 & 1 & 0 \\
0 & 0 & 0 & 1
\end{array}\right) \quad \sigma_{2}=\left(\begin{array}{llll}
1 & 1 & 0 & 1 \\
0 & 1 & 0 & 0 \\
0 & 0 & 1 & 0 \\
0 & 0 & 0 & 1
\end{array}\right) \quad \sigma_{3}=\left(\begin{array}{llll}
1 & 0 & 0 & 0 \\
1 & 1 & 0 & 1 \\
0 & 0 & 1 & 0 \\
0 & 0 & 0 & 1
\end{array}\right) \quad \rho=\left(\begin{array}{llll}
1 & 0 & 0 & 0 \\
0 & 1 & 0 & 0 \\
0 & 0 & 1 & 1 \\
0 & 0 & 0 & 1
\end{array}\right)
$$

Temos que $\rho$ e $\sigma_{i}, i=1,2,3$, são estabilizadores de $\lambda_{5}^{4}$ de ordem 2. Ainda temos $\sigma_{1} \sigma_{3}=\sigma_{3} \sigma_{1}, \sigma_{1} \sigma_{2} \sigma_{1}=\sigma_{2} \sigma_{1} \sigma_{2}, \sigma_{2} \sigma_{3} \sigma_{2}=\sigma_{3} \sigma_{2} \sigma_{3}$ e $\rho \sigma=\sigma \rho$, para cada $\sigma \in S_{4}$. O grupo gerado por $\sigma_{i}, i=1,2,3$ é isomorfo a $S_{4}, G R\{\rho\} \simeq \mathbb{Z}_{2}$. Portanto, $\mathrm{OutC}_{5}^{4} \simeq S_{4} \times \mathbb{Z}_{2}$.

6. Sejam $\lambda_{6}^{4}=(1111110100)$ e $\lambda_{1}^{3}=(111111)$. Temos $\left|\operatorname{Stab}\left(\lambda_{1}^{3}\right)\right|=168$. Neste caso, somente para $\left(\alpha_{1}, \alpha_{2}, \alpha_{3}\right)=(0,0,0)$ teremos $\tilde{\varphi} \in \operatorname{Stab}\left(\lambda_{6}^{4}\right)$. De fato, $\left(a^{\varphi} d\right)^{2}=$ $\left(b^{\varphi} d\right)^{2}=\left(c^{\varphi} d\right)^{2}=1$, visto que $\left(a^{\varphi}\right)^{2}=\left(b^{\varphi}\right)^{2}=\left(c^{\varphi}\right)^{2}=-1$ e $d^{2}=-1$. Portanto, $\left|\operatorname{Stab}\left(\lambda_{6}^{4}\right)\right|=168$. Notemos que $a^{\varphi}, b^{\varphi}, c^{\varphi} \in\{a, b, c, a b, a c, b c, a b c\}$.

Consideremos os seguintes estabilizadores de $\lambda_{6}^{4}: \sigma_{1}, \sigma_{2}, \ldots, \sigma_{6}$, representados matricialmente por:

$$
\sigma_{1}=\left(\begin{array}{cccc}
1 & 1 & 0 & 0 \\
0 & 1 & 0 & 0 \\
0 & 0 & 1 & 0 \\
0 & 0 & 0 & 1
\end{array}\right) \quad \sigma_{2}=\left(\begin{array}{cccc}
1 & 0 & 1 & 0 \\
0 & 1 & 0 & 0 \\
0 & 0 & 1 & 0 \\
0 & 0 & 0 & 1
\end{array}\right) \quad \sigma_{3}=\left(\begin{array}{cccc}
1 & 0 & 0 & 0 \\
1 & 1 & 0 & 0 \\
0 & 0 & 1 & 0 \\
0 & 0 & 0 & 1
\end{array}\right)
$$




$$
\sigma_{4}=\left(\begin{array}{cccc}
1 & 0 & 0 & 0 \\
0 & 1 & 1 & 0 \\
0 & 0 & 1 & 0 \\
0 & 0 & 0 & 1
\end{array}\right) \quad \sigma_{5}=\left(\begin{array}{cccc}
1 & 0 & 0 & 0 \\
0 & 1 & 0 & 0 \\
1 & 0 & 1 & 0 \\
0 & 0 & 0 & 1
\end{array}\right) \quad \sigma_{6}=\left(\begin{array}{cccc}
1 & 0 & 0 & 0 \\
0 & 1 & 0 & 0 \\
0 & 1 & 1 & 0 \\
0 & 0 & 0 & 1
\end{array}\right)
$$

Temos $a^{\sigma_{1}}=a b, b^{\sigma_{1}}=b, c^{\sigma_{1}}=c$ e $d^{\sigma_{1}}=d$. Logo $\sigma_{1} \in \operatorname{Stab}\left(\lambda_{6}^{4}\right)$. Analogamente, vemos que $\sigma_{2}, \ldots, \sigma_{6} \in \operatorname{Stab}\left(\lambda_{6}^{4}\right)$. Qualquer produto dos $\sigma_{i}^{\prime} \mathrm{s}, i=1, \ldots, 6$ é um estabilizador de $\lambda_{6}^{4}$. Logo $G R\left\{\sigma_{1}, \ldots, \sigma_{6}\right\}=\operatorname{Stab}\left(\lambda_{6}^{4}\right), \operatorname{mas} G R\left\{\sigma_{1}, \ldots, \sigma_{6}\right\} \simeq$ $G L_{3}(2)$, portanto, $\operatorname{Stab}\left(\lambda_{6}^{4}\right) \simeq G L_{3}(2)$.

7. Neste caso, temos $\lambda_{7}^{4}=(0001000000), \lambda_{2}^{3}=(000000)$ e $\left|\operatorname{Stab}\left(\lambda_{2}^{3}\right)\right|=24$. Analogamente ao caso anterior só teremos $\tilde{\varphi} \in \operatorname{Stab}\left(\lambda_{7}^{4}\right)$ para $\left(\alpha_{1}, \alpha_{2}, \alpha_{3}\right)=(0,0,0)$. Portanto, $\left|\operatorname{Stab}\left(\lambda_{7}^{4}\right)\right|=24$.

Sejam $\sigma_{1}=\left(\begin{array}{cccc}1 & 0 & 0 & 0 \\ 0 & 0 & 1 & 0 \\ 0 & 1 & 0 & 0 \\ 0 & 0 & 0 & 1\end{array}\right), \sigma_{2}=\left(\begin{array}{cccc}0 & 1 & 0 & 0 \\ 1 & 0 & 0 & 0 \\ 0 & 0 & 1 & 0 \\ 0 & 0 & 0 & 1\end{array}\right), \sigma_{3}=\left(\begin{array}{cccc}1 & 0 & 0 & 0 \\ 1 & 1 & 0 & 0 \\ 1 & 0 & 1 & 0 \\ 0 & 0 & 0 & 1\end{array}\right)$. Temos:

$$
\begin{array}{r}
\sigma_{1}^{2}=\sigma_{2}^{2}=\sigma_{3}^{2}=1, \\
\sigma_{1} \sigma_{2} \sigma_{1}=\sigma_{2} \sigma_{1} \sigma_{2}, \\
\sigma_{3} \sigma_{2} \sigma_{3}=\sigma_{2} \sigma_{3} \sigma_{2}, \\
\sigma_{1} \sigma_{3}=\sigma_{3} \sigma_{1} .
\end{array}
$$

$\operatorname{Como} \sigma_{1}, \sigma_{2}, \sigma_{3} \in \operatorname{Stab}\left(\lambda_{7}^{4}\right)$, então $\operatorname{Stab}\left(\lambda_{7}^{4}\right)=G R\left\{\sigma_{1}, \sigma_{2}, \sigma_{3} \mid \sigma_{1}^{2}=\sigma_{2}^{2}=\sigma_{3}^{2}=\right.$ $\left.1, \sigma_{1} \sigma_{2} \sigma_{1}=\sigma_{2} \sigma_{1} \sigma_{2}, \sigma_{3} \sigma_{2} \sigma_{3}=\sigma_{2} \sigma_{3} \sigma_{2}, \sigma_{1} \sigma_{3}=\sigma_{3} \sigma_{1}\right\}$, mas o grupo gerado por $\sigma_{1}, \sigma_{2}, \sigma_{3}$ e que satisfaz as relações (2.1)-(2.4) é isomorfo ao grupo das permutações de 4 elementos, $S_{4}$. Portanto, $\operatorname{Stab}\left(\lambda_{7}^{4}\right) \simeq S_{4}$. 
8. Sejam $\lambda_{8}^{4}=(0000001000)$ e $\lambda_{2}^{3}=(000000)$. Suponhamos $\sigma \in \operatorname{Stab}\left(\lambda_{8}^{4}\right)$ e $a^{\sigma}=u, b^{\sigma}=v, c^{\sigma}=w, d^{\sigma}=d$. Então

$$
\begin{array}{r}
u^{2}=v^{2}=w^{2}=1 ; d^{2}=1 \\
{[u, v]=[u, w]=1} \\
{[v, w]=[v, d]=[w, d]=1} \\
{[u, d]=-1}
\end{array}
$$

Por (2.5) e (2.8) temos $u \in\{a, a b, a c, a b c d\}$ e por (2.5) e (2.7) temos $v, w \in\{b, c, b c, b d, c d, b c d\}$. Logo por (2.6) obtemos todas as possibilidades possíveis para $(u, v, w)$. Por exemplo, para $u=a$ temos $(u, v, w)=(a, b, c)$, $(a, b, b c d),(a, c, b),(a, c, b c d),(a, b c d, b)$ ou $(a, b c d, c)$. Para qualquer $u$ obtemos 6 bases diferentes. Portanto, no total temos 24 bases diferentes e assim, $\left|\operatorname{Stab}\left(\lambda_{8}^{4}\right)\right|=24$.

\section{Observação:}

Sabemos que $\left|\operatorname{Stab}\left(\lambda_{2}^{3}\right)\right|=24$. Suponhamos $u=a^{\varphi} d^{\alpha_{1}}, v=b^{\varphi} d^{\alpha_{2}}, w=c^{\varphi} d^{\alpha_{3}}$, $\alpha_{i} \in\{0,1\}, i=1,2,3$. Analisando o caso em que $\left(\alpha_{1}, \alpha_{2}, \alpha_{3}\right)=(0,0,0)$, é a bc

importante observar que, por exemplo, $\varphi$ definida por $b \stackrel{\varphi}{\longmapsto} a c$ é tal que

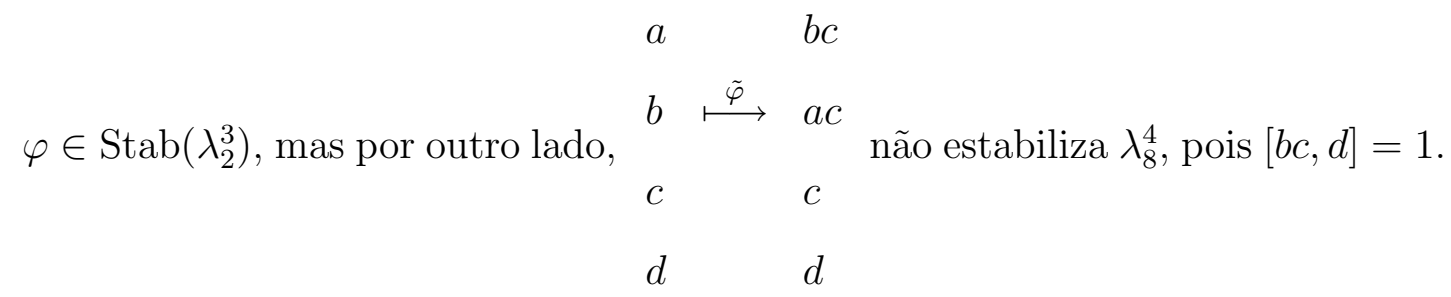

Pelo que vimos acima, temos apenas 6 estabilizadores de $\lambda_{8}^{4} \operatorname{sendo} \varphi \in \operatorname{Stab}\left(\lambda_{8}^{3}\right)$, dados por: $(u, v, w, d)=(a b, b c, b, d),(a b, b, b c, d),(a c, b c, c, d),(a c, c, b c, d),(a, b . c, d)$ e $(a, c, b, d)$. 
Além disso, se supormos, por exemplo, $\left(\alpha_{1}, \alpha_{2}, \alpha_{3}\right)=(1,1,0)$ teremos, por um lado, $\left[a^{\varphi} d, b^{\varphi} d\right]=\left[a^{\varphi}, d\right]\left[d, b^{\varphi}\right]=1$, ou seja, ou $\left[a^{\varphi}, d\right]=\left[d, b^{\varphi}\right]=1$ ou $\left[a^{\varphi}, d\right]=$ $\left[d, b^{\varphi}\right]=-1$. Por outro lado, $\left[a^{\varphi}, d\right]=-1$ e $\left[d, b^{\varphi}\right]=1$ para termos $\left[a^{\varphi} d, d\right]=-1$ $\mathrm{e}\left[b^{\varphi} d, d\right]=1$. Analogamente, para qualquer outra possibilidade de $\left(\alpha_{1}, \alpha_{2}, \alpha_{3}\right) \neq$ $(0,0,0)$, não teremos mais estabilizadores de $\lambda_{8}^{4}$ partindo da hipótese de que $\varphi \in \operatorname{Stab}\left(\lambda_{2}^{3}\right)$.

Para mostrar que $\operatorname{Out}\left(C_{8}^{4}\right) \simeq S_{4}$, basta considerar os estabilizadores:

$$
\sigma_{1}=\left(\begin{array}{cccc}
1 & 0 & 0 & 0 \\
0 & 1 & 0 & 0 \\
0 & 1 & 1 & 1 \\
0 & 0 & 0 & 1
\end{array}\right), \sigma_{2}=\left(\begin{array}{cccc}
1 & 0 & 0 & 0 \\
0 & 1 & 1 & 1 \\
0 & 0 & 1 & 0 \\
0 & 0 & 0 & 1
\end{array}\right), \sigma_{3}=\left(\begin{array}{cccc}
1 & 1 & 0 & 0 \\
0 & 1 & 0 & 0 \\
0 & 1 & 1 & 0 \\
0 & 0 & 0 & 1
\end{array}\right) .
$$

9. Sejam $\lambda_{9}^{4}=(0100001000)$ e $\sigma \in \operatorname{Stab}\left(\lambda_{9}^{4}\right)$ definido como acima. Neste caso:

$$
\begin{array}{r}
u^{2}=w^{2}=d^{2}=1 ; v^{2}=-1 \\
{[u, v]=[u, w]=1} \\
{[v, w]=[v, d]=[w, d]=1} \\
{[u, d]=-1}
\end{array}
$$

Por (2.9) e (2.12), temos $u \in\{a, a c, a b c, a b d\}$ e por (2.9) e (2.11) temos $v \in\{b, b c, b d, b c d\}$ e $w \in\{c, c d\}$. Agora por (2.10) temos que $(u, v, w)$ são dados por $(a, b, c),(a, b c d, c),(a c, b c, c),(a c, b d, c),(a b c, b d, c d),(a b c, b c d, c d),(a b d, b, c d)$, $(a b d, b c, c d)$. Portanto, $\left|\operatorname{Stab}\left(\lambda_{9}^{4}\right)\right|=8$.

Sejam $\sigma=\left(\begin{array}{cccc}1 & 0 & 0 & 0 \\ 0 & 1 & 1 & 1 \\ 0 & 0 & 1 & 0 \\ 0 & 0 & 0 & 1\end{array}\right)$ e $\rho=\left(\begin{array}{cccc}1 & 1 & 1 & 0 \\ 0 & 1 & 1 & 1 \\ 0 & 0 & 1 & 1 \\ 0 & 0 & 0 & 1\end{array}\right)$. Com cálculos diretos obtemos que as ordens de $\sigma$ e $\rho$ são 2 e 4, respectivamente, e vale a seguinte relação $\sigma \rho \sigma^{-1}=\rho^{-1}$, ou melhor, $\rho \sigma=\sigma \rho^{3}$. Temos que $\sigma$ e $\rho$ estabilizam $\lambda_{9}^{4}$. Logo 
$G R\left\{\sigma, \rho \mid \rho^{4}=1, \sigma^{2}=1, \sigma \rho \sigma^{-1}=\rho^{-1}\right\}=\operatorname{Stab}\left(\lambda_{9}^{4}\right)$. Portanto, $\operatorname{Stab}\left(\lambda_{9}^{4}\right) \simeq D_{8}$.

10. Sejam $\lambda_{10}^{4}=(0001111000)$ e $\lambda_{5^{\prime \prime}}^{3}=(000110)$. Temos $\left|\operatorname{Stab}\left(\lambda_{5^{\prime \prime}}^{3}\right)\right|=6$. De fato, se $\varphi \in \operatorname{Stab}\left(\lambda_{5^{\prime \prime}}^{3}\right)$, então as possibilidades para $\lambda_{5^{\prime \prime}}^{3}$ são $\left(a^{\varphi}, b^{\varphi}, c^{\varphi}\right)=(a, b c, c)$, $(a, c, b c),(a, b, b c),(a, b c, b),(a, c, b)$ ou $(a, b, c)$.

Seja $\tilde{\varphi}$ como antes e suponhamos $\left(\alpha_{1}, \alpha_{2}, \alpha_{3}\right)=(0,0,0)$. Neste caso, temos 6 estabilizadores de $\lambda_{10}^{4}$ diferentes dos dados por $\left(a^{\varphi}, b^{\varphi}, c^{\varphi}, d\right)$, visto que $a^{\varphi}=a$, $b^{\varphi}=b, c$ ou $b c$ e $c^{\varphi}=b, c$ ou $b c$. Agora supondo $\left(\alpha_{1}, \alpha_{2}, \alpha_{3}\right)=(1,0,0)$, temos que $\tilde{\varphi}$ é tal que $\left(a^{\tilde{\varphi}}, b^{\tilde{\varphi}}, c^{\tilde{\varphi}}, d^{\tilde{\varphi}}\right)=\left(a^{\varphi} d, b^{\varphi}, c^{\varphi}, d\right)$ e assim, $\left(a^{\varphi} d\right)^{2}=\left(b^{\varphi}\right)^{2}=\left(c^{\varphi}\right)^{2}=1$, $\left[a^{\varphi} d, b^{\varphi}\right]=\left[a^{\varphi} d, c^{\varphi}\right]=\left[a^{\varphi} d, d\right]=-1 \mathrm{e}\left[b^{\varphi}, d\right]=\left[c^{\varphi}, d\right]=\left[b^{\varphi}, c^{\varphi}\right]=1$. Logo, temos mais 6 estabilizadores de $\lambda_{10}^{4}$.

Para qualquer outra tripla $\left(\alpha_{1}, \alpha_{2}, \alpha_{3}\right)$ não teremos mais estabilizadores de $\lambda_{10}^{4}$, pois se, por exemplo, $\left(\alpha_{1}, \alpha_{2}, \alpha_{3}\right)=(0,1,0)$ teremos $b^{\tilde{\varphi}}=b^{\varphi} d$, $\operatorname{mas}\left(b^{\varphi} d\right)^{2}=-1$ visto que $d^{2}=-1$ e $\left(b^{\varphi}\right)^{2}=\left[b^{\varphi}, d\right]=1$. A análise é análoga para as outras possibilidades de $\left(\alpha_{1}, \alpha_{2}, \alpha_{3}\right)$.

Portanto, $\left|\operatorname{Stab}\left(\lambda_{10}^{4}\right)\right|=12$.

Considere os seguintes estabilizadores de $\lambda_{10}^{4}$ :

$$
\sigma_{1}=\left(\begin{array}{cccc}
1 & 0 & 0 & 0 \\
0 & 1 & 0 & 0 \\
0 & 1 & 1 & 0 \\
0 & 0 & 0 & 1
\end{array}\right) \quad \sigma_{2}=\left(\begin{array}{cccc}
1 & 0 & 0 & 0 \\
0 & 1 & 1 & 0 \\
0 & 0 & 1 & 0 \\
0 & 0 & 0 & 1
\end{array}\right) \quad \rho=\left(\begin{array}{cccc}
1 & 0 & 0 & 1 \\
0 & 1 & 0 & 0 \\
0 & 0 & 1 & 0 \\
0 & 0 & 0 & 1
\end{array}\right)
$$

Temos $\sigma_{1}^{2}=\sigma_{2}^{2}=\rho^{2}=1$ e $\sigma \rho=\rho \sigma$, para cada $\sigma \in S_{3} \cdot \mathbb{Z}_{2}$.

Portanto, Out $C_{10}^{4} \simeq S_{3} \times \mathbb{Z}_{2}$.

11. Seja $\lambda_{11}^{4}=(0001001000)$. Temos $u^{2}=v^{2}=w^{2}=1, d^{2}=-1,[u, v]=$ $[u, w]=1,[v, w]=[v, d]=[w, d]=1 \mathrm{e}[u, d]=-1$. Deste modo, obtemos $u \in\{a, a b, a c, a d, a b d, a c d\}$ e $v, w \in\{b, c, b c\} . \operatorname{Assim},\left|\operatorname{Stab}\left(\lambda_{11}^{4}\right)\right|=12$. 
Consideremos os seguintes estabilizadores de $\lambda_{11}^{4}$ :

$\sigma=\left(\begin{array}{llll}1 & 0 & 0 & 0 \\ 0 & 0 & 1 & 0 \\ 0 & 1 & 0 & 0 \\ 0 & 0 & 0 & 1\end{array}\right), \theta=\left(\begin{array}{llll}1 & 1 & 0 & 0 \\ 0 & 1 & 0 & 0 \\ 0 & 1 & 1 & 0 \\ 0 & 0 & 0 & 1\end{array}\right)$ e $\rho=\left(\begin{array}{cccc}1 & 0 & 0 & 0 \\ 0 & 1 & 0 & 0 \\ 0 & 0 & 1 & 0 \\ 0 & 0 & 0 & 1\end{array}\right)$.

Com simples cálculos obtemos $\sigma^{2}=\theta^{2}=\rho^{2}=1, \sigma \theta \sigma=\theta \sigma \theta, \sigma \theta \neq \theta \sigma$, $\sigma \rho=\rho \sigma, \theta \rho=\rho \theta, \sigma \theta \rho=\rho \sigma \theta, \sigma \theta \sigma \rho=\rho \sigma \theta \sigma$ e $\theta \sigma \rho=\rho \theta \sigma$. Todos estes elementos são estabilizadores de $\lambda_{11}^{4}$. Sejam $H=G R\left\{\sigma, \theta \mid \sigma^{2}=\theta^{2}, \sigma \theta \sigma=\theta \sigma \theta\right\}$ e $K=G R\left\{\rho \mid \rho^{2}=1\right\}$. Logo, pelos cálculos anteriores, qualquer estabilizador de $\lambda_{11}^{4}$ é escrito de maneira única como o produto de um elemento de $H$ por um elementode $K$ e $x y=y x$, para todo $x \in H, y \in K$. Além disso, sabemos que $H \cong S_{3}$ e $K \cong \mathbb{Z}_{2}$. Portanto, pelo Lema 2.3.1 temos $\operatorname{Stab}\left(\lambda_{11}^{4}\right) \simeq S_{3} \times \mathbb{Z}_{2}$.

12. Seja $\lambda_{12}^{4}=(0000001100)$. Temos $u^{2}=v^{2}=w^{2}=d^{2}=1,[u, v]=[u, w]=[v, d]=$ $[w, d]=1$ e $[u, d]=[v, w]=-1$. Deste modo, obtemos $u \in\{a, a b, a c, a b c\}$ e $v, w \in\{b, c\}$. Assim, $\left|\operatorname{Stab}\left(\lambda_{12}^{4}\right)\right|=8$.

Neste caso, consideremos $\sigma=\left(\begin{array}{cccc}1 & 0 & 0 & 0 \\ 0 & 0 & 1 & 0 \\ 0 & 1 & 0 & 0 \\ 0 & 0 & 0 & 1\end{array}\right)$ e $\rho=\left(\begin{array}{cccc}1 & 1 & 0 & 0 \\ 0 & 0 & 1 & 0 \\ 0 & 1 & 0 & 0 \\ 0 & 0 & 0 & 1\end{array}\right)$,

estabilizadores de $\operatorname{Stab}\left(\lambda_{12}^{4}\right)$. Analogamente a análise do caso 9, temos $G R\left\{\sigma, \rho \mid \rho^{4}=1, \sigma^{2}=1, \sigma \rho \sigma^{-1}=\rho^{-1}\right\}=\operatorname{Stab}\left(\lambda_{12}^{4}\right)$.

Portanto, $\operatorname{Stab}\left(\lambda_{12}^{4}\right) \simeq D_{8}$.

13. Seja $\lambda_{13}^{4}=(0110111100)$. Neste caso, $u^{2}=d^{2}=1, v^{2}=w^{2}=-1,[u, v]=$ $[u, w]=[u, d]=[v, w]=-1,[v, d]=[w, d]=1$. Daí temos $u \in\{a, a b, a c, a b c\} \mathrm{e}$ $v, w \in\{b, c, b c\}$. Logo, $\left|\operatorname{Stab}\left(\lambda_{13}^{4}\right)\right|=24$. 
Aqui os geradores necessários são dados por:

$$
\sigma_{1}=\left(\begin{array}{cccc}
1 & 0 & 0 & 0 \\
0 & 1 & 0 & 0 \\
0 & 1 & 1 & 0 \\
0 & 0 & 0 & 1
\end{array}\right) \quad \sigma_{2}=\left(\begin{array}{cccc}
1 & 0 & 0 & 0 \\
0 & 0 & 1 & 0 \\
0 & 1 & 0 & 0 \\
0 & 0 & 0 & 1
\end{array}\right) \quad \sigma_{3}=\left(\begin{array}{cccc}
1 & 1 & 0 & 0 \\
0 & 1 & 0 & 0 \\
0 & 1 & 1 & 0 \\
0 & 0 & 0 & 1
\end{array}\right)
$$

Analogamente a casos anteriores se mostra que $\operatorname{Out}\left(C_{13}^{4}\right) \simeq S_{4}$.

14. Para $\lambda_{14}^{4}=(0001001100)$ temos $u^{2}=v^{2}=w^{2}=1, d^{2}=-1,[u, v]=[u, w]=1$, $[v, d]=[w, d]=1$ e $[u, d]=[v, w]=-1 \mathrm{e}$, assim,

$$
u \in\{a, a b, a c, a d, a b c, a b d, a c d, a b c d\} \text { e } v, w \in\{b, c, b c d\} .
$$

Portanto, $\left|\operatorname{Stab}\left(\lambda_{14}^{4}\right)\right|=48$.

Considere os seguintes geradores para $\mathbb{Z}_{2}^{3}$ :

$$
\rho_{1}=\left(\begin{array}{cccc}
1 & 1 & 0 & 0 \\
0 & 1 & 0 & 0 \\
0 & 0 & 1 & 0 \\
0 & 0 & 0 & 1
\end{array}\right) \quad \rho_{2}=\left(\begin{array}{cccc}
1 & 0 & 0 & 1 \\
0 & 1 & 0 & 0 \\
0 & 0 & 1 & 0 \\
0 & 0 & 0 & 1
\end{array}\right) \quad \rho_{3}=\left(\begin{array}{cccc}
1 & 0 & 1 & 0 \\
0 & 1 & 0 & 0 \\
0 & 0 & 1 & 0 \\
0 & 0 & 0 & 1
\end{array}\right)
$$

Para $S_{3}$, considere: $\sigma_{1}=\left(\begin{array}{cccc}1 & 0 & 0 & 0 \\ 0 & 0 & 1 & 0 \\ 0 & 1 & 0 & 0 \\ 0 & 0 & 0 & 1\end{array}\right) \quad \sigma_{2}=\left(\begin{array}{cccc}1 & 1 & 0 & 1 \\ 0 & 1 & 0 & 0 \\ 0 & 1 & 1 & 1 \\ 0 & 0 & 0 & 1\end{array}\right)$

Fazendo todos os cálculos necessários obtemos Out $C_{14}^{4} \simeq \mathbb{Z}_{2}^{3} \rtimes S_{3}$.

15. Para $\lambda_{15}^{4}=(1001001100)$ temos $u^{2}=-1, v^{2}=w^{2}=1, d^{2}=-1,[u, v]=[u, w]=$ $[v, d]=[w, d]=1$ e $[u, d]=[v, w]=-1$, e assim,

$u \in\{a, a b, a c, a d, a b c, a b d, a c d, a b c d\}$ e $v, w \in\{b, c, b c d\}$. 
Portanto, $\left|\operatorname{Stab}\left(\lambda_{15}^{4}\right)\right|=48$.

Considere os seguintes geradores para $\mathbb{Z}_{2}^{3}$ :

$$
\rho_{1}=\left(\begin{array}{cccc}
1 & 1 & 0 & 0 \\
0 & 1 & 0 & 0 \\
0 & 0 & 1 & 0 \\
0 & 0 & 0 & 1
\end{array}\right) \quad \rho_{2}=\left(\begin{array}{cccc}
1 & 0 & 0 & 1 \\
0 & 1 & 0 & 0 \\
0 & 0 & 1 & 0 \\
0 & 0 & 0 & 1
\end{array}\right) \quad \rho_{3}=\left(\begin{array}{cccc}
1 & 0 & 1 & 0 \\
0 & 1 & 0 & 0 \\
0 & 0 & 1 & 0 \\
0 & 0 & 0 & 1
\end{array}\right)
$$

Para $S_{3}$, considere: $\sigma_{1}=\left(\begin{array}{cccc}1 & 0 & 0 & 0 \\ 0 & 0 & 1 & 0 \\ 0 & 1 & 0 & 0 \\ 0 & 0 & 0 & 1\end{array}\right) \quad \sigma_{2}=\left(\begin{array}{cccc}1 & 1 & 0 & 1 \\ 0 & 1 & 0 & 0 \\ 0 & 1 & 1 & 1 \\ 0 & 0 & 0 & 1\end{array}\right)$

Neste caso, também obtemos Out $C_{15}^{4} \simeq \mathbb{Z}_{2}^{3} \rtimes S_{3}$.

16. Seja $\lambda_{16}^{4}=(0001111100)$, então $u^{2}=v^{2}=w^{2}=1, d^{2}=-1,[u, v]=[u, w]=-1$, $[u, d]=[v, w]=-1$ e $[v, d]=[w, d]=1$, e assim, $u \in\{a, a b c, a d, a b c d\}$ e $v, w \in\{b, c\}$. Portanto, $\left|\operatorname{Stab}\left(\lambda_{16}^{4}\right)\right|=8$.

Sejam os seguintes estabilizadores de $\lambda_{16}^{4}$ :

$$
\sigma=\left(\begin{array}{llll}
1 & 0 & 0 & 0 \\
0 & 0 & 1 & 0 \\
0 & 1 & 0 & 0 \\
0 & 0 & 0 & 1
\end{array}\right), \theta=\left(\begin{array}{llll}
1 & 0 & 0 & 1 \\
0 & 1 & 0 & 0 \\
0 & 0 & 1 & 0 \\
0 & 0 & 0 & 1
\end{array}\right) \text { e } \rho=\left(\begin{array}{llll}
1 & 1 & 1 & 0 \\
0 & 0 & 1 & 0 \\
0 & 1 & 0 & 0 \\
0 & 0 & 0 & 1
\end{array}\right) .
$$

Temos $\sigma^{2}=\theta^{2}=\rho^{2}=1$ e $\sigma \theta=\theta \sigma, \sigma \rho=\rho \sigma$ e $\sigma \rho=\rho \sigma$. Claramente, $\operatorname{Stab}\left(\lambda_{16}^{4}\right) \simeq \mathbb{Z}_{2}^{3}$ 


\section{Capítulo 3}

\section{Representações dos Loops de Código}

No primeiro capítulo, definimos loops de código a partir de códigos pares fixados. Neste capítulo, fixado um loop de código $L$, pretendemos determinar códigos pares $V$ tais que $L \simeq L(V)$.

Definição 3.0.1. Uma representação de um loop de código $L$ é um código par $V \subseteq \mathbf{F}_{2}^{m}$ tal que $L \simeq L(V)$. O grau da representação é o número gr $V=m$.

Observemos que existem várias representações diferentes para um mesmo loop de código. Mostraremos pelos Teoremas 3.2.1 e 3.3.1 que, existem representações dos loops de código não associativos de posto 3 e 4 tais que o grau de cada uma destas representações seja o menor possível, ou seja, minimal.

Definição 3.0.2. Uma representação $V$ é chamada básica se gr $V$ é minimal.

Se identificarmos o $\mathbf{F}_{2}$-espaço $\mathbf{F}_{2}^{m}$ com o conjunto de todos os subconjuntos de $I_{m}=\{1, \ldots, m\}$ então teremos uma equivalência $\sim$ sobre $I_{m}$ tal que $i \sim j$ se, e somente se, $\{i, j\} \cap v=\{i, j\}$ ou $\{i, j\} \cap v=\emptyset$, para todo $v \in V$. Notemos que esta 
definição é equivalente a seguinte: $i \sim j$ se, e somente se, $\{i, j\} \cap v_{k}=\{i, j\}$ ou $\{i, j\} \cap v_{k}=\emptyset, k=1, \ldots, s$ e $\left\{v_{1}, \ldots, v_{s}\right\}$ é uma base de $V$.

Consideraremos somente representações tais que, para qualquer classe de equivalência $X$, temos $|X|<8$. Chamaremos estas representações de reduzidas.

Nosso principal problema é encontrar todas as representações básicas para um dado loop de código.

Definição 3.0.3. Para uma dada representação $V$ e todas as classes de equivalência $X_{1}, \ldots, X_{r}$, o tipo de $V$ é um vetor $\left(\left|X_{1}\right|, \ldots,\left|X_{r}\right|\right)$ tal que $\left|X_{1}\right| \leq\left|X_{2}\right| \leq \ldots \leq\left|X_{r}\right|$.

Definição 3.0.4. Sejam $V_{1}$ e $V_{2}$ códigos pares de $\mathbf{F}_{2}^{m}$. Dizemos que $V_{1}$ e $V_{2}$ são códigos pares isomorfos se, e somente se, existe uma bijeção $\varphi \in S_{m}$ (grupo de permutações de $m$ elementos) tal que $V_{1}^{\varphi}=V_{2}$.

\subsection{Representações reduzidas dos Loops de Código de posto 3}

De acordo com o Teorema 2.1.1, temos exatamente 5 loops de código não associativos de posto 3 , a saber, $C_{1}^{3}, C_{2}^{3}, C_{3}^{3}, C_{4}^{3}, C_{5}^{3}$. Para cada $C_{i}^{3}, i=1, \ldots, 5$, encontraremos todas as representações reduzidas, e as denotaremos por $V_{i}^{3}$.

Seja $X=\{a, b, c\}$ um conjunto de geradores de $C_{i}^{3}$ tal que $\lambda_{X}\left(C_{i}^{3}\right)=\lambda\left(C_{i}^{3}\right)$ seja seu vetor característico correspondente. Assim,

$$
\begin{aligned}
& \lambda\left(C_{1}^{3}\right)=(1,1,1,1,1,1) \\
& \lambda\left(C_{2}^{3}\right)=(0,0,0,0,0,0) \\
& \lambda\left(C_{3}^{3}\right)=(0,0,0,1,1,1) \\
& \lambda\left(C_{4}^{3}\right)=(1,1,0,0,0,0) \\
& \lambda\left(C_{5}^{3}\right)=(1,0,0,0,0,0) .
\end{aligned}
$$


Seja $\left\{v_{1}, v_{2}, v_{3}\right\}$ um conjunto de geradores da representação reduzida $V_{i}^{3}$, onde $v_{1}, v_{2}, v_{3}$ correspondem a $a, b, c$ respectivamente.

Adotaremos a notação: $t_{123}=\left|v_{1} \cap v_{2} \cap v_{3}\right|$.

Observemos que:

1. Para $\left|v_{i}\right| \equiv 0(\bmod 4)$ :

(a) Caso $v_{i}^{2}=-1$ temos $\left|v_{i}\right| \equiv 4(\bmod 8)$;

(b) Caso $v_{i}^{2}=1$ temos $\left|v_{i}\right| \equiv 0(\bmod 8)$.

De fato, por definição, $\left|v_{i}\right|=4 k$, para algum $k$ inteiro não-negativo e pelo Teorema 1.2.1 temos $v_{i}^{2}=(-1)^{\frac{\left|v_{i}\right|}{4}}, \operatorname{logo} v_{i}^{2}=(-1)^{k}$. Se $v_{i}^{2}=-1$, então existe $j$ tal que $k=2 j+1$ e $\operatorname{assim}\left|v_{i}\right| \equiv 4(\bmod 8)$. Se $v_{i}^{2}=1$, então existe $j$ tal que $k=2 j$ e assim $\left|v_{i}\right| \equiv 0(\bmod 8)$.

2. Para $\left|v_{i} \cap v_{j}\right| \equiv 0(\bmod 2)$ :

(a) Caso $\left[v_{i}, v_{j}\right]=-1$ temos $\left|v_{i} \cap v_{j}\right| \equiv 2(\bmod 4)$;

(b) Caso $\left[v_{i}, v_{j}\right]=1$ temos $\left|v_{i} \cap v_{j}\right| \equiv 0(\bmod 4)$.

De fato, por definição, $\left|v_{i} \cap v_{j}\right|=2 l$, para algum $l$ inteiro não-negativo, e pelo Teorema 1.2.1 $\left[v_{i}, v_{j}\right]=(-1)^{\frac{\left|v_{i} \cap v_{j}\right|}{2}}, \operatorname{logo}\left[v_{i}, v_{j}\right]=(-1)^{l}$. Se $\left[v_{i}, v_{j}\right]=-1$, então $l=2 s+1$, para algum $s$ inteiro não-negativo, de onde $\left|v_{i} \cap v_{j}\right| \equiv$ $2(\bmod 4)$. Analogamente, se $\left[v_{i}, v_{j}\right]=1$, teremos $\left|v_{i} \cap v_{j}\right| \equiv 0(\bmod 4)$.

3. Como $\left(v_{1}, v_{2}, v_{3}\right)=-1$, então temos $t_{123} \equiv 1(\bmod 2)$.

De fato, pela definição de $\left(v_{1}, v_{2}, v_{3}\right)$ temos $(-1)^{t_{123}}=-1$, logo existe um inteiro positivo $k$ tal que $t_{123}=2 k+1$, ou seja, $t_{123} \equiv 1(\bmod 2)$.

Neste caso não acontece $v_{i} \subset v_{j}, v_{j} \subset v_{i}, i \neq j$ ou $v_{i} \cap v_{j}=\emptyset$. Se $v_{1} \subset v_{2}$, por exemplo, teríamos $t_{123} \equiv\left|v_{1} \cap v_{3}\right| \equiv 0(\bmod 2)$, o que seria um absurdo pelo que provamos acima. Se $v_{2} \cap v_{3}=\emptyset$, por exemplo, teríamos $t_{123} \equiv\left|v_{1}\right| \equiv 0(\bmod 4)$, que também não pode acontecer. 


\section{Técnica para encontrar uma representação reduzida qualquer}

Suponhamos que o grau de $V_{i}^{3}$ seja $m$ e consideremos $X_{i}$ uma classe de equivalência sobre $I_{m}=\{1,2, \ldots, m\}$ tal que $\left|X_{i}\right|<8$. Os elementos de $V_{i}^{3}$ são considerados como subconjuntos de $I_{m}$. Como $i \sim j$ se, e somente se, $\{i, j\} \cap v_{k}=\{i, j\}$ ou $\{i, j\} \cap v_{k}=\emptyset$, para $k=1,2,3$, e $\left\{v_{1}, v_{2}, v_{3}\right\}$ é uma base de $V_{i}^{3}$, temos então apenas 7 classes de equivalência dadas pelos elementos dos seguintes conjuntos $v_{1} \cap$ $v_{2} \cap v_{3},\left(v_{1} \cap v_{2}\right) \backslash\left(v_{1} \cap v_{2} \cap v_{3}\right),\left(v_{1} \cap v_{3}\right) \backslash\left(v_{1} \cap v_{2} \cap v_{3}\right),\left(v_{2} \cap v_{3}\right) \backslash\left(v_{1} \cap v_{2} \cap v_{3}\right)$, $v_{1} \backslash\left(\left(v_{1} \cap v_{2}\right) \cup\left(v_{1} \cap v_{3}\right)\right), v_{2} \backslash\left(\left(v_{1} \cap v_{2}\right) \cup\left(v_{2} \cap v_{3}\right)\right)$ e $v_{3} \backslash\left(\left(v_{1} \cap v_{3}\right) \cup\left(v_{2} \cap v_{3}\right)\right)$, denotadas respectivamente por $X_{1}, \ldots, X_{7}$. Como cada classe tem no máximo 7 elementos, então $t_{123}=1,3,5$ ou $7,\left|v_{i} \cap v_{j}\right|=2,4,6,10$ ou 14 e $\left|v_{i}\right|=4,8,12,16,20,24$ ou 28. Logo, $7 \leq m \leq 49$.

Vamos apresentar uma técnica para encontrar representações reduzidas de posto 3, encontrando os valores de $\left|v_{1}\right|,\left|v_{2}\right|,\left|v_{3}\right|,\left|v_{1} \cap v_{2}\right|,\left|v_{1} \cap v_{3}\right|,\left|v_{2} \cap v_{3}\right|$ e $t_{123}$ :

1. Fixe $\left|v_{1}\right|$ e determine as possibilidades para $\left|v_{1} \cap v_{2}\right|$ e $\left|v_{1} \cap v_{3}\right|$;

2. Escolha $\left|v_{1} \cap v_{2}\right|$ e $\left|v_{1} \cap v_{3}\right|$ e determine os valores possíveis para $t_{123}$ de modo que $\left|v_{1} \cap v_{2}\right|-t_{123}<8$ e $\left|v_{1} \cap v_{3}\right|-t_{123}<8 ;$

3. Verifique se $\left|v_{1} \cap v_{2}\right|+\left|v_{1} \cap v_{3}\right|-t_{123} \leq\left|v_{1}\right|$ e se $\left|v_{1}\right|-\left(\left|v_{1} \cap v_{2}\right|+\left|v_{1} \cap v_{3}\right|-t_{123}\right)<8$;

4. Escolha $\left|v_{2} \cap v_{3}\right|$ de modo que $\left|v_{2} \cap v_{3}\right|-t_{123}<8$;

5. Calcule $\left|v_{1} \cap v_{2}\right|+\left|v_{2} \cap v_{3}\right|-t_{123}$ e $\left|v_{1} \cap v_{3}\right|+\left|v_{2} \cap v_{3}\right|-t_{123}$;

6. Determine os valores de $\left|v_{2}\right|$ e $\left|v_{3}\right|$ de modo que $\left|v_{2}\right|-\left(\left|v_{1} \cap v_{2}\right|+\left|v_{2} \cap v_{3}\right|-t_{123}\right)<8$ $\mathrm{e}\left|v_{3}\right|-\left(\left|v_{1} \cap v_{3}\right|+\left|v_{2} \cap v_{3}\right|-t_{123}\right)<8$.

Para facilitar os cálculos é necessário saber o comportamento dos geradores de cada representação. A partir dos vetores característicos dos loops correspondentes, obtemos a seguinte caracterização destes geradores: 


$\left.\begin{array}{c|c|l}i & \lambda\left(C_{i}^{3}\right) & \multicolumn{1}{|c}{\text { Caracterização dos geradores de } V_{i}^{3}} \\ \hline 1 & (1,1,1,1,1,1) & \left|v_{1}\right| \equiv\left|v_{2}\right| \equiv\left|v_{3}\right| \equiv 4(\bmod 8) \\ & & \left|v_{1} \cap v_{2}\right| \equiv\left|v_{1} \cap v_{3}\right| \equiv\left|v_{2} \cap v_{3}\right| \equiv 2(\bmod 4) \\ \hline 2 & (0,0,0,0,0,0) & \left|v_{1}\right| \equiv\left|v_{2}\right| \equiv\left|v_{3}\right| \equiv 0(\bmod 8) \\ & & \left|v_{1} \cap v_{2}\right| \equiv\left|v_{1} \cap v_{3}\right| \equiv\left|v_{2} \cap v_{3}\right| \equiv 0(\bmod 4) \\ \hline 3 & (0,0,0,1,1,1) & \left|v_{1}\right| \equiv\left|v_{2}\right| \equiv\left|v_{3}\right| \equiv 0(\bmod 8) \\ \hline 4 & (1,1,0,0,0,0) & \left|v_{1} \cap v_{2}\right| \equiv\left|v_{1} \cap v_{3}\right| \equiv\left|v_{2} \cap v_{3}\right| \equiv 2(\bmod 4) \\ \hline 5 & (1,0,0,0,0,0) & \left|v_{1} \cap v_{2}\right| \equiv\left|v_{1}\right| \equiv 4(\bmod 8) ;\left|v_{3}\right| \equiv 0(\bmod 8) ;\left|v_{3}\right| \equiv\left|v_{2} \cap v_{3}\right| \equiv 0(\bmod 4) \\ & & \left|v_{1} \cap v_{3}\right| \equiv 0(\bmod 8)\end{array}\right)$

\section{Ilustração}

Agora veremos uma ilustração de como encontrar algumas representações reduzidas associadas ao Loop de Código de posto 3 não-associativo $C_{4}^{3}$.

Para uma representação reduzida $V_{4}^{3}$ de um loop com vetor característico correspondente $(1,1,0,0,0,0)$, os elementos $v_{1}$ e $v_{2}$ terão pesos 12 ou 20, pois não é possível $\left|v_{1}\right|=4$ ou $\left|v_{2}\right|=4$, uma vez que $\left|v_{i} \cap v_{j}\right|$ é no mínimo 4 e não acontece $v_{i} \subseteq v_{j}, i \neq j$ e, para $\left|v_{1}\right|=28$, teríamos que ter classes de equivalência com 7 ou mais elementos. Se as 4 classes que compõem $v_{1}$ tivessem 7 elementos teríamos, por exemplo, $\left|v_{1} \cap v_{2}\right|=14$, o que é um absurdo, já que $\left|v_{i} \cap v_{j}\right|=4,8$, ou 12 . Temos também que $v_{3}$ tem pesos 8,16 ou 24 .

Suponha $\left|v_{1}\right|=12$, então $\left|v_{1} \cap v_{2}\right|=4$ ou 8 e $\left|v_{1} \cap v_{3}\right|=4$ ou 8. Nestes casos, $t_{123}=1$ ou 3. Notemos que $\left|v_{1} \cap v_{2}\right|-t_{123}<8,\left|v_{1} \cap v_{3}\right|-t_{123}<8,\left|v_{1} \cap v_{2}\right|+\left|v_{1} \cap v_{3}\right|-t_{123} \leq\left|v_{1}\right|$ e que $\left|v_{1}\right|-\left(\left|v_{1} \cap v_{2}\right|+\left|v_{1} \cap v_{3}\right|-t_{123}\right)<8$.

1. Suponha $\left|v_{1} \cap v_{2}\right|=4,\left|v_{1} \cap v_{3}\right|=4$ e $t_{123}=1$. Neste caso, $1<\left|v_{2} \cap v_{3}\right| \leq 8$.

Para $\left|v_{2} \cap v_{3}\right|=4$, temos $4<\left|v_{2}\right|<\left|v_{2} \cap v_{3}\right|+\left|v_{1} \cap v_{2}\right|-t_{123}+7$, ou seja, $\left|v_{2}\right|=12$. 
Analogamente, temos $\left|v_{3}\right|=8$.

Logo as classes de equivalência sobre $I_{m}$ são tais que $\left|X_{1}\right|=\left|X_{7}\right|=1$; $\left|X_{2}\right|=\left|X_{3}\right|=\left|X_{4}\right|=3 ;\left|X_{5}\right|=\left|X_{6}\right|=5$. Portanto, $m=21$ e o tipo de $V_{4}^{3}$ é dado por (1133355). A figura 3.1(a) ilustra este caso e assim, podemos supor $v_{1}=(1,2-4,5-7,8-12), v_{2}=(1,2-4,13-15,16-20)$ e $v_{3}=(1,5-7,13-15,21)$.

Para $\left|v_{2} \cap v_{3}\right|=8$, temos $\left|v_{2}\right|=12$ e $\left|v_{3}\right|=16$. Portanto $\left|X_{1}\right|=\left|X_{6}\right|=1 ;\left|X_{2}\right|=3$; $\left|X_{3}\right|=3 ;\left|X_{5}\right|=\left|X_{7}\right|=5,\left|X_{4}\right|=7$. Assim, $m=25$ e o tipo de $V_{4}^{3}$ é dado por (1133557). Podemos supor $v_{1}=(1,2-4,5-7,8-12), v_{2}=(1,2-4,13-19,20)$ e $v_{3}=(1,5-7,13-19,21-25)$ (Figura 3.1(b)).

Agora suponhamos $t_{123}=3$. Neste caso, $3<\left|v_{2} \cap v_{3}\right| \leq 10$.

Para $\left|v_{2} \cap v_{3}\right|=4:\left|v_{2}\right|=12,\left|v_{3}\right|=8$, grau 23 e tipo (1113377), como mostra a figura 3.1(c). Podemos supor $v_{1}=(1-3,4,5,6-12), v_{2}=(1-3,4,13,14-20)$ e $v_{3}=(1-3,5,13,21-23)$.

Para $\left|v_{2} \cap v_{3}\right|=8:\left|v_{2}\right|=12,\left|v_{3}\right|=16$, grau 27 e tipo (1133577), como mostra a figura 3.1(d). Podemos supor $v_{1}=(1-3,4,5,6-12), v_{2}=(1-3,4,13-17,18-20)$ e $v_{3}=(1-3,5,13-17,21-27)$.
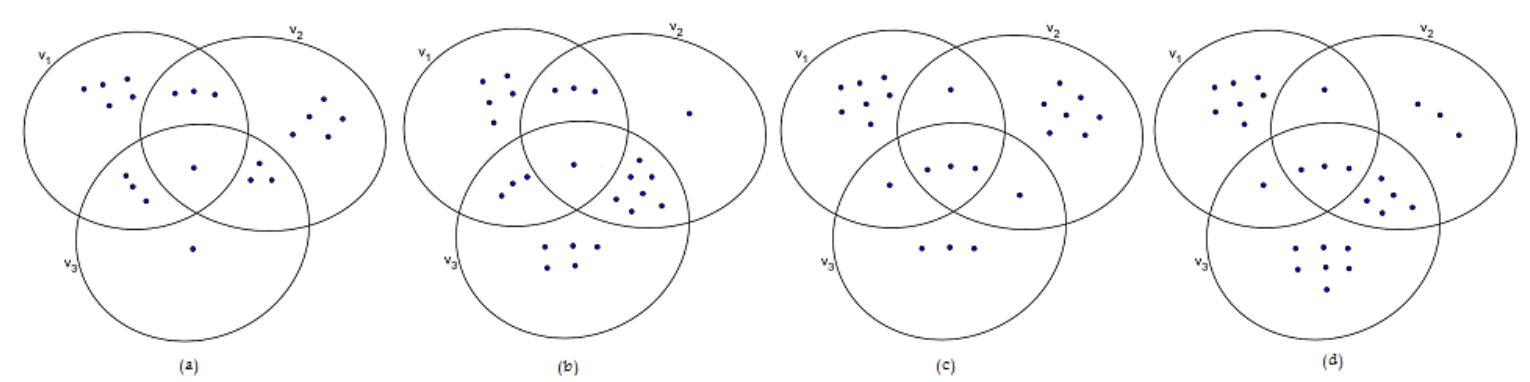

Figura 3.1: Representações de graus 21, 25, 23 e 27, respectivamente Nos próximos casos, os elementos de $v_{1}, v_{2}$ e $v_{3}$ são encontrados de maneira análoga ao que fizemos antes, ou seja, sabendo que o grau da representação é $m$ basta distribuirmos os números de 1 até $m$ observando pesos e intersecções correspondentes a estes geradores. 
2. Suponha $\left|v_{1} \cap v_{2}\right|=4,\left|v_{1} \cap v_{3}\right|=8$ e $t_{123}=1$.

Para $\left|v_{2} \cap v_{3}\right|=4:\left|v_{2}\right|=12,\left|v_{3}\right|=16$, grau 25 e tipo (1133557) (Figura 3.2(a)).

Para $\left|v_{2} \cap v_{3}\right|=8:\left|v_{2}\right|=12,\left|v_{3}\right|=16$, grau 21 e tipo (1111377) (Figura 3.2(b)).

Se $t_{123}=3$ :

Para $\left|v_{2} \cap v_{3}\right|=4:\left|v_{2}\right|=12,\left|v_{3}\right|=16$, grau 27 e tipo (1133577) (Figura 3.2(c)).

Para $\left|v_{2} \cap v_{3}\right|=8:\left|v_{2}\right|=12,\left|v_{3}\right|=16$, grau 23 e tipo (1333355) (Figura 3.2(d)).
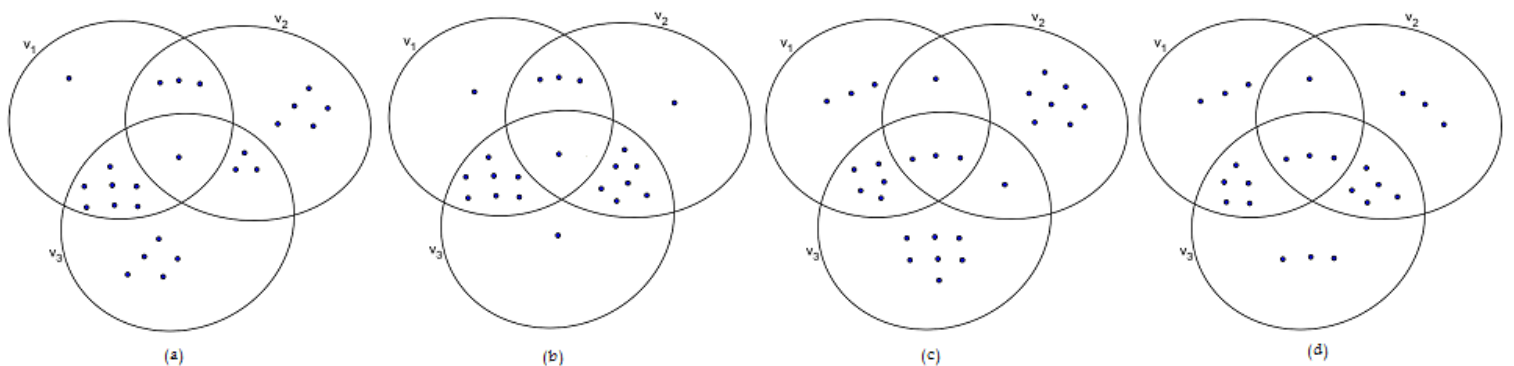

Figura 3.2: Representações de graus 25, 21, 27 e 23, respectivamente

3. Suponha $\left|v_{1} \cap v_{2}\right|=8,\left|v_{1} \cap v_{3}\right|=4$ e $t_{123}=1$.

Para $\left|v_{2} \cap v_{3}\right|=4:\left|v_{2}\right|=12,\left|v_{3}\right|=8$, grau 17 e tipo (1111337) (Figura 3.3(a)).

Para $\left|v_{2} \cap v_{3}\right|=8:\left|v_{2}\right|=20,\left|v_{3}\right|=16$, grau 29 e tipo (1135577) (Figura 3.3(b)).

Se $t_{123}=3$ :

Para $\left|v_{2} \cap v_{3}\right|=4:\left|v_{2}\right|=12,\left|v_{3}\right|=8$, grau 19 e tipo (1133335) (Figura 3.3(c)).

Para $\left|v_{2} \cap v_{3}\right|=8:\left|v_{2}\right|=20,\left|v_{3}\right|=16$, grau 31 e tipo (1335577) (Figura 3.3(d)).

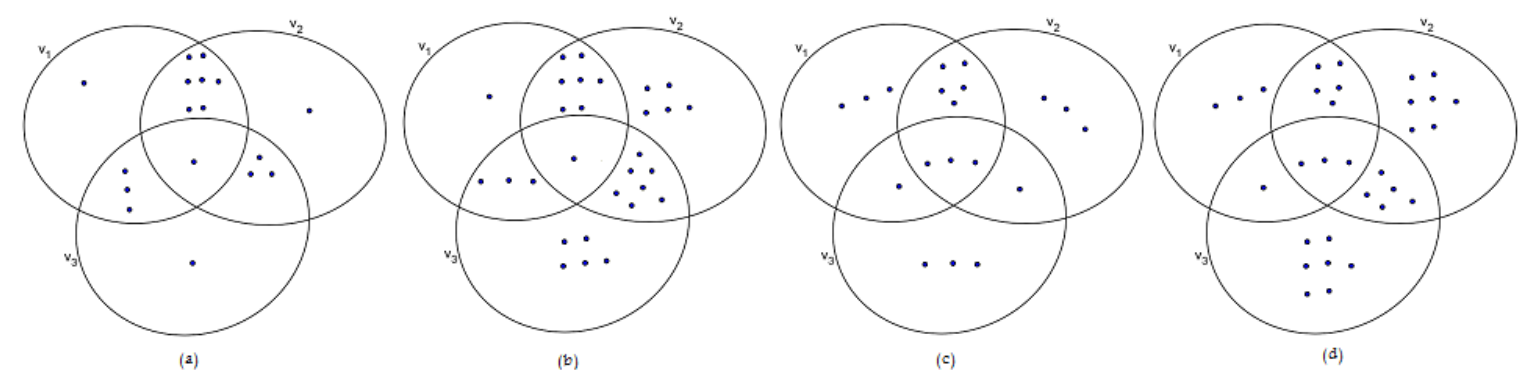

Figura 3.3: Representações de graus 17, 29, 19 e 31, respectivamente 
4. Suponha $\left|v_{1} \cap v_{2}\right|=8,\left|v_{1} \cap v_{3}\right|=8$ e $t_{123}=5$.

Para $\left|v_{2} \cap v_{3}\right|=8:\left|v_{2}\right|=12,\left|v_{3}\right|=16$, grau 21 e tipo (1133355) (Figura 3.4(a)).

Para $\left|v_{2} \cap v_{3}\right|=12:\left|v_{2}\right|=20,\left|v_{3}\right|=16$, grau 25 e tipo (1133557) (Figura 3.4(b)).

Se $t_{123}=7$ :

Para $\left|v_{2} \cap v_{3}\right|=8:\left|v_{2}\right|=12,\left|v_{3}\right|=16$, grau 23 e tipo (1113377) (Figura 3.4(c)).

Para $\left|v_{2} \cap v_{3}\right|=12:\left|v_{2}\right|=20,\left|v_{3}\right|=16$, grau 27 e tipo (1133577) (Figura 3.4(d)).
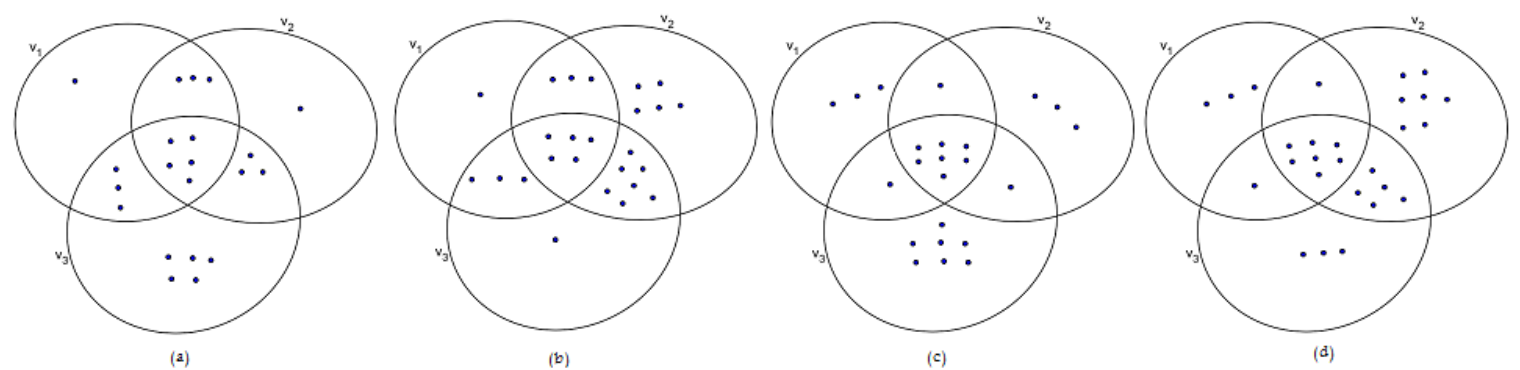

Figura 3.4: Representações de graus 21, 25, 23 e 27, respectivamente

Nesta ilustração, encontramos três representações de grau 21, das quais duas tem tipo (1133355) e a outra (1111377). Denotemos estas representações por $V_{41}^{3}, V_{42}^{3}$ e $V_{43}^{3}$, respectivamente, e suponhamos que $\left\{u_{1}, v_{1}, w_{1}\right\},\left\{u_{2}, v_{2}, w_{2}\right\}$ e $\left\{u_{3}, v_{3}, w_{3}\right\}$ sejam bases, respectivamente, de $V_{41}^{3}, V_{42}^{3}$ e $V_{43}^{3}$, dadas por:

$u_{1}=(1,2-4,5-7,8-12), v_{1}=(1,2-4,13-15,16-20), w_{1}=(1,5-7,13-15,21) ;$

$u_{2}=(1-8,9-11,12), v_{2}=(1-5,6-8,13-15,16), w_{2}=(1-5,9-11,13-15,17-21) ;$

$u_{3}=(1,2-4,5-11,12), v_{3}=(1,2-4,13-19,20), w_{3}=(1,5-11,13-19,21)$.

Afirmamos que $V_{41}^{3} \simeq V_{42}^{3}$ e que $V_{4 i}^{3} \not V_{43}^{3}, i=1,2$. De fato, seja $\varphi: I_{21} \longrightarrow I_{21}$ definida por

$1 \stackrel{\varphi}{\longmapsto} 12 ; 2 \stackrel{\varphi}{\longmapsto} 6 ; 3 \stackrel{\varphi}{\longmapsto} 7 ; 4 \stackrel{\varphi}{\longmapsto} 8 ; 5 \stackrel{\varphi}{\longmapsto} 9 ; 6 \stackrel{\varphi}{\longmapsto} 10 ; 7 \stackrel{\varphi}{\longmapsto} 11 ; 8 \stackrel{\varphi}{\longmapsto} 1 ; 9 \stackrel{\varphi}{\longmapsto} 2 ;$

$10 \stackrel{\varphi}{\longmapsto} 3 ; 11 \stackrel{\varphi}{\longmapsto} 4 ; 12 \stackrel{\varphi}{\longmapsto} 5 ; 16 \stackrel{\varphi}{\longmapsto} 21 ; 21 \stackrel{\varphi}{\longmapsto} 16 ; i \stackrel{\varphi}{\longmapsto} i, i=13,14,15,17, \ldots, 20$. 
Logo:

$$
\begin{aligned}
u_{1}^{\varphi} & =(1-12)=u_{2} \\
v_{1}^{\varphi} & =(6,7,8,12-15,17-21)=u_{2}+w_{2} \\
w_{1}^{\varphi} & =(9-16)=u_{2}+v_{2}
\end{aligned}
$$

Como $V_{42}^{3}=\left\langle u_{1}^{\varphi}, v_{1}^{\varphi}, w_{1}^{\varphi}\right\rangle$, temos $V_{41}^{3} \simeq V_{42}^{3}$.

Notemos que em ambas as representações isomorfas temos um elemento de ordem 8, cinco elementos de ordem 12 e um elemento de ordem 16.

Como existe um elemento de ordem 4 em $V_{43}^{3}$, a saber, $u_{3}+v_{3}+w_{3}=(1,12,20,21)$, concluímos que $V_{4 i}^{3}$ não é isomorfo a $V_{43}^{3}$, para $i=1,2$.

Procedendo de maneira análoga a ilustração feita, obtemos todas as representações reduzidas de posto 3. As Tabelas A.2-A.6 mostram todas estas representações.

\subsection{Representações básicas dos Loops de Código de posto 3}

Pretendemos nesta seção descrever todas as representações básicas dos loops de código de posto 3 .

Teorema 3.2.1. Os loops de código $C_{1}^{3}, \ldots, C_{5}^{3}$ tem as seguintes representações básicas $V_{1}, \ldots, V_{5}$, onde

$V_{1}=\langle(1234),(1256),(1357)\rangle$,

$V_{2}=\langle(12345678),(12349,10,11,12),(15679,10,11,13)\rangle$,

$V_{3}=\langle(12345678),(1234569,10),(1234579,11)\rangle$,

$V_{4}=\langle(1234),(1256789,10,11,12,13,14),(1356789,10,11,15,16,17)\rangle$,

$V_{5}=\langle(12345678910,11,12),(1-8,13,14,15,16),(1-5,9,10,11,13,14,15,17)\rangle$.

Demonstração. Considere os $\mathbf{F}_{2}$-subespaços $V_{1}, \ldots, V_{5}$ de $\mathbf{F}_{2}^{7}, \mathbf{F}_{2}^{13}, \mathbf{F}_{2}^{11}, \mathbf{F}_{2}^{17}, \mathbf{F}_{2}^{17}$, respectivamente, como acima. Primeiramente vejamos que cada espaço $V_{i}$ é uma repre- 
sentação de $C_{i}^{3}$, ou seja, é um código par de $\mathbf{F}_{2}^{m}$, para algum $m$ e que $C_{i}^{3} \simeq L\left(V_{i}\right)$, $i=1, \ldots, 5$.

Observando os elementos de $V_{5}$, ou seja, $v_{0}=0, v_{1}=(12345678910,11,12)$, $v_{2}=(1-8,13,14,15,16), v_{3}=(1-5,9,10,11,13,14,15,17), v_{4}=v_{1}+v_{2}=(9-16)$, $v_{5}=v_{1}+v_{3}=(6-8,12-15,17), v_{6}=v_{2}+v_{3}=(6-11,16,17)$ e $v_{7}=v_{1}+v_{2}+v_{3}=$ $(1-5,12,16,17)$, vemos claramente que todos os vetores tem peso múltiplo de 4 e o peso da intersecção dos vetores dois a dois é par. Assim, $V_{5}$ é código par. Analogamente, se prova que $V_{i}, i=1, \ldots, 4$ é código par.

Agora, o isomorfismo $C_{i}^{3} \simeq L\left(V_{i}\right)$ segue diretamente do Teorema 2.1.1 de Classificação de Loops de Código de posto 3 e da Proposição 2.1.1. Basta calcular o vetor característico associado a $L\left(V_{i}\right)$ e notar que ele pertece a órbita $O_{i}^{3}$ correspondente ao loop de código $C_{i}^{3}$.

Como exemplo, calculemos o vetor característico associado a $L\left(V_{5}\right)=\{1,-1\} \times V_{5}$. $\operatorname{Temos} v_{i}^{2}=(-1)^{\frac{\left|v_{i}\right|}{4}}=-1$ e $\left[v_{i}, v_{j}\right]=(-1)^{\frac{\left|v_{i} \cap v_{j}\right|}{2}}=1$, para $i, j=1,2,3$. Logo $\lambda\left(L\left(V_{5}\right)\right)=(111000)$ e $(111000) \in O_{5}^{3}$. Portanto, $C_{5}^{3} \simeq L\left(V_{5}\right)$.

Provaremos agora que cada $V_{i}, i=1, \ldots, 5$, a menos de isomorfismo, é a única representação básica do loop de código $C_{i}^{3}$. A menos que digamos o contrário, consideramos $X=(a, b, c)$ um conjunto de geradores para $C_{i}^{3}$ tal que $\lambda=\lambda_{X}\left(C_{i}^{3}\right)$ seja o vetor característico correspondente, e supomos que $V$ é uma representação básica de $C_{i}^{3}$, onde $v, w, u$ são os elementos da base de $V$ que correspondem a $a, b, c$. Usamos a notação $t=|v \cap w \cap u|$. Lembramos que $t \equiv 1(\bmod 2)$, pois $(a, b, c)=-1$.

Caso $i=1$ : Neste caso, estamos supondo $g r V \leq 7$. O vetor característico é $\lambda=(11111)$, então $|v| \equiv|w| \equiv|u| \equiv 4(\bmod 8)$. Suponha que $v=(1234)$, mas $[a, b]=-1$, então $|v \cap w| \equiv 2(\bmod 4) . \quad$ Logo $|v \cap w|=2$. Analogamente obtemos $|v \cap u|=2$.

Seja $w=(1256)$, daí obtemos também $|w \cap u|=2$ e assim, $t=1$. Logo, $u=(1357)$. Portanto, $V=V_{1}$. 
Caso $i=2$ : O vetor característico é $\lambda=(000000), \operatorname{logo}|v| \equiv|w| \equiv|u| \equiv 0(\bmod 8)$. Como gr $V \leq 13$, então $|v|=8$. Analogamente, $|w|=|u|=8$. Seja $v=(12345678)$. Como $|v \cap w| \equiv 0(\bmod 4), \operatorname{logo}|v \cap w|=4$. Analogamente, $|v \cap u|=|w \cap u|=4$.

Suponhamos $w=(12349,10,11,12)$. Temos duas possibilidades para $t: t=1$ ou $t=3$. Caso $t=3$, teremos gr $V=15$, contradição. Logo, $t=1$ e $u=$ $(15679,10,11,13)$. Portanto, $V=V_{2}$.

Caso $i=3$ : Consideramos $\lambda=(000111)$ e $g r V \leq 11$. Analogamente ao caso anterior, temos $|v|=|w|=|u|=8$. Neste caso, temos $|v \cap w|=|v \cap u|=|w \cap u|=6$. Suponhamos $v=(12345678)$ e $w=(1234569,10)$. Temos três possibilidades para $t$ : $t=1,3$ ou 5 . Caso $t \leq 3$ teremos $|v|>8$, contradição. Logo $t=5$ e podemos supor $u=(1234579,11)$. Portanto, $V=V_{3}$.

Caso $i=4$ : Neste caso, $\lambda=(111110)$. Como $|v| \equiv|w| \equiv|u| \equiv 4(\bmod 8) \mathrm{e}$ $|w \cap u| \equiv 0(\bmod 4)$ então $|w|=|u|=12$ e $|w \cap u|=8$. Seja $v=(1234)$. Então $|v \cap w|=2$. Se $|v \cap w|=4$, então $[v, w]=(-1)^{\frac{|v \cap w|}{2}}=1$, o que não acontece já que $[a, b]=-1$.

Seja $w=(1256789,10,11,12,13,14) . \quad$ Como $|v \cap w|=2$, então $t=1 . \quad$ Logo $u=(1356789,10,11,15,16,17)$. Portanto, $V=V_{4}$.

Agora suponhamos $v=(123456789,10,11,12)$. Se $|v \cap w| \leq 6$ então $g r V \geq 18$. De fato, temos $|v \cap w|=2,6$ ou 10. Se $|v \cap w|=2$ teríamos $|v+w|=20$, absurdo. Se $|v \cap w|=6$ teríamos $|v+w|=12$ e daí $g r V \geq 18$, absurdo. Portanto, $|v \cap w|=$ 10. Analogamente obtemos $|v \cap u|=10$. Sem perda de generalidade, suponhamos $w=(1256789,10,11,12,13,14)$. Como $t \equiv 1(\bmod 2)$, então as possibilidades para $t$ são 1,3,4 ou 7. Em qualquer caso teríamos $|v| \geq 13$, o que é uma contradição. Logo, não existe esta última possibilidade para $v$.

Caso $i=5$ : O vetor característico é $\lambda=(111000)$, então temos $|v| \equiv|w| \equiv$ $|u| \equiv 4(\bmod 8)$. Supondo $v=(1234)$ e como $[a, b]=1$, teremos $|v \cap w| \equiv 0(\bmod 4)$. 
Caso $v \cap w \neq \emptyset$ teremos $|v \cap w|=4$, e daí $v \subset w$, o que é uma contradição, pois isto nos dá $t \equiv 0(\bmod 2)$, o que não acontece. O caso $v \cap w=\emptyset$ também não acontece visto que devemos ter intersecção não-vazia entre $v, w$ e $u$.

Portanto $|v| \geq 12$. Analogamente provamos que $|w| \geq 12$ e $|u| \geq 12$.

Como a representação $V$ é básica e $g r V \leq 17$, obtemos $|v|=|w|=|u|=12$. Sem perda de generalidade, suponhamos $v=(123456789,10,11,12)$ e $|w \cap v|=4$ ou 8. Se $|v \cap w|=4$, então $|v+w|=16$ e daí $g r V \geq|v \cap w|+|v+w|=20$, o que é uma contradição.

Portanto, $v \cap w=(12345678)$ e $w=(12345678,13,14,15,16)$. Analogamente, temos $|v \cap u|=|w \cap u|=8$.

Se $t \leq 3$, então $|v| \geq|v \cap w \cap u|+|(v \cap w) \backslash(v \cap w \cap u)|+|(v \cap u) \backslash(v \cap w \cap u)| \geq$ $3+5+5=13$, contradição. Portanto, $t \geq 5$.

Se $t=7$, então $g r V \geq 19$. Para vermos isto considere $u=\left(i_{1}, \ldots, i_{12}\right)$, onde $1_{1}, \ldots, i_{7} \in v \cap w \cap u$, mas como $v \cap w=(12345678)$ então $i_{1}, \ldots, i_{7} \in v \cap w$. Suponha $u=$ $\left(1234567, i_{8}, \ldots, i_{12}\right)$. Como $|u \cap v|=8$ e $|u \cap w|=8$ então $i_{8} \in\{9,10,11,12\}$ enquanto que $i_{9} \in\{13,14,15,16\}$. Escolhamos $i_{8}=10$ e $i_{9}=13$. Daí $i_{j} \notin\{9,11,12,14,15,16\}$, $j=10,11,12$, pois seria $|u \cap v|>8$ ou $|u \cap w|>8$. Logo uma possibilidade para $u$ seria $u=(1234567,10,13,17,18,19)$ de modo que $g r V \geq 19$, contradição com $V$ ser representação básica. Assim, $t=5$ e $u=(123459,10,11,13,14,15,17)$. Portanto, $V=V_{5}$

Corolário 3.2.1. As representações básicas dos loops de códigos $C_{1}^{3}, \ldots, C_{5}^{3}$ tem os seguintes tipos:

$$
\text { (1111111), (1111333), (1111115), (1111337), (1113335). }
$$




\subsection{Representações básicas dos Loops de Código de posto 4}

De acordo com o Teorema 2.2.1, temos exatamente 16 loops de código de posto 4, a saber, $C_{1}^{4}, C_{2}^{4}, \ldots, C_{16}^{4}$. Para cada $C_{i}^{4}, i=1, \ldots, 16$, temos que encontrar $V_{i} \subseteq \mathbf{F}_{2}^{m}$ código par de grau $m$ minimal tal que $V_{i} \cong L\left(C_{i}^{4}\right)$.

De maneira geral, o conjunto $X=\{a, b, c, d\}$ representará um conjunto de geradores de $C_{i}^{4}$ tal que $\lambda_{X}\left(C_{i}^{4}\right)$ seja seu vetor característico correspondente.

Também suponhamos que $V_{i}=\operatorname{ger}\left\{v_{1}, v_{2}, v_{3}, v_{4}\right\}$ seja uma representação básica de $C_{i}^{4}$, onde $v_{1}, v_{2}, v_{3}, v_{4}$ correspondem a $a, b, c, d$ respectivamente.

Consideramos a notação: $t_{i j k}=\left|v_{i} \cap v_{j} \cap v_{k}\right|, i, j, k=1, . ., 4$ e $t_{1234}=\left|v_{1} \cap v_{2} \cap v_{3} \cap v_{4}\right|$.

Observemos que:

1. Para $\left|v_{i}\right| \equiv 0(\bmod 4)$ :

(a) Caso $v_{i}^{2}=-1$ temos $\left|v_{i}\right| \equiv 4(\bmod 8)$;

(b) Caso $v_{i}^{2}=1$ temos $\left|v_{i}\right| \equiv 0(\bmod 8)$.

2. Para $\left|v_{i} \cap v_{j}\right| \equiv 0(\bmod 2)$ :

(a) Caso $\left[v_{i}, v_{j}\right]=-1$ temos $\left|v_{i} \cap v_{j}\right| \equiv 2(\bmod 4)$;

(b) Caso $\left[v_{i}, v_{j}\right]=1$ temos $\left|v_{i} \cap v_{j}\right| \equiv 0(\bmod 4)$.

3. (a) Caso $t_{i j k}=-1$ temos $t_{i j k} \equiv 1(\bmod 2)$. Neste caso não acontece $v_{i} \subset v_{j}$, $v_{j} \subset v_{i}$ ou $v_{i} \cap v_{j}=\emptyset, i \neq j$.

(b) Caso $t_{i j k}=1$ temos $t_{i j k} \equiv 0(\bmod 2)$. 
Teorema 3.3.1. Os loops de código $C_{1}^{4}, \ldots, C_{16}^{4}$ tem as seguintes representações básicas $V_{1}, \ldots, V_{16}$ dadas por

$V_{1}=\langle(1234),(1256),(1357),(1-8)\rangle$,

$V_{2}=\langle(1-8),(1-4,9-12),(15679,10,11,13),(12589,12,13,14)\rangle$,

$V_{3}=\langle(1-8),(1-6,9,10),(1-5,7,9,11),(1,6-12)\rangle$,

$V_{4}=\langle(1-8),(1-6,9,10),(12379,11-17),(1478,9-11,18)\rangle$,

$V_{5}=\langle(1-8),(1234,9-12),(159,13-17),(12569,10,13,18)\rangle$,

$V_{6}=\langle(1234),(1256),(1357),(8,9,10,11)\rangle$,

$V_{7}=\langle(1-8),(1234,9-12),(15679,10,11,13),(14,15,16,17)\rangle$,

$V_{8}=\langle(1-8),(1234,9-12),(12359,13,14,15),(1,2,10,11,13,14,16,17)\rangle$,

$V_{9}=\langle(1-8),(1234,9-16),(15679,10,11,17),(5,6,9,10,12,13,18,19)\rangle$,

$V_{10}=\langle(1-8),(1,2,9-14),(139,10,11,15-17),(4,5,18,19)\rangle$,

$V_{11}=\langle(1-8),(1-4,9-12),(12359,13-15),(6,7,16,17)\rangle$,

$V_{12}=\langle(1-8),(1-4,9-12),(1235,9-11,13),(129,10,14-17)\rangle$,

$V_{13}=\langle(1-8),(129,10),(139,11),(45,12-17)\rangle$,

$V_{14}=\langle(1-8),(1-4,9-12),(15679,13-15),(2,3,10,11)\rangle$,

$V_{15}=\langle(1-12),(1-4,13-16),(1235,13-17),(1-6,13-18)\rangle$,

$V_{16}=\langle(1-8),(1,2,9-14),(1,3-7,9-13,15-19),(1-5,8,9,14-17,20)\rangle$.

Demonstração. Analogamente ao Teorema 3.2.1 (Representações dos loops de código de posto 3 ), se mostra que cada $V_{i}, i=1, \ldots, 16$, é um código par. Agora para mostrar que $V_{i} \simeq L\left(C_{i}^{4}\right)$ basta encontrar o vetor característico associado a $L\left(C_{i}^{4}\right)$ e aplicar o Teorema 2.2.1 (Classificação de loops de código de posto 4). Portanto, $V_{i}$ é uma representação de $C_{i}^{4}, i=1, \ldots, 16$.

Agora vamos mostrar, a menos de isomorfismo, que $V_{1}$ é a única representação básica de $C_{1}^{4}$. Considere $(a, b, c, d)$ um conjunto de geradores de $C_{1}^{4}$ tal que $\lambda=\lambda\left(C_{1}^{4}\right)=$ (1110110100). Suponhamos que $V=\operatorname{ger}\left\{v_{1}, v_{2}, v_{3}, v_{4}\right\}$ é uma representação básica de $C_{1}^{4}$, onde $v_{1}, v_{2}, v_{3}, v_{4}$ correspondem a $a, b, c, d$, respectivamente. Logo, $g r V \leq 8$. 
Neste caso, temos

$$
\begin{aligned}
\left|v_{1}\right| & \equiv\left|v_{2}\right| \equiv\left|v_{3}\right| \equiv 4(\bmod 8) \\
\left|v_{4}\right| & \equiv 0(\bmod 8) \\
\left|v_{1} \cap v_{2}\right| & \equiv\left|v_{1} \cap v_{3}\right| \equiv\left|v_{2} \cap v_{3}\right| \equiv 2(\bmod 4) \\
\left|v_{1} \cap v_{4}\right| & \equiv\left|v_{2} \cap v_{4}\right| \equiv\left|v_{3} \cap v_{4}\right| \equiv 0(\bmod 4)
\end{aligned}
$$

Suponha $v_{1}=(1234)$, então $\left|v_{1} \cap v_{2}\right|=\left|v_{1} \cap v_{3}\right|=2$ e daí, $t_{123}=1$. Logo podemos supor $v_{2}=(1256)$ e $v_{3}=(1357)$.

Analisaremos dois casos possíveis para valores de $t_{1234}$ : 0 e 1 .

Escreveremos sempre que necessário $t_{i j 4}$, para $\left|v_{i} \cap v_{j} \cap v_{4}\right| \operatorname{com} i, j=1,2,3, i \neq j$ e $t_{1234}$ para $\left|v_{1} \cap v_{2} \cap v_{3} \cap v_{4}\right|$.

Caso $t_{1234}=0$, teremos $t_{i j 4}=0$ e assim, $\left|v_{i} \cap v_{4}\right|=0$. Portanto, $\left|v_{4}\right| \geq 8$ e assim, não teremos representação reduzida básica neste caso. Caso $t_{1234}=1$, teremos $t_{i j 4}=2$ e assim, $\left|v_{i} \cap v_{4}\right|=4$, ou seja, $v_{i} \subset v_{4}, i=1,2,3$. Logo, $v_{4}=(12345678)$. Portanto, $V=V_{1}$.

Se $v_{1}=(123456789,10,11,12)$ teremos $g r V>12$, o que contradiz a hipótese de $V$ ser básica.

Agora provaremos que $V_{7}$, a menos de isomorfismo, é a única representação básica de $C_{7}^{4}$. Aqui o vetor característico é dado por $\lambda=(0001000000)$. Suponhamos que $V=\operatorname{ger}\left\{v_{1}, v_{2}, v_{3}, v_{4}\right\}$ é uma representação básica de $C_{7}^{4}$. Logo:

$$
\begin{aligned}
\left|v_{1}\right| & \equiv\left|v_{2}\right| \equiv\left|v_{3}\right| \equiv 0(\bmod 8) \\
\left|v_{4}\right| & \equiv 4(\bmod 8) \\
\left|v_{1} \cap v_{2}\right| & \equiv\left|v_{1} \cap v_{3}\right| \equiv\left|v_{2} \cap v_{3}\right| \equiv 0(\bmod 4) \\
\left|v_{1} \cap v_{4}\right| & \equiv\left|v_{2} \cap v_{4}\right| \equiv\left|v_{3} \cap v_{4}\right| \equiv 0(\bmod 4)
\end{aligned}
$$


Seja $v_{1}=(12345678)$, logo $\left|v_{1} \cap v_{2}\right|=\left|v_{1} \cap v_{3}\right|=4$ e assim, $t_{123}=1$ ou 3. Suponha $v_{2}=(12349,10,11,12)$, então $\left|v_{2} \cap v_{3}\right|=4$. Caso $t_{123}=1$, considere $v_{3}=(1567,9,10,11,13)$. Se $t_{1234}=0$, então $t_{i j 4}=0$ ou 2 . Tendo em vista que $\left|v_{i} \cap v_{j}\right| \equiv 0(\bmod 4), i, j=1,2,3, i \neq j$, então somente temos dois subcasos para analisar:

- $t_{i j 4}=0$ : Neste caso, $\left|v_{i} \cap v_{4}\right|=0$, para $i=1,2,3$ e assim, podemos supor $v_{4}=(14,15,16,17)$. Portanto, para este caso, $V=V_{7}$.

- $t_{i j 4}=2$ : Neste caso, $\left|v_{i} \cap v_{4}\right|=4$, para $i=1,2,3$ e assim, $v_{4}=(2,3,5,6,9,10,14-19)$, o que contradiz o grau minimal de $V$.

Se $t_{1234}=1$, então $t_{i j 4}=2$ ou 4 . Analogamente temos dois subcasos para analisar:

- $t_{i j 4}=2$ : Neste caso, $\left|v_{i} \cap v_{4}\right|=4$, para $i=1,2,3$, o que nos dá $g r V>17$, contradição.

- $t_{i j 4}=4$ : Neste caso, $\left|v_{i} \cap v_{4}\right|=8$, para $i=1,2,3$, que também contradiz o grau minimal de $V$.

Agora, analisando o caso $t_{123}=3$, suponha $v_{3}=(12359,13,14,15)$. Se $t_{1234}=0$, então $\left|v_{i} \cap v_{4}\right|=0$ e assim teremos $v_{4}=(16,17,18,19)$, o que contradiz o grau de $V$ ser minimal. Analogamente, para os casos $t_{1234}=1,2$ e 4 teremos $g r V>17$.

Considere agora o loop de código $C_{10}^{4} \operatorname{com} \lambda=(0001111000)$ e $V=\operatorname{ger}\left\{v_{1}, v_{2}, v_{3}, v_{4}\right\}$ uma representação básica. Seja $v_{1}=(12345678)$, então $\left|v_{1} \cap v_{2}\right|=2$ ou 6 e $\left|v_{1} \cap v_{3}\right|=2$ ou 6 .

Caso $\left|v_{1} \cap v_{2}\right|=2$, podemos supor $v_{2}=(1,2,9-14)$. Logo teremos $t_{123}=1 \mathrm{e}$ $\left|v_{2} \cap v_{3}\right|=4$.

- Para $\left|v_{1} \cap v_{3}\right|=2$, considere $v_{3}=(1,3,9,10,11,15,16,17)$. Se $t_{1234}=0$, então $t_{124}=t_{134}=0$ e $t_{234}=0$ ou 2. Logo $\left|v_{1} \cap v_{4}\right|=2$. Se $t_{234}=0:\left|v_{2} \cap v_{4}\right|=0 \mathrm{e}$ 
$\left|v_{3} \cap v_{4}\right|=0$. Logo teremos $v_{4}=(4,5,18,19)$ e, portanto, $V=V_{10}$. Já no caso $t_{234}=2$ encontraremos $v_{4}=(4,5,9,10,12,13,15,16,18-21)$, contradizendo a minimalidade do grau de $V$. A análise de $t_{1234}=1$ é análoga.

- Para $\left|v_{1} \cap v_{3}\right|=6$, podemos considerar $v_{3}=(1,3-7,9-11,15-21)$. Neste caso gr $V>19$ para qualquer análise.

Não teremos representação básica no caso $\left|v_{1} \cap v_{2}\right|=6$.

Analogamente, nos outros casos, prova-se que cada $V_{i}$ é representação básica única, a menos de isomorfismo.

Corolário 3.3.1. As representações básicas dos loops de código $C_{1}^{4}, \ldots, C_{16}^{4}$ tem os seguintes grau e tipo, respectivamente:

\begin{tabular}{c|c|l}
$i$ & $g r V_{i}$ & \multicolumn{1}{|c}{ tipo de $V_{i}$} \\
\hline 1 & 8 & $(1111111)$ \\
2 & 14 & $(1111111222)$ \\
3 & 12 & $(11111114)$ \\
4 & 18 & $(1111111226)$ \\
5 & 18 & $(11111112224)$ \\
6 & 11 & $(1111114)$ \\
7 & 17 & $(1111334)$ \\
8 & 17 & $(1111122223)$ \\
9 & 19 & $(1111222233)$ \\
10 & 19 & $(111223333)$ \\
11 & 17 & $(111122333)$ \\
12 & 17 & $(111112234)$ \\
13 & 17 & $(11111236)$ \\
14 & 15 & $(11112233)$ \\
15 & 18 & $(11111336)$ \\
16 & 20 & $(11111122334)$
\end{tabular}


Demonstração. O grau e tipo de cada representação, vem diretamente do teorema anterior.

\subsection{Considerações Finais}

Neste trabalho, estudamos loops de código e classificamos todos os loops de código não associativos de posto 3 e 4 . Encontramos todos os grupos de automorfismos externos destes loops com o auxílio do programa computacional Microsoft Office Excel. Além disso, determinamos todas as representações reduzidas dos loops de código não associativos de posto 3. Em particular, determinamos todas as representações básicas dos loops de código não associativos de posto 3 e 4 .

A partir dos resultados encontrados, poderemos também determinar todas as representações reduzidas dos loops de código não associativos de posto 4, classificar os loops de código não associativos de posto 5 e determinar as representações correspondentes destes loops, mas não serão estudados neste trabalho. Para determinar os grupos de automorfismos externos dos loops de código de posto 5, é fundamental a utilização do programa computacional "The GAP Group"(GAP - Groups, Algorithms and Programming).

Observemos nas Tabelas A.2-A.6 que, existem várias representações de loops de código de posto 3 com o mesmo grau e o mesmo tipo. Procedendo de maneira análoga à ilustração feita na seção 3.1, prova-se que estas representações são isomorfas. De maneira geral, temos a seguinte conjectura:

Conjectura 3.4.1. Sejam $V_{1}$ e $V_{2}$ representações de mesmo grau e mesmo tipo de um loop de código L. Então $V_{1}$ e $V_{2}$ são representações isomorfas. 
Apêndice A

\section{Tabelas}




\begin{tabular}{|c|c|c|c|c|c|}
\hline $\mathrm{n}$ & $(x, y, z)$ & $\lambda\left(C_{5}^{3}\right)$ & & & \\
\hline 1 & $(a, b, c)$ & $(100000)$ & & & \\
\hline 2 & $(c, a, b)$ & $(010000)$ & & & \\
\hline 3 & $(a b, a, c)$ & (110010) & $\mathrm{n}$ & $(x, y, z)$ & $\lambda\left(C_{4}^{3}\right)$ \\
\hline 4 & $(a b, c, a)$ & (101100) & 1 & $(a, b, c)$ & $\overline{(110000)}$ \\
\hline 5 & $(a, a b, c)$ & (110001) & 2 & $(a, c, b)$ & (101000) \\
\hline 6 & $(b c, a, b)$ & $(010100)$ & 3 & $(c, a, b)$ & $(011000)$ \\
\hline 7 & $(b c, b, a)$ & (001010) & 3 & $(c, a, b)$ & $\begin{array}{l}(011000) \\
(010010)\end{array}$ \\
\hline 8 & $(b, a, b c)$ & (010001) & 4 & $(a b, a, c)$ & (010010) \\
\hline 9 & $(a b c, a, b)$ & $(010110)$ & 5 & $(a b, c, a)$ & (001100) \\
\hline 10 & $(b, a b c, a)$ & (001101) & 6 & $(a, a b, c)$ & (100001) \\
\hline 11 & $(b, a, a b c)$ & $(010011)$ & 7 & $(b c, a, b)$ & (111100) \\
\hline 12 & $(a b, b c, a b c)$ & (100011) & 8 & $(b c, b, a)$ & (111010) \\
\hline 13 & $(a b, a b c, b c)$ & (100101) & 10 & $(b, a, b c)$ & (111001) \\
\hline 14 & $(a b c, b c, a b)$ & (001110) & 10 & $(b c, a, c)$ & (110100) \\
\hline 15 & $(b, c, a)$ & $(001000)$ & 11 & $(b c, c, a)$ & (101010) \\
\hline 16 & $(a, c, a b)$ & (101001) & 12 & $(c, a, b c)$ & (011001) \\
\hline 17 & $(a, b, b c)$ & $(100010)$ & 13 & $(a b . c, a, b)$ & (111110) \\
\hline 18 & $(c, b c, a)$ & (001001) & 14 & $(b, a b . c, a)$ & (111101) \\
\hline 19 & $(a, b c, c)$ & (100100) & 15 & $(b, a, a b . c)$ & (111011) \\
\hline 20 & $(b, a, a c)$ & (011010) & 16 & $(a b, b c, a b . c)$ & $(011011)$ \\
\hline 21 & $(b, a c, a)$ & (011100) & 17 & $(a b, a b . c, b c)$ & (011101) \\
\hline 22 & $(a b, a c, a)$ & $(111000)$ & 18 & $(b c, a b, a b . c)$ & (101011) \\
\hline 23 & $(a b, a c, a b c)$ & (110011) & 19 & $(b c, a b . c, a b)$ & (110101) \\
\hline 24 & $(a b, a b c, a c)$ & (101101) & 20 & $(a b . c, a b, b c)$ & (101110) \\
\hline 25 & $(a b c, a c, a b)$ & (011110) & 21 & $(a b c, b c, a b)$ & (110110) \\
\hline 26 & $(b c, b, a b c)$ & $(000011)$ & & & \\
\hline 27 & $(b c, a b c, b)$ & (000101) & & & \\
\hline 28 & $(a b c, b, b c)$ & $(000110)$ & & & \\
\hline
\end{tabular}

Tabela A.1: Vetores característicos das órbitas $O_{4}^{3}$ e $O_{5}^{3}$

As tabelas $A, B, C$ e $D$ abaixo tem a função de mostrar os quadrados e comutadores dos loops de código de posto 4 . 


\begin{tabular}{|c|c|c|c|c|c|c|c|c|c|c|c|c|c|c|c|c|}
\hline$A$ & $C_{2}^{4}$ & $C_{8}^{4}$ & $C_{12}^{4}$ & $C_{3}^{4}$ & $C_{7}^{4}$ & $C_{11}^{4}$ & $C_{14}^{4}$ & $C_{10}^{4}$ & $C_{16}^{4}$ & $C_{4}^{4}$ & $C_{9}^{4}$ & $C_{13}^{4}$ & $C_{15}^{4}$ & $C_{1}^{4}$ & $C_{6}^{4}$ & $C_{5}^{4}$ \\
\hline$a^{2}$ & 1 & 1 & 1 & 1 & 1 & 1 & 1 & 1 & 1 & 1 & 1 & 1 & -1 & -1 & -1 & 1 \\
\hline$b^{2}$ & 1 & 1 & 1 & 1 & 1 & 1 & 1 & 1 & 1 & 1 & -1 & -1 & 1 & -1 & -1 & 1 \\
\hline$c^{2}$ & 1 & 1 & 1 & 1 & 1 & 1 & 1 & 1 & 1 & -1 & 1 & -1 & 1 & -1 & -1 & 1 \\
\hline$d^{2}$ & 1 & 1 & 1 & 1 & -1 & -1 & -1 & -1 & -1 & 1 & 1 & 1 & -1 & 1 & -1 & 1 \\
\hline$[a, b]$ & 1 & 1 & 1 & -1 & 1 & 1 & 1 & -1 & -1 & -1 & 1 & -1 & 1 & -1 & -1 & 1 \\
\hline$[a, c]$ & 1 & 1 & 1 & -1 & 1 & 1 & 1 & -1 & -1 & 1 & 1 & -1 & 1 & -1 & -1 & -1 \\
\hline$[a, d]$ & 1 & -1 & -1 & 1 & 1 & -1 & -1 & -1 & -1 & 1 & -1 & -1 & -1 & 1 & 1 & 1 \\
\hline$[b, c]$ & 1 & 1 & -1 & -1 & 1 & 1 & -1 & 1 & -1 & 1 & 1 & -1 & -1 & -1 & -1 & -1 \\
\hline$[b, d]$ & 1 & 1 & 1 & 1 & 1 & 1 & 1 & 1 & 1 & 1 & 1 & 1 & 1 & 1 & 1 & 1 \\
\hline$[c, d]$ & 1 & 1 & 1 & 1 & 1 & 1 & 1 & 1 & 1 & 1 & 1 & 1 & 1 & 1 & 1 & 1 \\
\hline$(a b)^{2}$ & 1 & 1 & 1 & -1 & 1 & 1 & 1 & -1 & -1 & -1 & -1 & 1 & -1 & -1 & -1 & 1 \\
\hline$(a c)^{2}$ & 1 & 1 & 1 & -1 & 1 & 1 & 1 & -1 & -1 & -1 & 1 & 1 & -1 & -1 & -1 & -1 \\
\hline$(b c)^{2}$ & 1 & 1 & -1 & -1 & 1 & 1 & -1 & 1 & -1 & -1 & -1 & -1 & -1 & -1 & -1 & -1 \\
\hline$(a d)^{2}$ & 1 & -1 & -1 & 1 & -1 & 1 & 1 & 1 & 1 & 1 & -1 & -1 & -1 & -1 & 1 & 1 \\
\hline$(b d)^{2}$ & 1 & 1 & 1 & 1 & -1 & -1 & -1 & -1 & -1 & 1 & -1 & -1 & -1 & -1 & 1 & 1 \\
\hline$(c d)^{2}$ & 1 & 1 & 1 & 1 & -1 & -1 & -1 & -1 & -1 & -1 & 1 & -1 & -1 & -1 & 1 & 1 \\
\hline$(a b c)^{2}$ & -1 & -1 & 1 & 1 & -1 & -1 & 1 & -1 & 1 & -1 & 1 & 1 & -1 & -1 & -1 & -1 \\
\hline$(a b d)^{2}$ & 1 & -1 & -1 & -1 & -1 & 1 & 1 & -1 & -1 & -1 & 1 & -1 & -1 & -1 & 1 & 1 \\
\hline$(a c d)^{2}$ & 1 & -1 & -1 & -1 & -1 & 1 & 1 & -1 & -1 & -1 & -1 & -1 & -1 & -1 & 1 & -1 \\
\hline$(b c d)^{2}$ & 1 & 1 & -1 & -1 & -1 & -1 & 1 & -1 & 1 & -1 & -1 & -1 & 1 & -1 & 1 & -1 \\
\hline$(a b c d)^{2}$ & -1 & 1 & -1 & 1 & 1 & -1 & 1 & -1 & 1 & -1 & -1 & -1 & -1 & -1 & 1 & -1 \\
\hline$[a, a b]$ & 1 & 1 & 1 & -1 & 1 & 1 & 1 & -1 & -1 & -1 & 1 & -1 & 1 & -1 & -1 & 1 \\
\hline$[a, a c]$ & 1 & 1 & 1 & -1 & 1 & 1 & 1 & -1 & -1 & 1 & 1 & -1 & 1 & -1 & -1 & -1 \\
\hline$[a, a d]$ & 1 & -1 & -1 & 1 & 1 & -1 & -1 & -1 & -1 & 1 & -1 & -1 & -1 & 1 & 1 & 1 \\
\hline$[a, b c]$ & -1 & -1 & -1 & -1 & -1 & -1 & -1 & -1 & -1 & 1 & -1 & -1 & -1 & -1 & -1 & 1 \\
\hline$[a, b d]$ & 1 & -1 & -1 & -1 & 1 & -1 & -1 & 1 & 1 & -1 & -1 & 1 & -1 & -1 & -1 & 1 \\
\hline$[a, c d]$ & 1 & -1 & -1 & -1 & 1 & -1 & -1 & 1 & 1 & 1 & -1 & 1 & -1 & -1 & -1 & -1 \\
\hline
\end{tabular}




\begin{tabular}{|c|c|c|c|c|c|c|c|c|c|c|c|c|c|c|c|c|}
\hline$B$ & $C_{2}^{4}$ & $C_{8}^{4}$ & $C_{12}^{4}$ & $C_{3}^{4}$ & $C_{7}^{4}$ & $C_{11}^{4}$ & $C_{14}^{4}$ & $C_{10}^{4}$ & $C_{16}^{4}$ & $C_{4}^{4}$ & $C_{9}^{4}$ & $C_{13}^{4}$ & $C_{15}^{4}$ & $C_{1}^{4}$ & $C_{6}^{4}$ & $C_{5}^{4}$ \\
\hline$[a, a b c]$ & -1 & -1 & -1 & -1 & -1 & -1 & -1 & -1 & -1 & 1 & -1 & -1 & -1 & -1 & -1 & 1 \\
\hline$[a, a b d]$ & 1 & -1 & -1 & -1 & 1 & -1 & -1 & 1 & 1 & -1 & -1 & 1 & -1 & -1 & -1 & 1 \\
\hline$[a, a c d]$ & 1 & -1 & -1 & -1 & 1 & -1 & -1 & 1 & 1 & 1 & -1 & 1 & -1 & -1 & -1 & -1 \\
\hline$[a, b c d]$ & -1 & 1 & 1 & -1 & -1 & 1 & 1 & 1 & 1 & 1 & 1 & 1 & 1 & -1 & -1 & 1 \\
\hline$[a, a b c d]$ & -1 & 1 & 1 & -1 & -1 & 1 & 1 & 1 & 1 & 1 & 1 & 1 & 1 & -1 & -1 & 1 \\
\hline$[b, a b]$ & 1 & 1 & 1 & -1 & 1 & 1 & 1 & -1 & -1 & -1 & 1 & -1 & 1 & -1 & -1 & 1 \\
\hline$[b, a c]$ & -1 & -1 & 1 & -1 & -1 & -1 & 1 & 1 & -1 & 1 & -1 & -1 & 1 & -1 & -1 & 1 \\
\hline$[b, a d]$ & 1 & 1 & 1 & -1 & 1 & 1 & 1 & -1 & -1 & -1 & 1 & -1 & 1 & -1 & -1 & 1 \\
\hline$[b, b c]$ & 1 & 1 & -1 & -1 & 1 & 1 & -1 & 1 & -1 & 1 & 1 & -1 & -1 & -1 & -1 & -1 \\
\hline$[b, b d]$ & 1 & 1 & 1 & 1 & 1 & 1 & 1 & 1 & 1 & 1 & 1 & 1 & 1 & 1 & 1 & 1 \\
\hline$[b, c d]$ & 1 & 1 & -1 & -1 & 1 & 1 & -1 & 1 & -1 & 1 & 1 & -1 & -1 & -1 & -1 & -1 \\
\hline$[b, a b c]$ & -1 & -1 & 1 & -1 & -1 & -1 & 1 & 1 & -1 & 1 & -1 & -1 & 1 & -1 & -1 & 1 \\
\hline$[b, a b d]$ & 1 & 1 & 1 & -1 & 1 & 1 & 1 & -1 & -1 & -1 & 1 & -1 & 1 & -1 & -1 & 1 \\
\hline$[b, a c d]$ & -1 & -1 & 1 & -1 & -1 & -1 & 1 & 1 & -1 & 1 & -1 & -1 & 1 & -1 & -1 & 1 \\
\hline$[b, b c d]$ & 1 & 1 & -1 & -1 & 1 & 1 & -1 & 1 & -1 & 1 & 1 & -1 & -1 & -1 & -1 & -1 \\
\hline$[b, a b c d]$ & -1 & -1 & 1 & -1 & -1 & -1 & 1 & 1 & -1 & 1 & -1 & -1 & 1 & -1 & -1 & 1 \\
\hline$[c, a b]$ & -1 & -1 & 1 & -1 & -1 & -1 & 1 & 1 & -1 & -1 & -1 & -1 & 1 & -1 & -1 & -1 \\
\hline$[c, a c]$ & 1 & 1 & 1 & -1 & 1 & 1 & 1 & -1 & -1 & 1 & 1 & -1 & 1 & -1 & -1 & -1 \\
\hline$[c, a d]$ & 1 & 1 & 1 & -1 & 1 & 1 & 1 & -1 & -1 & 1 & 1 & -1 & 1 & -1 & -1 & -1 \\
\hline$[c, b c]$ & 1 & 1 & -1 & -1 & 1 & 1 & -1 & 1 & -1 & 1 & 1 & -1 & -1 & -1 & -1 & -1 \\
\hline$[c, b d]$ & 1 & 1 & -1 & -1 & 1 & 1 & -1 & 1 & -1 & 1 & 1 & -1 & -1 & -1 & -1 & -1 \\
\hline$[c, c d]$ & 1 & 1 & 1 & 1 & 1 & 1 & 1 & 1 & 1 & 1 & 1 & 1 & 1 & 1 & 1 & 1 \\
\hline$[c, a b c]$ & -1 & -1 & 1 & -1 & -1 & -1 & 1 & 1 & -1 & -1 & -1 & -1 & 1 & -1 & -1 & -1 \\
\hline$[c, a b d]$ & -1 & -1 & 1 & -1 & -1 & -1 & 1 & 1 & -1 & -1 & -1 & -1 & 1 & -1 & -1 & -1 \\
\hline$[c, a c d]$ & 1 & 1 & 1 & -1 & 1 & 1 & 1 & -1 & -1 & 1 & 1 & -1 & 1 & -1 & -1 & -1 \\
\hline$[c, b c d]$ & 1 & 1 & -1 & -1 & 1 & 1 & -1 & 1 & -1 & 1 & 1 & -1 & -1 & -1 & -1 & -1 \\
\hline$[c, a b c d]$ & -1 & -1 & 1 & -1 & -1 & -1 & 1 & 1 & -1 & -1 & -1 & -1 & 1 & -1 & -1 & -1 \\
\hline$[d, a b]$ & 1 & -1 & -1 & 1 & 1 & -1 & -1 & -1 & -1 & 1 & -1 & -1 & -1 & 1 & 1 & 1 \\
\hline$[d, a c]$ & 1 & -1 & -1 & 1 & 1 & -1 & -1 & -1 & -1 & 1 & -1 & -1 & -1 & 1 & 1 & 1 \\
\hline$[d, a d]$ & 1 & -1 & -1 & 1 & 1 & -1 & -1 & -1 & -1 & 1 & -1 & -1 & -1 & 1 & 1 & 1 \\
\hline$[d, b c]$ & 1 & 1 & 1 & 1 & 1 & 1 & 1 & 1 & 1 & 1 & 1 & 1 & 1 & 1 & 1 & 1 \\
\hline$[d, b d]$ & 1 & 1 & 1 & 1 & 1 & 1 & 1 & 1 & 1 & 1 & 1 & 1 & 1 & 1 & 1 & 1 \\
\hline$[d, c d]$ & 1 & 1 & 1 & 1 & 1 & 1 & 1 & 1 & 1 & 1 & 1 & 1 & 1 & 1 & 1 & 1 \\
\hline$[d, a b c]$ & 1 & -1 & -1 & 1 & 1 & -1 & -1 & -1 & -1 & 1 & -1 & -1 & -1 & 1 & 1 & 1 \\
\hline$[d, a b d]$ & 1 & -1 & -1 & 1 & 1 & -1 & -1 & -1 & -1 & 1 & -1 & -1 & -1 & 1 & 1 & 1 \\
\hline$[d, a c d]$ & 1 & -1 & -1 & 1 & 1 & -1 & -1 & -1 & -1 & 1 & -1 & -1 & -1 & 1 & 1 & 1 \\
\hline
\end{tabular}




\begin{tabular}{|c|c|c|c|c|c|c|c|c|c|c|c|c|c|c|c|c|}
\hline$C$ & $C_{2}^{4}$ & $C_{8}^{4}$ & $C_{12}^{4}$ & $C_{3}^{4}$ & $C_{7}^{4}$ & $C_{11}^{4}$ & $C_{14}^{4}$ & $C_{10}^{4}$ & $C_{16}^{4}$ & $C_{4}^{4}$ & $C_{9}^{4}$ & $C_{13}^{4}$ & $C_{15}^{4}$ & $C_{1}^{4}$ & $C_{6}^{4}$ & $C_{5}^{4}$ \\
\hline$[d, b c d]$ & 1 & 1 & 1 & 1 & 1 & 1 & 1 & 1 & 1 & 1 & 1 & 1 & 1 & 1 & 1 & 1 \\
\hline$[d, a b c d]$ & 1 & -1 & -1 & 1 & 1 & -1 & -1 & -1 & -1 & 1 & -1 & -1 & -1 & 1 & 1 & 1 \\
\hline$[a b, a c]$ & 1 & 1 & -1 & -1 & 1 & 1 & -1 & 1 & -1 & -1 & 1 & -1 & -1 & -1 & -1 & 1 \\
\hline$[a b, a d]$ & 1 & -1 & -1 & -1 & 1 & -1 & -1 & 1 & 1 & -1 & -1 & 1 & -1 & -1 & -1 & 1 \\
\hline$[a b, b c]$ & 1 & 1 & -1 & -1 & 1 & 1 & -1 & 1 & -1 & -1 & 1 & -1 & -1 & -1 & -1 & 1 \\
\hline$[a b, b d]$ & 1 & -1 & -1 & -1 & 1 & -1 & -1 & 1 & 1 & -1 & -1 & 1 & -1 & -1 & -1 & 1 \\
\hline$[a b, c d]$ & -1 & 1 & -1 & -1 & -1 & 1 & -1 & -1 & 1 & -1 & 1 & 1 & -1 & -1 & -1 & -1 \\
\hline$[a b, a b c]$ & -1 & -1 & 1 & -1 & -1 & -1 & 1 & 1 & -1 & -1 & -1 & -1 & 1 & -1 & -1 & -1 \\
\hline$[a b, a b d]$ & 1 & -1 & -1 & 1 & 1 & -1 & -1 & -1 & -1 & 1 & -1 & -1 & -1 & 1 & 1 & 1 \\
\hline$[a b, a c d]$ & 1 & -1 & 1 & -1 & 1 & -1 & 1 & -1 & 1 & -1 & -1 & 1 & 1 & -1 & -1 & 1 \\
\hline$[a b, b c d]$ & 1 & -1 & 1 & -1 & 1 & -1 & 1 & -1 & 1 & -1 & -1 & 1 & 1 & -1 & -1 & 1 \\
\hline$[a b, a b c d]$ & -1 & 1 & -1 & -1 & -1 & 1 & -1 & -1 & 1 & -1 & -1 & 1 & -1 & -1 & -1 & -1 \\
\hline$[a c, a d]$ & 1 & -1 & -1 & -1 & 1 & -1 & -1 & 1 & 1 & 1 & -1 & 1 & -1 & -1 & -1 & -1 \\
\hline$[a c, b c]$ & 1 & 1 & -1 & -1 & 1 & 1 & -1 & 1 & -1 & -1 & 1 & -1 & -1 & -1 & -1 & 1 \\
\hline$[a c, b d]$ & -1 & 1 & -1 & -1 & -1 & 1 & -1 & -1 & 1 & 1 & 1 & 1 & -1 & -1 & -1 & 1 \\
\hline$[a c, c d]$ & 1 & -1 & -1 & -1 & 1 & -1 & -1 & 1 & 1 & 1 & -1 & 1 & -1 & -1 & -1 & -1 \\
\hline$[a c, a b c]$ & -1 & -1 & 1 & -1 & -1 & -1 & 1 & 1 & -1 & 1 & -1 & -1 & 1 & -1 & -1 & 1 \\
\hline$[a c, a b d]$ & 1 & -1 & 1 & -1 & 1 & -1 & 1 & -1 & 1 & -1 & -1 & 1 & 1 & -1 & -1 & 1 \\
\hline$[a c, a c d]$ & 1 & -1 & -1 & 1 & 1 & -1 & -1 & -1 & -1 & 1 & -1 & -1 & -1 & 1 & 1 & 1 \\
\hline$[a c, b c d]$ & 1 & -1 & 1 & -1 & 1 & -1 & 1 & -1 & 1 & -1 & -1 & 1 & 1 & -1 & -1 & 1 \\
\hline$[a c, a b c d]$ & -1 & 1 & -1 & -1 & -1 & 1 & -1 & -1 & 1 & 1 & 1 & 1 & -1 & -1 & -1 & 1 \\
\hline$[a d, b c]$ & -1 & -1 & -1 & -1 & -1 & -1 & -1 & -1 & -1 & 1 & -1 & -1 & -1 & -1 & -1 & 1 \\
\hline$[a d, b d]$ & 1 & -1 & -1 & -1 & 1 & -1 & -1 & 1 & 1 & -1 & -1 & 1 & -1 & -1 & -1 & 1 \\
\hline$[a d, c d]$ & 1 & -1 & -1 & -1 & 1 & -1 & -1 & 1 & 1 & 1 & -1 & 1 & -1 & -1 & -1 & -1 \\
\hline$[a d, a b c]$ & -1 & 1 & 1 & -1 & -1 & 1 & 1 & 1 & 1 & 1 & 1 & 1 & 1 & -1 & -1 & 1 \\
\hline
\end{tabular}




\begin{tabular}{|c|c|c|c|c|c|c|c|c|c|c|c|c|c|c|c|c|}
\hline$D$ & $C_{2}^{4}$ & $C_{8}^{4}$ & $C_{12}^{4}$ & $C_{3}^{4}$ & $C_{7}^{4}$ & $C_{11}^{4}$ & $C_{14}^{4}$ & $C_{10}^{4}$ & $C_{16}^{4}$ & $C_{4}^{4}$ & $C_{9}^{4}$ & $C_{13}^{4}$ & $C_{15}^{4}$ & $C_{1}^{4}$ & $C_{6}^{4}$ & $C_{5}^{4}$ \\
\hline$[a d, a b d]$ & 1 & 1 & 1 & -1 & 1 & 1 & 1 & -1 & -1 & -1 & 1 & -1 & 1 & -1 & -1 & 1 \\
\hline$[a d, a c d]$ & 1 & 1 & 1 & -1 & 1 & 1 & 1 & -1 & -1 & 1 & 1 & -1 & 1 & -1 & -1 & -1 \\
\hline$[a d, b c d]$ & -1 & 1 & 1 & -1 & -1 & 1 & 1 & 1 & 1 & 1 & 1 & 1 & 1 & -1 & -1 & 1 \\
\hline$[a d, a b c d]$ & -1 & -1 & -1 & -1 & -1 & -1 & -1 & -1 & -1 & 1 & -1 & -1 & -1 & -1 & -1 & 1 \\
\hline$[b c, b d]$ & 1 & 1 & -1 & -1 & 1 & 1 & -1 & 1 & -1 & 1 & 1 & -1 & -1 & -1 & -1 & -1 \\
\hline$[b c, c d]$ & 1 & 1 & -1 & -1 & 1 & 1 & -1 & 1 & -1 & 1 & 1 & -1 & -1 & -1 & -1 & -1 \\
\hline$[b c, a b c]$ & -1 & -1 & -1 & -1 & -1 & -1 & -1 & -1 & -1 & 1 & -1 & -1 & -1 & -1 & -1 & 1 \\
\hline$[b c, a b d]$ & 1 & 1 & -1 & -1 & 1 & 1 & -1 & 1 & -1 & -1 & 1 & -1 & -1 & -1 & -1 & 1 \\
\hline$[b c, a c d]$ & 1 & 1 & -1 & -1 & 1 & 1 & -1 & 1 & -1 & -1 & 1 & -1 & -1 & -1 & -1 & 1 \\
\hline$[b c, b c d]$ & 1 & 1 & 1 & 1 & 1 & 1 & 1 & 1 & 1 & 1 & 1 & 1 & 1 & 1 & 1 & 1 \\
\hline$[b c, a b c d]$ & -1 & -1 & -1 & -1 & -1 & -1 & -1 & -1 & -1 & 1 & -1 & -1 & -1 & -1 & -1 & 1 \\
\hline$[b d, c d]$ & 1 & 1 & -1 & -1 & 1 & 1 & -1 & 1 & -1 & 1 & 1 & -1 & -1 & -1 & -1 & -1 \\
\hline$[b d, a b c]$ & -1 & 1 & -1 & -1 & -1 & 1 & -1 & -1 & 1 & 1 & 1 & 1 & -1 & -1 & -1 & 1 \\
\hline$[b d, a b d]$ & 1 & -1 & -1 & -1 & 1 & -1 & -1 & 1 & 1 & -1 & -1 & 1 & -1 & -1 & -1 & 1 \\
\hline$[b d, a c d]$ & -1 & 1 & -1 & -1 & -1 & 1 & -1 & -1 & 1 & 1 & 1 & 1 & -1 & -1 & -1 & 1 \\
\hline$[b d, b c d]$ & 1 & 1 & -1 & -1 & 1 & 1 & -1 & 1 & -1 & 1 & 1 & -1 & -1 & -1 & -1 & -1 \\
\hline$[b d, a b c d]$ & -1 & 1 & -1 & -1 & -1 & 1 & -1 & -1 & 1 & 1 & 1 & 1 & -1 & -1 & -1 & 1 \\
\hline$[c d, a b c]$ & -1 & 1 & -1 & -1 & -1 & 1 & -1 & -1 & 1 & -1 & 1 & 1 & -1 & -1 & -1 & -1 \\
\hline$[c d, a b d]$ & -1 & 1 & -1 & -1 & -1 & 1 & -1 & -1 & 1 & -1 & 1 & 1 & -1 & -1 & -1 & -1 \\
\hline$[c d, a c d]$ & 1 & -1 & -1 & -1 & 1 & -1 & -1 & 1 & 1 & 1 & -1 & 1 & -1 & -1 & -1 & -1 \\
\hline$[c d, b c d]$ & 1 & 1 & -1 & -1 & 1 & 1 & -1 & 1 & -1 & 1 & 1 & -1 & -1 & -1 & -1 & -1 \\
\hline$[c d, a b c d]$ & -1 & 1 & -1 & -1 & -1 & 1 & -1 & -1 & 1 & -1 & 1 & 1 & -1 & -1 & -1 & -1 \\
\hline$[a b c, a b d]$ & -1 & 1 & -1 & -1 & -1 & 1 & -1 & -1 & 1 & -1 & 1 & 1 & -1 & -1 & -1 & -1 \\
\hline$[a b c, a c d]$ & -1 & 1 & -1 & -1 & -1 & 1 & -1 & -1 & 1 & 1 & 1 & 1 & -1 & -1 & -1 & 1 \\
\hline$[a b c, b c d]$ & -1 & 1 & 1 & -1 & -1 & 1 & 1 & 1 & 1 & 1 & 1 & 1 & 1 & -1 & -1 & 1 \\
\hline$[a b c, a b c d]$ & 1 & -1 & -1 & 1 & 1 & -1 & -1 & -1 & -1 & 1 & -1 & -1 & -1 & 1 & 1 & 1 \\
\hline$[a b d, a c d]$ & 1 & 1 & -1 & -1 & 1 & 1 & -1 & 1 & -1 & -1 & 1 & -1 & -1 & -1 & -1 & 1 \\
\hline$[a b d, b c d]$ & 1 & -1 & 1 & -1 & 1 & -1 & 1 & -1 & 1 & -1 & -1 & 1 & 1 & -1 & -1 & 1 \\
\hline$[a b d, a b c d]$ & -1 & -1 & 1 & -1 & -1 & -1 & 1 & 1 & -1 & -1 & -1 & -1 & 1 & -1 & -1 & -1 \\
\hline$[a c d, b c d]$ & 1 & -1 & 1 & -1 & 1 & -1 & 1 & -1 & 1 & -1 & -1 & 1 & 1 & -1 & -1 & 1 \\
\hline$[a c d, a b c d]$ & -1 & -1 & 1 & -1 & -1 & -1 & 1 & 1 & -1 & 1 & -1 & -1 & 1 & -1 & -1 & 1 \\
\hline$[b c d, a b c d]$ & -1 & 1 & 1 & -1 & -1 & 1 & 1 & 1 & 1 & 1 & 1 & 1 & 1 & -1 & -1 & 1 \\
\hline
\end{tabular}




\begin{tabular}{|c|c|c|c|c|c|c|c|c|}
\hline$t_{123}$ & $\mid v_{1} \cap v_{2}$ & $\left|v_{1} \cap v_{3}\right|$ & $\mid v_{1}$ & $\left|v_{2} \cap v_{3}\right|$ & $\left|v_{2}\right|$ & $\left|v_{3}\right|$ & grau & tipo \\
\hline \multirow{8}{*}{1} & \multirow{4}{*}{2} & 2 & 4 & 2 & 4 & 4 & 7 & 1111111 \\
\hline & & & & 6 & 12 & 12 & 19 & 1111555 \\
\hline & & 6 & 12 & 2 & 4 & 12 & 19 & 1111555 \\
\hline & & & & 6 & 12 & 12 & 23 & 1115555 \\
\hline & \multirow{4}{*}{6} & 2 & 12 & 2 & 12 & 4 & 19 & 1111555 \\
\hline & & & & 6 & 12 & 12 & 23 & 1115555 \\
\hline & & 6 & 12 & 2 & 12 & 12 & 23 & 1115555 \\
\hline & & & & 6 & 12 & 12 & 19 & 1111555 \\
\hline \multirow{8}{*}{3} & \multirow{4}{*}{6} & 6 & 12 & 6 & 12 & 12 & 21 & 3333333 \\
\hline & & & & 10 & 20 & 20 & 33 & 3333777 \\
\hline & & 10 & 20 & 6 & 12 & 20 & 33 & 3333777 \\
\hline & & & & 10 & 20 & 20 & 37 & 3337777 \\
\hline & \multirow{4}{*}{10} & 6 & 20 & 6 & 20 & 12 & 33 & 3333777 \\
\hline & & & & 10 & 20 & 20 & 37 & 3337777 \\
\hline & & 10 & 20 & 6 & 20 & 20 & 37 & 3337777 \\
\hline & & & & 10 & 20 & 20 & 33 & 3333777 \\
\hline \multirow{8}{*}{5} & \multirow{4}{*}{6} & 6 & 12 & 6 & 12 & 12 & 23 & 1115555 \\
\hline & & & & 10 & 12 & 12 & 19 & 1111555 \\
\hline & & 10 & 12 & 6 & 12 & 12 & 19 & 1111555 \\
\hline & & & & 10 & 12 & 20 & 23 & 1115555 \\
\hline & \multirow{4}{*}{10} & 6 & 12 & 6 & 12 & 12 & 19 & 1111555 \\
\hline & & & & 10 & 20 & 12 & 23 & 1115555 \\
\hline & & 10 & 20 & 6 & 12 & 12 & 23 & 1115555 \\
\hline & & & & 10 & 20 & 20 & 35 & 5555555 \\
\hline \multirow{8}{*}{7} & \multirow{4}{*}{10} & 10 & 20 & 10 & 20 & 20 & 37 & 3337777 \\
\hline & & & & 14 & 20 & 20 & 33 & 3333777 \\
\hline & & 14 & 20 & 10 & 20 & 20 & 33 & 3333777 \\
\hline & & & & 14 & 20 & 28 & 37 & 3337777 \\
\hline & \multirow{4}{*}{14} & 10 & 20 & 10 & 20 & 20 & 33 & 3333777 \\
\hline & & & & 14 & 28 & 20 & 37 & 3337777 \\
\hline & & 14 & 28 & 10 & 20 & 20 & 37 & 3337777 \\
\hline & & & & 14 & 28 & 28 & 49 & 7777777 \\
\hline
\end{tabular}

Tabela A.2: Representações reduzidas $V_{1}^{3}$ 


\begin{tabular}{|c|c|c|c|c|c|c|c|c|}
\hline$t_{123}$ & $\left|v_{1} \cap v_{2}\right|$ & $\left|v_{1} \cap v_{3}\right|$ & $\left|v_{1}\right|$ & $\left|v_{2} \cap v_{3}\right|$ & $\left|v_{2}\right|$ & $\left|v_{3}\right|$ & grau & tipo \\
\hline \multirow{8}{*}{1} & \multirow{4}{*}{4} & 4 & 8 & 4 & 8 & 8 & 13 & 1111333 \\
\hline & & & & 8 & 16 & 16 & 25 & 1133557 \\
\hline & & 8 & 16 & 4 & 8 & 16 & 25 & 1133557 \\
\hline & & & & 8 & 16 & 16 & 29 & 1135577 \\
\hline & \multirow{4}{*}{8} & 4 & 16 & 4 & 16 & 8 & 25 & 1133557 \\
\hline & & & & 8 & 16 & 16 & 29 & 1135577 \\
\hline & & 8 & 16 & 4 & 16 & 16 & 29 & 1135577 \\
\hline & & & & 8 & 16 & 16 & 25 & 1111777 \\
\hline \multirow{8}{*}{3} & \multirow{4}{*}{4} & 4 & 8 & 4 & 8 & 8 & 15 & 1113333 \\
\hline & & & & 8 & 16 & 16 & 27 & 1133577 \\
\hline & & 8 & 16 & 4 & 8 & 16 & 27 & 1133577 \\
\hline & & & & 8 & 16 & 16 & 31 & 1335577 \\
\hline & \multirow{4}{*}{8} & 4 & 16 & 4 & 16 & 8 & 27 & 1133577 \\
\hline & & & & 8 & 16 & 16 & 31 & 1335577 \\
\hline & & 8 & 16 & 4 & 16 & 16 & 31 & 133557 \\
\hline & & & & 8 & 16 & 16 & 27 & 3333555 \\
\hline \multirow{8}{*}{5} & \multirow{4}{*}{8} & 8 & 16 & 8 & 16 & 16 & 29 & 3335555 \\
\hline & & & & 12 & 16 & 16 & 25 & 1133557 \\
\hline & & 12 & 16 & 8 & 16 & 16 & 25 & 1133557 \\
\hline & & & & 12 & 16 & 24 & 29 & 1135577 \\
\hline & \multirow{4}{*}{12} & 8 & 16 & 8 & 16 & 16 & 25 & 1133557 \\
\hline & & & & 12 & 24 & 16 & 29 & 1135577 \\
\hline & & 12 & 24 & 8 & 24 & 16 & 35 & 1355777 \\
\hline & & & & 12 & 24 & 24 & 41 & 5555777 \\
\hline \multirow{8}{*}{7} & \multirow{4}{*}{8} & 8 & 16 & 8 & 16 & 16 & 31 & 1117777 \\
\hline & & & & 12 & 16 & 16 & 27 & 1133577 \\
\hline & & 12 & 16 & 8 & 16 & 16 & 27 & 1133577 \\
\hline & & & & 12 & 16 & 16 & 25 & 1133557 \\
\hline & \multirow{4}{*}{12} & 8 & 16 & 8 & 16 & 16 & 27 & 1133577 \\
\hline & & & & 12 & 24 & 16 & 31 & 1335577 \\
\hline & & 12 & 24 & 8 & 16 & 16 & 31 & 1335577 \\
\hline & & & & 12 & 24 & 24 & 43 & 5557777 \\
\hline
\end{tabular}

Tabela A.3: Representações reduzidas $V_{2}^{3}$ 


\begin{tabular}{|c|c|c|c|c|c|c|c|c|}
\hline$t_{123}$ & $\mid v_{1} \cap v_{2}$ & $\left|v_{1} \cap v_{3}\right|$ & $\mid v_{1}$ & $\left|v_{2} \cap v_{3}\right|$ & $\mid v_{2}$ & $\left|v_{3}\right|$ & grau & tipo \\
\hline \multirow{8}{*}{1} & \multirow{4}{*}{$1 \quad 2$} & \multirow[t]{2}{*}{2} & \multirow[t]{2}{*}{8} & 2 & 8 & 8 & 19 & 1111555 \\
\hline & & & & 6 & 8 & 8 & 15 & 1111155 \\
\hline & & \multirow[t]{2}{*}{6} & \multirow[t]{2}{*}{8} & 2 & 8 & 8 & 15 & 1111155 \\
\hline & & & & 6 & 8 & 16 & 19 & 1111555 \\
\hline & \multirow{4}{*}{6} & \multirow[t]{2}{*}{2} & \multirow[t]{2}{*}{8} & 2 & 8 & 8 & 15 & 1111155 \\
\hline & & & & 6 & 16 & 8 & 19 & 1111555 \\
\hline & & \multirow[t]{2}{*}{6} & \multirow[t]{2}{*}{16} & 2 & 8 & 8 & 19 & 1111555 \\
\hline & & & & 6 & 16 & 16 & 31 & 1555555 \\
\hline \multirow{8}{*}{3} & \multirow{4}{*}{6} & \multirow[t]{2}{*}{6} & \multirow[t]{2}{*}{16} & 6 & 16 & 16 & 33 & 3333777 \\
\hline & & & & 10 & 16 & 16 & 29 & 3333377 \\
\hline & & \multirow[t]{2}{*}{10} & \multirow[t]{2}{*}{16} & 6 & 16 & 16 & 29 & 3333377 \\
\hline & & & & 10 & 16 & 24 & 33 & 3333777 \\
\hline & \multirow{4}{*}{10} & \multirow[t]{2}{*}{6} & \multirow[t]{2}{*}{16} & 6 & 16 & 16 & 29 & 3333377 \\
\hline & & & & 10 & 24 & 16 & 33 & 3333777 \\
\hline & & \multirow[t]{2}{*}{10} & \multirow[t]{2}{*}{24} & 6 & 16 & 16 & 33 & 3333777 \\
\hline & & & & 10 & 24 & 24 & 45 & 3777777 \\
\hline \multirow{8}{*}{5} & \multirow{4}{*}{6} & \multirow[t]{2}{*}{6} & \multirow[t]{2}{*}{8} & 6 & 8 & 8 & 11 & 1111115 \\
\hline & & & & 10 & 16 & 16 & 23 & 1115555 \\
\hline & & \multirow[t]{2}{*}{10} & \multirow[t]{2}{*}{16} & 6 & 8 & 16 & 23 & 1115555 \\
\hline & & & & 10 & 16 & 16 & 27 & 1155555 \\
\hline & & 6 & 16 & 6 & 16 & 8 & 23 & 1115555 \\
\hline & 10 & & & 10 & 16 & 16 & 27 & 1155555 \\
\hline & & 10 & 16 & 6 & 16 & 16 & 27 & 1155555 \\
\hline & & & & 10 & 16 & 16 & 23 & 1115555 \\
\hline & & 10 & 16 & 10 & 16 & 16 & 25 & 3333337 \\
\hline & 10 & & & 10 & 24 & 24 & 37 & 3337777 \\
\hline & & 14 & 24 & 10 & 16 & 24 & 37 & 3337777 \\
\hline 7 & & & & 10 & 24 & 24 & 41 & 3377777 \\
\hline & & 10 & 24 & 10 & 24 & 16 & 37 & 3337777 \\
\hline & 14 & & & 14 & 24 & 24 & 41 & 3377777 \\
\hline & & 14 & 24 & 10 & 24 & 24 & 41 & 3377777 \\
\hline & & & & 14 & 24 & 24 & 37 & 3337777 \\
\hline
\end{tabular}

Tabela A.4: Representações reduzidas $V_{3}^{3}$ 


\begin{tabular}{|c|c|c|c|c|c|c|c|c|}
\hline$t_{123}$ & $\left|v_{1} \cap v_{2}\right|$ & $\left|v_{1} \cap v_{3}\right|$ & $\left|v_{1}\right|$ & $\left|v_{2} \cap v_{3}\right|$ & $\left|v_{2}\right|$ & $\left|v_{3}\right|$ & grau & tipo \\
\hline \multirow{8}{*}{1} & \multirow{4}{*}{4} & \multirow[t]{2}{*}{4} & \multirow[t]{2}{*}{12} & 4 & 12 & 8 & 21 & 1133355 \\
\hline & & & & 8 & 12 & 16 & 25 & 1133557 \\
\hline & & \multirow[t]{2}{*}{8} & \multirow[t]{2}{*}{12} & 4 & 12 & 16 & 25 & 1133557 \\
\hline & & & & 8 & 12 & 16 & 21 & 1111377 \\
\hline & \multirow{4}{*}{8} & \multirow[t]{2}{*}{4} & \multirow[t]{2}{*}{12} & 4 & 12 & 8 & 17 & 1111337 \\
\hline & & & & 8 & 20 & 16 & 29 & 1135577 \\
\hline & & \multirow[t]{2}{*}{8} & \multirow[t]{2}{*}{20} & 4 & 12 & 16 & 29 & 1135577 \\
\hline & & & & 8 & 20 & 16 & 33 & 1155777 \\
\hline \multirow{8}{*}{3} & \multirow{4}{*}{4} & \multirow[t]{2}{*}{4} & \multirow[t]{2}{*}{12} & 4 & 12 & 8 & 23 & 1113377 \\
\hline & & & & 8 & 12 & 16 & 27 & 1133577 \\
\hline & & \multirow[t]{2}{*}{8} & \multirow[t]{2}{*}{12} & 4 & 12 & 16 & 27 & 1133577 \\
\hline & & & & 8 & 12 & 16 & 23 & 1333355 \\
\hline & \multirow{4}{*}{8} & \multirow[t]{2}{*}{4} & \multirow[t]{2}{*}{12} & 4 & 12 & 8 & 19 & 1133335 \\
\hline & & & & 8 & 20 & 16 & 31 & 1335577 \\
\hline & & \multirow[t]{2}{*}{8} & \multirow[t]{2}{*}{20} & 4 & 12 & 16 & 31 & 1335577 \\
\hline & & & & 8 & 20 & 16 & 35 & 3355577 \\
\hline \multirow{8}{*}{5} & \multirow{4}{*}{8} & \multirow[t]{2}{*}{8} & \multirow[t]{2}{*}{12} & 8 & 12 & 16 & 21 & 1133355 \\
\hline & & & & 12 & 20 & 16 & 25 & 1133557 \\
\hline & & \multirow[t]{2}{*}{12} & \multirow[t]{2}{*}{20} & 8 & 12 & 16 & 25 & 1133557 \\
\hline & & & & 12 & 20 & 24 & 37 & 3555577 \\
\hline & & 8 & 20 & 8 & 20 & 16 & 33 & 3355557 \\
\hline & 12 & & & 12 & 20 & 16 & 29 & 1135577 \\
\hline & & 12 & 20 & 8 & 20 & 16 & 29 & 1135577 \\
\hline & & & & 12 & 20 & 24 & 33 & 1155777 \\
\hline & & 8 & 12 & 8 & 12 & 16 & 23 & 1113377 \\
\hline & 8 & & & 12 & 20 & 16 & 27 & 1133577 \\
\hline & & 12 & 20 & 8 & 12 & 16 & 27 & 1133577 \\
\hline 7 & & & & 12 & 20 & 24 & 39 & 1557777 \\
\hline & & 8 & 20 & 8 & 20 & 16 & 35 & 1157777 \\
\hline & 12 & & & 12 & 20 & 16 & 31 & 1335577 \\
\hline & & 12 & 20 & 8 & 20 & 16 & 31 & 1335577 \\
\hline & & & & 12 & 20 & 24 & 35 & 3355577 \\
\hline
\end{tabular}

Tabela A.5: Representações reduzidas $V_{4}^{3}$ 


\begin{tabular}{|c|c|c|c|c|c|c|c|c|}
\hline$t_{123}$ & $\left|v_{1} \cap v_{2}\right|$ & $\left|v_{1} \cap v_{3}\right|$ & $\left|v_{1}\right|$ & $\left|v_{2} \cap v_{3}\right|$ & $\left|v_{2}\right|$ & $\left|v_{3}\right|$ & grau & tipo \\
\hline \multirow{8}{*}{1} & \multirow{4}{*}{4} & \multirow[t]{2}{*}{4} & \multirow[t]{2}{*}{12} & 4 & 8 & 8 & 17 & 1113335 \\
\hline & & & & 8 & 16 & 16 & 29 & 1335557 \\
\hline & & \multirow[t]{2}{*}{8} & \multirow[t]{2}{*}{12} & 4 & 8 & 16 & 21 & 1113357 \\
\hline & & & & 8 & 16 & 16 & 25 & 1113577 \\
\hline & \multirow{4}{*}{8} & \multirow[t]{2}{*}{4} & \multirow[t]{2}{*}{12} & 4 & 16 & 8 & 21 & 1113357 \\
\hline & & & & 8 & 16 & 16 & 25 & 1113577 \\
\hline & & \multirow[t]{2}{*}{8} & \multirow[t]{2}{*}{20} & 4 & 16 & 16 & 33 & 1355577 \\
\hline & & & & 8 & 16 & 16 & 29 & 1115777 \\
\hline \multirow{8}{*}{3} & \multirow{4}{*}{4} & \multirow[t]{2}{*}{4} & \multirow[t]{2}{*}{12} & 4 & 8 & 8 & 19 & 1113337 \\
\hline & & & & 8 & 16 & 16 & 31 & 1135777 \\
\hline & & \multirow[t]{2}{*}{8} & \multirow[t]{2}{*}{12} & 4 & 8 & 16 & 23 & 1133357 \\
\hline & & & & 8 & 16 & 16 & 27 & 1333557 \\
\hline & \multirow{4}{*}{8} & \multirow[t]{2}{*}{4} & \multirow[t]{2}{*}{12} & 4 & 16 & 8 & 23 & 1133357 \\
\hline & & & & 8 & 16 & 16 & 27 & 1333557 \\
\hline & & \multirow[t]{2}{*}{8} & \multirow[t]{2}{*}{20} & 4 & 16 & 16 & 35 & 1355777 \\
\hline & & & & 8 & 16 & 16 & 31 & 3335557 \\
\hline \multirow{8}{*}{5} & \multirow{4}{*}{8} & \multirow[t]{2}{*}{8} & \multirow[t]{2}{*}{12} & 8 & 16 & 16 & 25 & 1333555 \\
\hline & & & & 12 & 16 & 16 & 21 & 1113357 \\
\hline & & \multirow[t]{2}{*}{12} & \multirow[t]{2}{*}{20} & 8 & 16 & 16 & 29 & 1335557 \\
\hline & & & & 12 & 16 & 24 & 33 & 1355577 \\
\hline & & 8 & 20 & 8 & 16 & 16 & 29 & 1335557 \\
\hline & 12 & & & 12 & 24 & 16 & 33 & 1355577 \\
\hline & & 12 & 20 & 8 & 16 & 16 & 25 & 1113577 \\
\hline & & & & 12 & 24 & 24 & 37 & 1555777 \\
\hline & & 8 & 12 & 8 & 16 & 16 & 27 & 1113777 \\
\hline & 8 & & & 12 & 16 & 16 & 23 & 1133357 \\
\hline & & 12 & 20 & 8 & 16 & 16 & 31 & 1135777 \\
\hline 7 & & & & 12 & 16 & 24 & 35 & 1355777 \\
\hline & & 8 & 20 & 8 & 16 & 16 & 31 & 1135777 \\
\hline & 12 & & & 12 & 24 & 16 & 35 & 1355777 \\
\hline & & 12 & 20 & 8 & 16 & 16 & 27 & 1333557 \\
\hline & & & & 12 & 24 & 24 & 39 & 3555777 \\
\hline
\end{tabular}

Tabela A.6: Representações reduzidas $V_{5}^{3}$ 


\section{Referências Bibliográficas}

[1] R.L. Griess Jr., Code loops, J.Algebra 100 (1986) 224-234.

[2] R.H. Bruck, A survey of binary systems, Springer-Verlag, Berlin, 1958.

[3] O. Chein and E.G. Goodaire, Moufang Loops with a Unique Nonidentity Commutator (Associator, Square), J. Algebra 130 (1990) 369-384.

[4] H.O. Pflugfelder, Quasigroups and Loops: An Introduction, Berlin, 1990.

[5] O. Chein, H.O. Pflugfelder, and J.D.H. Smith, Quasigroups and Loops: Theory and Applications, Berlin, 1990.

[6] E.G. Goodaire, E. Jaspers and C. Polcino, Alternative Loop Rings, North Holland Math, Studies N.184, Elsevier, Amsterdam, 1996.

[7] J. Conway, A simple construction of the Fischer-Griess monster, Invent. Math. 79 (1985) 513-540.

[8] O. Chein and E.G. Goodaire, Isomorphism of loops which have alternative loops rings, Comm. Algebra 13 (1985) 1-20.

[9] O. Chein and E.G. Goodaire, Loops whose loop rings over rings of characteristic two are alternative, Comm. Algebra, in press.

[10] E. G. Goodaire, Alternative loop rings, Publ. Math. Debrecen 30 (1983) 31-38. 
[11] O. Chein and E.G. Goodaire, Code Loops are RA2 Loops, J. Algebra 130, (1990) $385-387$.

[12] A. Garcia, Y. Lequain, Elementos de Álgebra, 5.ed. Rio de Janeiro:IMPA, 2008. 